\title{
Ventricular impulse formation and the influence of digitalis intoxication
}

Citation for published version (APA):

Gorgels, A. P. M. (1985). Ventricular impulse formation and the influence of digitalis intoxication. [Doctoral Thesis, Maastricht University]. Rijksuniversiteit Limburg. https://doi.org/10.26481/dis.19850628ag

Document status and date:

Published: 01/01/1985

DOI:

10.26481/dis.19850628ag

Document Version:

Publisher's PDF, also known as Version of record

\section{Please check the document version of this publication:}

- A submitted manuscript is the version of the article upon submission and before peer-review. There can be important differences between the submitted version and the official published version of record.

People interested in the research are advised to contact the author for the final version of the publication, or visit the DOI to the publisher's website.

- The final author version and the galley proof are versions of the publication after peer review.

- The final published version features the final layout of the paper including the volume, issue and page numbers.

Link to publication

\footnotetext{
General rights rights.

- You may freely distribute the URL identifying the publication in the public portal. please follow below link for the End User Agreement:

www.umlib.nl/taverne-license

Take down policy

If you believe that this document breaches copyright please contact us at:

repository@maastrichtuniversity.nl

providing details and we will investigate your claim.
}

Copyright and moral rights for the publications made accessible in the public portal are retained by the authors and/or other copyright owners and it is a condition of accessing publications that users recognise and abide by the legal requirements associated with these

- Users may download and print one copy of any publication from the public portal for the purpose of private study or research.

- You may not further distribute the material or use it for any profit-making activity or commercial gain

If the publication is distributed under the terms of Article $25 \mathrm{fa}$ of the Dutch Copyright Act, indicated by the "Taverne" license above, 


\section{Ventricular impulse formation and the influence of digitalis intoxication.}

\section{Proefschrift}

Ter verkrijging van de graad van doctor in de Geneeskunde aan de Rijksuniversiteit Limburg te Maastricht, op gezag van de Rector Magnificus Prof. Dr. F.I.M. Bonke, volgens besluit van het College van Dekanen in het openbaar te verdedigen in de aula van de universiteit op 28 juni 1985 des namiddags te vier uur precies.

door

Antonius Peter Maria Gorgels geboren te Kerkrade. 
Promotor : Prof. Dr. H.J.J. Wellens

Referenten : Prof.Dr.M.A.Allessie

Prof. Dr. M.R. Rosen

Het verschinnen wan dit proefschrift werd mogelijk gemaakı door steun van de stichting RESCAR (stichting ter bevordering van research in de cardiologie), Maastricht, en de Nederlandse Hartstichting

Knoll, B.V.

Sandoz, B.V.

Welcome, B.V. 
"Diss gewechss würdr von unsern Teutschen fingerhut geheyssen, darumb das seine blumen einen fingerhut, so man zu dem näen braucht, gantz und gar ähnlich seind. Man mags in mittler zeit, bis man einen bessern namen findt, wie wir in unserem Lateinischen Kreuterbuch gethan haben, Digitalem zu Latein, derm Teutschen namen nach nennen." Leonhard Fuchs 1542 (from ref. 45).

To the memory of my father.

To my mother.

To Janneke, Tönnes, Christoffel and Hannes. 
84 HISTOIRE DE L'ACADEMIEROYALE

\section{OBSERVATION DE BOTANIQUE.}

A Salerne, Médecin à Orléans \& Correfpondant de 1 1. l'Académie, ayant appris que plufieurs dindonneaux étoient morts pour avoir mangé des feuilles de la grande digitale'à fleurs rouges, qu'on lẹur avoit données par hafard pour du bouillon-blane, voulut s'affurer de ce qui en étoit; il donna pour çela de ces mêmes feuilles à un gros dindon : quoique cet animal fût fort \& vigoureux, que la plante eût peu de vertu, tant parce que les feuilles étoient cueillies depuis fept à huit jours, que parce que l'expérience avoit été faite en hyver, \& qu'il n'en eût mangé qu'une feule fois, il en fut cependant fi malade, qu'il ne pouvoit fe tenir fur fes jambes; il paroiffoit ivre, \& rendoit des excrémeñs rougeatres; huit jours de bonne nourriture fuffirent à peine pour le rétablir. M. Salerne jugea à propos de faire une feconde expérience, $\&$ de la pouffer plus loin; il donna, au mois de Décembre, des feuilles de la même plante hachées, mêlées avec du fon de froment, à un coq d'Inde vigoureux, \& qui pefoit fept livres: dès qu'il en eut mangé, il parut trifte $8 x$ mélancolique; fes plumes étoient hériffées, \& fon col pale \& retiré; il en mangea cependant encore quatre jours, pendant lefquels il en confuma environ une demi-poignée, qui avoient été cueillies depuis environ huit jours, \& comme nous l'avons dit, dans une faifon très-avancée. Dès le premier repas, on remarqua que les excrémens, naturellement verds \& bien liés, étoient devenus rougeâtres \& liquides, comme s'il étété attaqué de la dyfenterie. L'animal ne voulant plus abfolument manger de cette patée qui lui avoit été fi nuifible, on fut obligé de lui donner du fon délayé avec de l'eau, mais çependant il continua d'être trifte $\&$ dégoûté ; il lui prenoit de temps en temps des convulfions fi vives, qu'il fe laiffoit tomber ; lorfqu'il s'étoit relevé, il marchoit 
D E S S C I E N C E S.

comme s'il eût été ivre ; $\&$ quoiqu'il eût de quoi fe percher, il fe tenoit toujours à terre; il pouffoit prefque fans ceffe des cris plaintifs; il refuloit tous les alimens, même l'orge \& l'avoine, dont on fait que ces animaux font très-friands : au bout de cinq ou fix jours, les excrémens devinrent blancs comme de la chaux nouvellement éteinte, puis jaunes, verdâtres $\&$ noirâtres. Enfin, le dix-huitième jour de l'expérience, il mourut dans une maigreur fi grande, que de fept livres qu'il pefoit avant quon la commençât, 1 l étoit réduit à trois : on louvrit, $\&$ on trouva le cœur, le poumon, le fore $\&$ la véficule du fiel flérris ; l'eftomac avoit fon velouté, mais il étoit abfolument vuide. Au moment qu'on l'ouvrit, il rendit par le bec \& par l'anus une matière verte $\&$ liquide , femblable $\alpha$ de la lie d'huile d'olives; certe matière étoit plus épaiffe dans le géfier \& dans les inteftins. On voit par ces expériences le dérangement que l'ufage de cetce plante peut caufer dans les organes de ces animaux, \& combien on doit être attentif à la détruire dans les endroits où on les élève.

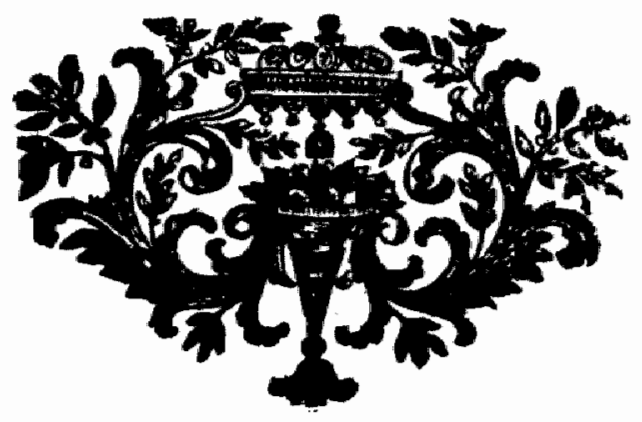




\section{F. Salerne}

Obserwation de Botanique. Mist. Acad. Roy. Sci.

Paris, Année 1748 (1752); 74-75

(Primed with permission from the Biblioheque Nationale, Paris, France) 
Table of contents.

Salerne F. Observation de Botanique.

Abbreviations.

Chapter 1 Introduction.

Chapter 2 Impulse formation in cardiac tissues.

Chapter 3 Extrastimulus related shortening of the first postpacing interval in digitalis induced ventricular tachycardia.

Chapter 4 Effect of programmed electrical stimulation on the first postpacing QRS-interval during digoxin intoxication.

Chapter 5 Effect of different modes of stimulation on the configuration of the first QRS-complex following pacing during digitalis-induced ventricular tachycardia.

Chapter 6 Effect of overdrive stimulation on the normal idioventricular rhythm.

Chapter 7 Effect of lidocaine, verapamil, isoprenaline and ouabain on ventricular impulse formation.

Summary.

Samenvatting.

References.

Acknowledgements.

Curriculum vitae. 
ABBREVIATIONS.

$\begin{array}{ll}V_{\mathrm{at}} & : \text { the spontaneous QRS-complex before stimulation. } \\ \mathrm{V}_{\mathrm{s}} & : \text { the stimulated QRS-complex. } \\ \mathrm{V}_{\mathrm{s} 1} & : \text { the QRS-complex induced by an extrastimulus. } \\ \mathrm{V} & : \text { the first postpacing QRS-complex. } \\ \mathrm{V}_{2} & : \text { the second postpacing QRS-complex. } \\ \mathrm{s} & : \text { timulus artefact. } \\ \mathrm{n}\left(\mathrm{V}_{\mathrm{s}}\right) & \text { number of stimuli. } \\ \mathrm{V}_{\mathrm{s}}-\mathrm{V}_{\mathrm{al}} & : \text { the last prepacing interval. } \\ \mathrm{V}_{\mathrm{s}}-\mathrm{V}_{\mathrm{s}} & : \text { the interstimulus interval. } \\ \mathrm{V}_{\mathrm{s}}-\mathrm{V} & : \text { the first postpacing interval. } \\ \mathrm{V}_{\mathrm{s} 1}-\mathrm{V} & : \text { the first postpacing interval following an extrastimulus. } \\ \mathrm{V}-\mathrm{V}_{2} & : \text { the second postpacing interval. }\end{array}$




\section{Chapter 1}

\section{Introduction.}

This work deals with the effect of programmed electrical stimulation of the heart on ventricular thythm in conscious dogs with chronic complete atrioventricular block with and without digitalis intoxication.

The study was initiated for the following reasons.

An arrhythmia in a patient using digitalis frequently confronts the clinician with the possibility of digitalis intoxication. It may however often be difficult to proof that this diagnosis indeed is the cause of the arrhythmia.

Better understanding of cellular mechanisms leading to digitalis induced arrhythmias has been obtained during recent years using programmed electrical stimulation.

Application of this technique in dogs with digitalis intoxication could be of help for the understanding of the mechanisms of digitalis-induced arrhythmias in the intact heart. It may also be a clinically useful technique for diagnosing digitalis intoxication.

\section{The clinical problem of digitalis intoxication}

Digitalis derivatives have been used since centuries for improvement of cardiac contractility. Also today digitalis glycosides are used frequently because these drugs are suitable for chronic oral use to increase the contractility of the heart without increasing the heart rate ${ }^{45}$. In the Netherlands $2 \%$ of the population are regular users of these drugs 118,140 . Unfortunately the therapeutic range is narrow what frequently leads to cardiac and extracardiac signs of toxicity ${ }^{113}$. Recent estimations suggest a $5-15 \%$ incidence of digitalis intoxication in patients at the moment of hospital admission ${ }^{110}$.

During the last decades much effort has been spent to reduce the occurrence of digitalis intoxication. Bioawailability of orally ingested digoxin has been standardized, after it was recognized that marked differences in serum digoxin levels were present between different brands of digoxin tablets ${ }^{79,83}$ and even between batches of tablets from the same manufacturer ${ }^{53}$. Reducing variations in dissolution rates by standardizing particle size and improved mixing during tabletting has led to a more constant bioavailability of digoxin tablets ${ }^{107,111}$.

Since the development of immunologic methods for determination of plasma levels of digoxin ${ }^{11,108}$ and digitoxin ${ }^{91}$, radio-immunoassay has become widely available to help the digitalization of the individual patient.

However, determination of plasma levels of digitallis derivatives has been found to be of limited value because of the frequent overlap in plasma levels of patients with and without evidence of toxicity ${ }^{85,109}$

Caution has been advised therefore against uncritical acceptance of the value of digitalis plasma level as guide during digitalis therapy $46,85,118,140$.

A high incidence of digitalis toxicity occurs because many factors influence resorption, metabolism and excretion of digitalis $46,85,118,140$.

Alterations in ionic conditions and acid-base abnormalities (hypokalemia, hypomagnesemia, hypercalcemia, hyponatremia, alkalosis) decrease the tolerance to 
these drugs. Other factors are hypoxemia, stroke, impaired renal function, hypothyroidism, myocardial disease, patient age, and the concomitant use of drugs like quinidine and L-dopa.

This has led to advocation of adapted dose regimens taking some of these factors into account ${ }^{65,90 \%}$.

\section{Digitalis induced cardiac arrhythmias}

The toxic manifestations of digitalis derivatives on the cardiac rhythm are characterized by enhanced impulse formation and depressed impulse conduction in different sites of the heart $18,140$. Depressed impulse conduction leads clinically to different degrees of block within the sinus node and atrioventricular node. Enhanced impulse formation occurs in the atria, the atrioventricular node and in the ventricles. Because enhanced impulse formation and depressed impulse conduction occur simultaneously, complex arrhythmias are frequently abserved ${ }^{118,140}$.

During the last decades the underlying mechanisms of digitalis action have been studied extensively. One of the most important findings was that digitalis glycosides act through inhibition of the sodium-potassium pumping mechanism ${ }^{45,112}$. It was also found that, based upon this inhibition, triggered activity, induced by delayed afterdepolarizations, can occur, leading to impulse formation in isolated Purkinje fibers ${ }^{17,32,48,57,97}$.

\section{Outline of the study}

Because of its obvious diagnostic and therapeutic implications, we wanted to know whether triggered activity also plays a role during digitalis intoxication in the intact heart. As the behaviour of delayed afterdepolarizations has been studied by programmed electrical stimulation, this method was also used in our study of digitalis intoxication (chapters 3-5). Since it was not known whether the findings were specific for digitalis toxicity, or were, at least in part related to the pacing modes, experiments were also performed without digitalis intoxication using similar pacing protocols (chapter 6). To further evaluate the results obtained during these control experiments, a study was done using programmed electrical stimulation combined with drugs specifically influencing cellular mechanisms of impulse formation (chapter 7 ). 


\section{Chapter 2}

\section{Impulse formation in cardiac tissues.}

This chapter deals briefly with the most important mechanisms involved in normal and abnormal impulse formation in cardiac tissues. For this purpose, we will restrict ourselves to aspects of impulse formation which relate to the subject of our investigation. More detailed information is given in a number of recently published articles $9,18,35,37,40,54,55$, $56,85,98,100,101,102,127,129,131,146,152,155,157$

Normally the sinoatrial node dominates the rhythmic activity of the heart. The cardiac impulse spreads through the atria, the atriowentricular node, the ventricular conducting system and the ventricles, until the entire heart is activated. This sequence of events is repeated 60 to 100 times each minute. Impulse conduction can occur because all cardiac cells are excitable: they respond to a stimulus which is sufficiently strong to bring them to threshold by generating an action potential ${ }^{102}$. Certain cardiac fibers are automatic as well; they can depolarize and spontaneously initiate an action potential. When sinus node function stops, specialized fibers in the atrium, the atrioventricular junction and the ventricular specialized conducting system can depolarize spontaneously and function as automatic pacemakers ${ }^{102}$. The spontaneous activity of these cells results from the fact that each action potential is followed by a spontaneous slow depolarization, that brings the membrane to its threshold potential, thereby evoking an action potential ${ }^{18}$.

\section{Normal impulse formation}

Cells for impulse generation can be subdivided into two main classes ${ }^{55}$ : cells which generate fast responses and cells generating slow repsonses. Fast responses are found in specialized fibers of the atria and the His-Purkinje system. Cells, normally generating slow responses, are the primary pacemaker cells of the sinus node, the cells of the $\mathrm{N}$-region of the atrioventricular node and perhaps cells in the mitral and tricuspid valves ${ }^{55}$.

\section{The fast response}

The action potential of the fast responses is generally divided in four phases ${ }^{102}$ (figure 1). In Purkinje fibers depolarization of the cell membrane during phase 0 occurs by a voltage change from -90 to $+30 \mathrm{mv}$. Depolarization is carried by a sodium inward curremt through fast channels. During phase 1 partial repolarization is induced by inactivation of the fast inward current and activation of an outward potassium current. Subsequently a slow inward current is activated which is carried by calcium and sodium (phase 2). With time the slow inward channel closes partially and another potassium channel opens. The net current becomes outward and the membrane potential shifts to the resting value (phase 3) ${ }^{102}$. In Purkinje fibers the transmembrane diastolic potential (phase 4) is approximately $-90 \mathrm{mV}$. This potential is the result of a concentration gradient for potassium across the cell membrane, such that the ratio of intracellular to extracellular potassium gradient is about 30:1. The transmembrane potassium gradient is established by the enzyme $\mathrm{Na}^{+}-\mathrm{K}^{+}$ATPase, which pumps sodium out and potassium into the cell. This transport of sodium and potassium is electrogenic in nature, more sodium being 
pumped out than potassium being pumped in. The resulting outward current contributes to the resting potential and influences the time course of the action potential and phase 4 depolarization ${ }^{102}$.

The mechanism of this diastolic depolarization is a matter of controversy. Previously it was thought that depolarization occurred through a potassium outward current, that activated after return to maximal diastolic potential and then gradually diminished ${ }^{129}$. Recent data suggest however that the pacemaker current is mainly carried by an increasing sodium inward current ${ }^{26}$.

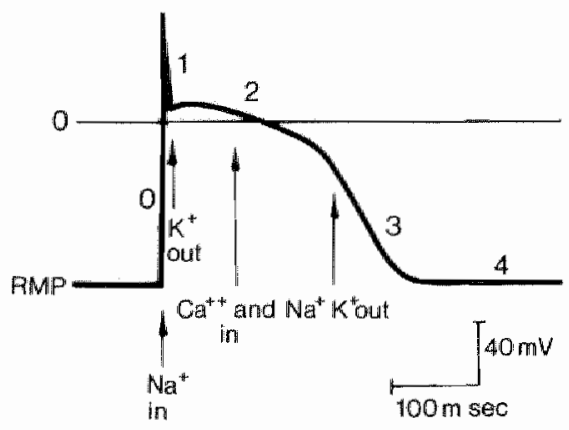

Figure 1

Transmembrane action potential of a Purkinje fiber; $R M P=$ resting membrane potential. The major ionic: currents responsible for the action potential during phases 0 (rapid usptroke), 1 initial repolarization), 2 (plateau), and 3 (repolarization) are indicated. For futher description of these and of phase 4, see text (from ref. 102, printed with permission from F.A. Davis Co).

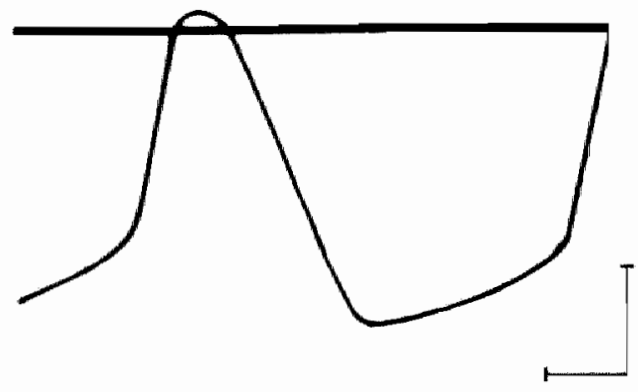

Figure 2

Transmembrane potential of rabbit sinus node fiber. Note low resting potential as compared to that of Purkinje fiber in figure 1 (vertical calibration, $20 \mathrm{mv}$; , horizontal, $100 \mathrm{mnsec}$ ) (from ref. 102, printed with permission from Martinus Nijhoff Publishers).

\section{The slow response}

Cells, exhibiting slow responses, have low resting membrane potentials ( -60 mv to -70 $\mathrm{mv}$ ) and slowly rising action potentials ${ }^{102}$ (figure 2). In nodal cells depolarization is carried by a current through slow inward channels. Because the resting membrane potential is low, the fast channels are largely inactivated. Phase 4 depolarization results from an inward current that is activated on repolarization ${ }^{37}$. 
Conduction velocity is faster in cells exhibiting fast as compared to those having slow responses ${ }^{55}$. In diseased hearts slow responses may occur in other sites of the heart than the sinus node and the atrioventricular node. They may provide the prerequisite for areas of slow conduction leading to the occurrence of reentry ${ }^{55}$.

\section{The hierarchy between normal cardiac pacemakers}

As pointed out above, the sinus node usually dominates cardiac rhy thm. Other potentially impulse generating foci usually only come into play under abnormal circumstances like conduction block, or because of acceleration of these pacemakers above the sinus rate. It is however known already for a long time ${ }^{127}$ that when the dominant cardiac pacemaker suddenly stops, usually a period of quiescence is observed before a subsidiary pacemaker begins to discharge. This phenomenon is called overdrive suppression.

Overdrive suppression of ventricular pacemakers has been studied extensively. It was found that the amount of overdrive suppression was related to the rate and duration of the preceding rhythm, be it spontaneous or induced by electricall stimulation ${ }^{127}$. In contrast to the sinus node, overdrive suppression appeared not to be mediated by increased vagal activity. Also distention of the ventricles during the arrest or a reflex release of catecholamines, due to a fall in blood pressure, have not been found to play a role ${ }^{127}$. Ventricular overdrive pacing leads to an initial increase of the extracellular potassium concentration and a decrease of the diastolic membrane potential. The possibility has been studied whether this increase in extracellular potassium concentration played an important role in overdrive suppression of ventricular pacemakers. It was found that this potassium loss was transient and disappeared after longer drives whereas the amount of overdrive suppression increased.

Probably the most important factor for the occurrence of overdrive suppression in ventricular tissue is activation of the sodium-potassium pump ${ }^{129}$. As pointed out before, this mechanism causes hyperpolarization of the resting membrane potential because of its electrogenicity. The responsible enzyme is activated by an increase in intracellular sodium and/or extracellular potassium concentration ${ }^{40}$.

As a consequence of overdriving the ventricle or Purkinje fibers, more sodium enters the cell because of more action potentials per unit time. When overdrive stops the pump does not cease its activity abruptly and therefore keeps the diastolic depolarization negative to threshold.

The possible role of an increase of the internal calcium concentration should also be considered. Internal calcium accumulation occurs during rapid overdrive pacing. It has been found that an increased calcium concentration increases potassium conductance ${ }^{89}$. When a deactivation of an outward potassium current plays a role in the diastolic depolarization, it is conceivable that this deactivation is inhibited, leading to prolongation of the diastolic depolarization ${ }^{89}$.

\section{Arrhythmias}

According to the classification of Hoffman and Cranefield ${ }^{56}$, cardiac arrhythmias are based on three mechanisms:

1. Abnormal impulse generation, including normal and abnormal automaticity and triggered activity. 
2. Abnormal impulse conduction, including reentry.

3. Simultaneous abrormalities of impulse generation and conduction, including parasystole.

\section{Reentry}

Probably the best studied mechanism of cardiac arrhytmias both in the animal and the human heart is reentry. Reentry, or circus movement of excitation, occurs when an impulse that has excited the heart does not die out, but finds a pathway of excitable fibers over which it may return to reexcite part or all of the heart ${ }^{155}$.

For reentry to occur the following requirements have to be fulfilled $\left.{ }^{155}: 1\right)$ Cardiac tissue has to be or has to be brought in a state of inhomogeneity i.e. differences must exist in refractoriness and impulse conduction. 2) This must result in unidirectional block in one pathway in the cardiac tissue and slowing of impulse conduction in another pathway. 3) This enables the impulse to reenter in the former pathway leading to reexcitation of the heart ${ }^{147,155}$ (figure 3). 4) Another requirement is that the wave length (conduction velocity $\mathrm{x}$ refractory period) must be shorter than the length of the circuit to allow the tissue into which the impulse is reentering to recover excitability ${ }^{155}$. This means that the revolution time in the circuit has to be longer than the longest refractory period of the components of the circuit.
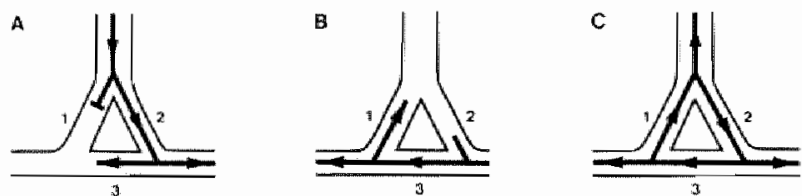

Figure 3

The mechanism of re-entry. Panel A shows arrival of an impulse at the site of division into two pathways. Because of differences in the duration of the refractory period of pathway 1 and 2 the impulse is blocked in pathway 1 and exclusively conducted over pathway 2. Panel B shows how the impulse coming from pathwa 2 after being conducted over pathway 3 re-enters the distal portion of pathway 1 . If the proximal portion of pathwily I has recovered, the impulse is able to be conducted back to the site of origin. If this mechanism perpetuates (panel $C$ ), a regular re-entry tachycardia results. (from ref. 147 , printed with permission from Véwé publishen'si,

Reentry may occur through well defined pathways like distal ends of the Purkinje fiber system, the bundle branch system, surviving muscle fibers in healed infarcts or accessory atrioventricular connections ${ }^{155}$. The latter provides, as emphasized by Wellens, an elegant model for studying reentry in the human heart ${ }^{138,146}$. On the other hand reentry can occur in the absence of an anatomic obstacle. This kind of reentry is known as the leading circle mechanism, described by Allessie et al ${ }^{\prime}$. In this model the length of the circuit is defined by the conduction velocity and refractory period of the tissue. Reentry can also be divided in ordered and random reentry. In ordered reentry, the reentry circuit is fixed, whereas in random reentry activation fronts travel along continuously changing pathways through the cardiac tissue ${ }^{155}$. The latter mechanism is active in atrial and ventricular fibrillation. This multiple wavelet theory as proposed by Moe for atrial fibrillation ${ }^{88}$ has been confirmed experimentally by Allessie et al ${ }^{3}$. 
Also, reentry not only occurs as a circus movement of electrical activation, but also as reflection of the impulse in tubelike structures as described by Wit er al ${ }^{1+49}$. Antzelevitch et al ${ }^{5}$ gave an alternative explanation for reflected responses in Purkinje fiber bundles. They showed that reflection could occur through electrotronic transmission of an impulse through an inexcitable segment.

Reentry has been found to occur in different sites both in the animal and the human heart, including the sinus node ${ }^{2,145}$ the atria ${ }^{4,65}$, the atrioventricular node ${ }^{10,63}$ the ventricles 135,154 and over accessory atrioventricular connections ${ }^{28,138,141,146}$.

Different pathological conditions may lead to reentry as ischemia ${ }^{64}$, myocardial infarction $30,136,156$, right wentricular dysplasia, cardiomyopathies, valvular heart disease, and congenital heart disease ${ }^{69}$. Also no demonstrable structural abnormalities may be present. Reentry can be induced and terminated by appropriately timed premature electrical stimuli ${ }^{146}$.

As Wellens has pointed out, the ability to terminate a reentry circuit is related to 1) the distance between the reentry circuit and the site of stimulation 2) the duration of the refractory period of the tissue at the site of stimulation 3) the conduction velocity of the tissue in between the site of stimulation and the site of the reentry circuit 4) the excitable gap within the reentry circuit ${ }^{146}$.

Since the technique of programmed electrical stimulation for the study of supraventricular arrhythmias was introduced in man by Durrer et al ${ }^{27}$ and Coumel et al ${ }^{16}$ in 1967 and for studying ventricular tachycardia in 1972 by Wellens et al ${ }^{135}$, it has opened new ways for the pharmacological ${ }^{84,137,139,143}$, electrical ${ }^{24,25,144}$ and surgical treatment ${ }^{69,148}$ of many clinically occurring arrhythmias.

\section{Triggered activity}

\section{Introduction}

The term triggered activity, initially introduced by Cranefield and Aronson ${ }^{18}$, is used for arrhythmias which are caused by afterdepolarizations. Afterdepolarizations are transient depolarizations of the membrame potential during or after an action potential and are caused by this action potential ${ }^{18}$. This means that they do not occur spontaneously. When the amplitude of an afterdepolarization is sufficiently high, threshold can be attained and a normal action potential develops ${ }^{18}$. This action potential can again induce an afterpotential, resulting in self sustaining rhythmic activity ${ }^{18}$.

Triggered activity has gained much attention during the last decade, but the possible existence was signaled already earlier in this century. A description pointing to a mechanism like triggered activity can be found in an article by Rothberger and Winterberg as early as $1911^{103}$. During studies on the influence of strophantidin on impulse formation in the heart, these investigators noticed that during sympathetic stimulation sinus beats were followed by ventricular extrasystoles, having a coupling interval similar to the preceding sinus cycle length. They accurately described the dependency of ventricular ectopy from the presence of digitalis and from acceleration of the preceding rate by sympathetic stimulation. To explain the underlying mechanism of this finding the authors referred to the Engelmann-Wenckebach hypothesis, which implied that ventricular foci may become automatic through preceding activation by supraventricular beats. Unfortunately, they gave no further reference to the original 
description by Engelmann and Wenckebach. Later, similar observations were done by Vassalle ${ }^{120}$ in the digitalis intoxicated intact dog heart. As reviewed by Cranefield ${ }^{18}$, Segers (1941) ${ }^{106}$ and Bozler (1943) had previously shown the existence of early and delayed afterdepolarizations and their ability to induce extrasystoles. They also observed that afterdepolarizations became enhanced by elevated calcium concentrations and drugs like aconitine, veratrine, adrenaline, strophantidin and digitalis. Depression of afterdepolarizations was observed in the presence of elevated potassium concentrations and acetylcholine.

Whereas the studies of Segers and Bozler relied on records of monophasic action potentials, Trautwein et al were the first to use intracellular recordings for the study of afterdepolarizations ${ }^{116}$.

\section{Early and delayed afterdepolarizations}

Afterdepolarizations can occur during or after the repolarization phase of the action potential. The former are called early afterdepolarizations and the latter delayed afterdepolarizations ${ }^{18}$. Early afterdepolarizations are depolarizing potentials occurring during phase 2 or phase 3 of repolarization (figure 4). During phase 2 early afterdepolarizations do not induce triggered activity, in contrast to early afterdepolarizations occurring during phase 3 . Early afterdepolarizations can occur when besides the normal resting potential another stable resting potential occurs at a less negative value ${ }^{37}$. They have been induced in isolated cardiac tissues under a variety of conditions that increase inward current or reduce repolarizing current (catecholamines, reduced potassium concentrations, reduced $\mathrm{pH}$, low calcium concentrations, hypoxia, aconitine, $\mathrm{N}$-acetyl procaïnamide, sotalol and cesium chloride) ${ }^{20}$. A recent study by Damiano et al. has revealed that the induction of early afterdepolarization is bradycardia dependent ${ }^{20}$ (figure 4). Sustained rhythms induced by early afterdepolarizations are reset or terminated by premature stimuli, depending upon the maximal diastolic potential.

Longer periods of overdrive stimulation influence the sustained rhythmic activity also in relation to the maximal diastolic potential: the more negative this potential the longer the period overdrive suppression ${ }^{20}$. Triggered rhythms, induced by early afterdepolarizations, behave similarly to abnormal automatic mechanisms in their response to overdrive pacing and extrastimuli ${ }^{20}$.

The clinical relevance of early afterdepolarizations is not well known. Possibly they are involved in the initiation of ventricular tachycardias occurring in the setting of bradycardia and long QT-interval ${ }^{8}$.

\section{Delayed afterdepolarizations}

Delayed afterdepolarizations are oscillations of the diastolic membrane potential occurring after complete repolarization of the preceding action potential. When the amplitude becomes sufficiently high, threshold is attained and a full depolarization will develop. This depolarization is followed by another delayed afterdepolarization (figure 5). In such a way sustained rhythmic activity can occur ${ }^{100}$. Delayed afterdepolarization have been demonstrated in normal cardiac tissues, such as coronary sinus ${ }^{151}$ and atrioventricular valve tissue ${ }^{150}$, and also in atrial tissues from diseased human hearts and in ventricular specialized conducting ${ }^{35,98}$ and myocardial tissues ${ }^{34}$ from normal or 

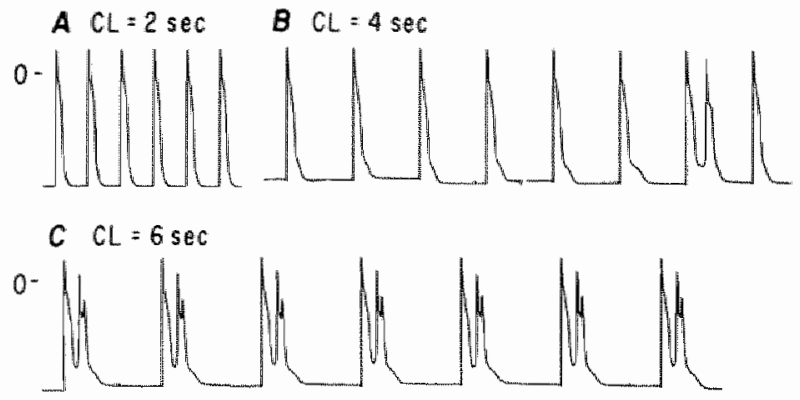

D CL $=10 \mathrm{sec}$

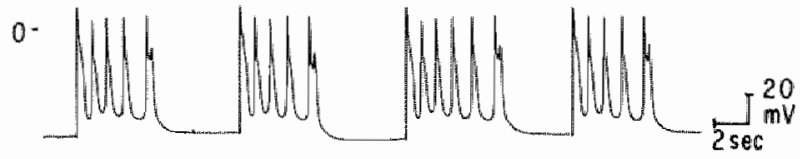

Figure 4

EADs at high membrane potentials and resultant triggered activity. Records were obtained 40 minutes after exposure to cesiun ( $5 \mathrm{mM}$ ) with $2 \mathrm{mM}$ potassium. See text for description. CL $=$ cycle length. (from ref. 20 , printed with permission from the American Heart Association, Inc).

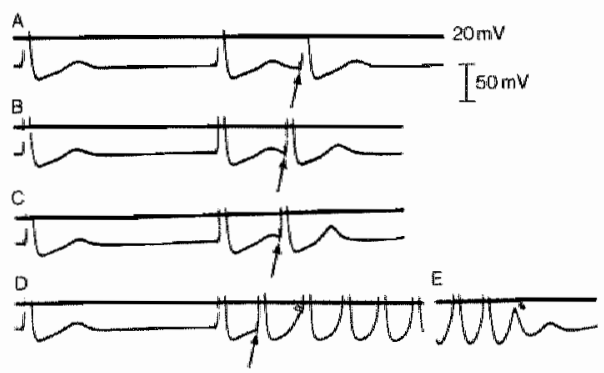

Figure 5

Triggered sustained rhythmic activity in a mitral valve thber induced by a premat ure stimulus. Ontly the lowe: part of the action potentials recorded from the same liber are shown in each panel. The top trace in ench pancel (100 msec time pips) is at $-20 \mathrm{mV}$. The preparation is being perfused with $1.0 \mathrm{mg} / \mathrm{ml}$ of epinephaine and is driven at a basic cycle length of $2700 \mathrm{msec}$. In $A$, a premature impulse (arrow) is induced $1200 \mathrm{msec}$ after the last basic impulse, and well after the peak of irs afterdepolarization. The amplitude of afterdepolarization of the premature impulse is the same as that of the basic impulse. In $B$ and $C$, the premature impulse (arrows) is induced progressively earlier on the preceding afterdepolarization, and the amplitude of the afterdepolarization of the premature impulse is increasing. In D, when the premature impulse (solide arrow) is induced prior to the time at which the afterdepolarization of the preceding basic impulse would have occurred, an non-driven action potential arises from the peak of its afterdepolarization (open arrow) and non-driven, sustained rhy hmic activity continues for 15 minutes.

Only the first four action potentials of the sustained rhytmic activity are shown. (from ref. 152, printed with permission from Martinus Nijhoff Publishers).

diseased hearts exposed to toxic concentrations of digitalis. Other circumstances inducing delayed afterdepolarizations are catecholamines ${ }^{134}$, myocardial infarction ${ }^{22}$ and sodium free ${ }^{17}$, calcium rich ${ }^{131}$ solutions.

Increasing the preceding pacing rate increases the amplitude of delayed 
afterdepolarizations and decreases the coupling interval to the last stimulated action potential $35,101,151$

Aso single extrastimuli increase the amplitude and decrease the coupling interval 35,151 . In this regard delayed afterdepolarizations behave differently from early afterdepolarizations and abnormal automaticity.

\section{The mechanism of delayed afterdepolarizations}

The mechanism of delayed afterdepolarizations is not fully understood. In the setting of digitalis intoxication it is thought that, through inhibition of the sodium potassium ATP-ase, sodium accumulates within the cell. This depresses the sodium-calcium exchange by which also the intracellular calcium concentration increases.

Increased intracellular calcium concentrations can also occur in the presence of catecholamines, following fast drives, and in sodium free and/or calcium rich solutions ${ }^{89}$. When the sarcoplasmic reticulum becomes overloaded it releases calcium in an oscillatory fashion. This increases monovalent cation conductance, inducing an transient inward sodium current, which is thought to be responsible for the delayed afterdepolarization ${ }^{1 / 7}$. The described mechanism allows different possibilities to influence delayed afterdepolarizations ${ }^{99}$. Callum overload may be prevented by calcium antagonists and beta-blockade, whereas drugs like lidocaine, phenytoin and tetrodotoxin inhibit the ransient inward current.

\section{Abnormal automaticity}

Abnormal automaticity is defined as spontaneous impulse generation from a reduced maximal diastolic potential in cardiac fibers, normally showing a high diastolic potential 54. The ionic mechanism for phase 4 depolarization in abnormal automaticity is probably different from the mechanism at high resting potentials, but it has not been precisely defined ${ }^{37}$. It has been suggested that an increase in sodium ${ }^{90}$ and/or a decrease in repolarizing potassium current plays a role ${ }^{61}$. Also an inward calcium current has been held responsible for the diastolic depolarization ${ }^{2 !}$.

Diastolic depolarization leading to abnormal automaticity can be achieved by application of intracellular currents ${ }^{60}$, exposure to barium salts ${ }^{115}$, in 24 hour infarct Purkinje fibers 22 and in digitalized Purkinje fibers ${ }^{98}$.

To some extent the slope of phase 4 depolarization and the rate of impulse generation are a function of the value of the maximal diastollic potential ${ }^{55}$ : a lower maximal diastolic potential ustually is associated with a more rapid rate ${ }^{22,55}$.

Also the effect of overdrive stimulation depends on the diastolic membrane potential and on the duration and rate of overdrive ${ }^{22}$. In a recent study ${ }^{22}$, three types of automaticity were recognized, according to the value of the diastolic membrane potential: high potential automaticity ( $<-70 \mathrm{mv}$ ), intermediate potential automaticity (between -61 and $-70 \mathrm{mv}$ ) and low potential automaticity $(>-60 \mathrm{mv})$. Short periods of overdrive ( 15 seconds or 30 beats) resulted in marked suppression of high potential automaticity, slight suppressing of intermediate potential automaticity and little or no suppression of low potential automaticity. The extent of overdrive suppression of intermediate potential automaticity was related to the rate and duration of the overdrive train and the amount of hyperpolarization that occurred in the pacemaker fiber. Low potential automaticity was not owerdrive suppressible, even after periods of up to 5 minutes. These results are 
presumedly related to the fact that at low membrane potentials the upstroke of the action potential is primarily calcium dependent and as a result less sodium enters the cell to prime the pump ${ }^{22}$.

Premature impulses have been found not to influence the return cycle length during spontaneous activity due to abnormal automaticity ${ }^{55}$.

A bnormal automaticity can be blocked by drugs like acetylcholine, verapamil, nifedipine and ethmozin ${ }^{55}$.

In contrast, lidocaïne in therapeutic concentrations does not exert any significant effect on the slope of phase 4 depolarization or rate of impulse generation 55 .

\section{The behaviour of abnormal cardiac pacemakers following overdrive}

\section{Overdrive excitation}

Whereas normal cardiac pacemakers show overdrive suppression when exposed to a faster rhythm, this relationship is different in abnormal cardiac pacemakers. Frequently pacemaker foci become activated or accelerated by the faster rhythm, a phenomenon, which has been termed overdrive excitation ${ }^{131}$, overdrive enhancement ${ }^{22}$ or overdrive acceleration.

It has been described in isolated Purkinje fibers which were depolarized or exposed to norepinephrine ${ }^{123}$ or digitalis ${ }^{35}$, and also in the intact dog heart, following induction of acule total atrioventricular block ${ }^{59,125,128}$ or during digitalis intoxication ${ }^{42}$.

Overdrive excitation has to be considered as a general descriptive term irrespective of the underlying mechanism (triggered activity, abnormal automaticity, enhanced normal automaticity). An important finding is that overdrive suppression and overdrive excitation can coexist ${ }^{131}$.

Examples are the induction of overdrive excitation following short drivesand suppression following long drives in dogs with acute atrioventricular block ${ }^{131}$ and in slightly depolarized isolated Purkinje fibers ${ }^{22}$.

While intracellular sodium accumulation plays an important role in the occurrence of overdrive suppression, overdrive excitation is more affected by an increased intracellular calcium content ${ }^{13 \|}$. As bas been discussed before, an increased intracellular calcium overload leads to the induction of delayed afterdepolarizations. According to Vassalle ${ }^{131}$ it may also induce a slow afterdepolarization following a fast driven rhythm.

This slow afterdepolarization can, like a delayed afterdepolarization, reach threshold, leading to a full depolarization.

The ionic mechanism for the slow afterdepolarization probably also depends from the intracellular calcium overload ${ }^{131}$. Calcium becomes extruded through an electrogenic sadium - calcium exchange leading to a non-oscillatory depolarizing current (creep current, tail current) ${ }^{131}$.

Whereas the delayed afterdepolarization is induced by an oscillatory release of calcium from the sarcoplasmic reticulum, the tail current is presumably induced by extrusion from calcium from the cytoplasma ${ }^{\| 31}$. 


\section{Cardiac arrhythmias and digitalis}

Rosen and coworkers have studied extensively in vitro digitalis induced impulse formation, especially in relation to possible implications for clinically occurring arrhythmias ${ }^{2}, 98 \%$.

In a therapeutic dose digitalis causes in the intact heart a slowing of the sinus rate, a variable degree of enhancement of intra-atrial conduction, depression of atrioventricular conduction and prolongation of the atrioventricular nodal effective refractory period ${ }^{98}$. These effects are largely the result of the cholinergic and anti-adrenergic action of digitalis ${ }^{98}$. The cholinomimetic effects of digitalis appear to have no significant role with regard to digitalis action on the ventricle ${ }^{98}$.

In contrast toxic amounts of digitalis profoundly influence the action potential of ventricular cells. This influence is seen earlier in the specialized intraventricular conduction tissue than in the myocardium ${ }^{57}$. Both resting membrane potential, action potential amplitude and the upstroke velocity of the depolarization phase decrease. These effects are largely due to the inhibition of the sodium-potassium pump ${ }^{98}$. The decrease in the diastolic potential may lead to enhanced phase 4 depolarization and thus to enhanced impulse formation ${ }^{98}$.

As discussed before, digitalis intoxication also results in delayed afterdepolarizations. Characteristic for digitalis intoxication is that usually more than one delayed afterdepolarization is observed ${ }^{18,35,98}$ (figures 6 and 7 ).

To initiate an action potential by delayed afterdepolarizations during digitalis toxicity the coupling interval and the amplitude of the delayed afterdepolarizations are of importance 35,98. The height of the amplitude determines whether an action potential is induced and the coupling interval when this action potential occurs.

Amplitude and coupling interval have been studied in the isolated Purkinje fiber by way of programmed electrical stimulation $17,32,48,87,97,99,160,101$. Using this method the rate and duration of the rhythm preceding the delayed afterdepolarization can be varied systematically.

Factors known to influence occurrence and amplitude of delayed afterdepolarizations are lisred in table 1.

Table 1

Factors influencing delayed afterdepolarizations.

1. The inverval duration of the preceding fhythm $32,43,57,97$.

2. The number of preceding action potentials ${ }^{32}$.

3. The duration of the last interval following a regular preceding thythm ${ }^{32}$.

4. The sequence number of delayed afterdepolarizations ${ }^{32,004}$.

5. The diastolic membrane potential ${ }^{36}$.

6. The tension on the Purkinje fiber ${ }^{34}$.

7 . The level of digitalis intoxication.

8. The origin of the Purkinje fiber, whether from the right or the left ventricle ${ }^{94}$.

9. The ionic environment ${ }^{3}$.

10. The presence of drugs ${ }^{50,9 \%}$. 


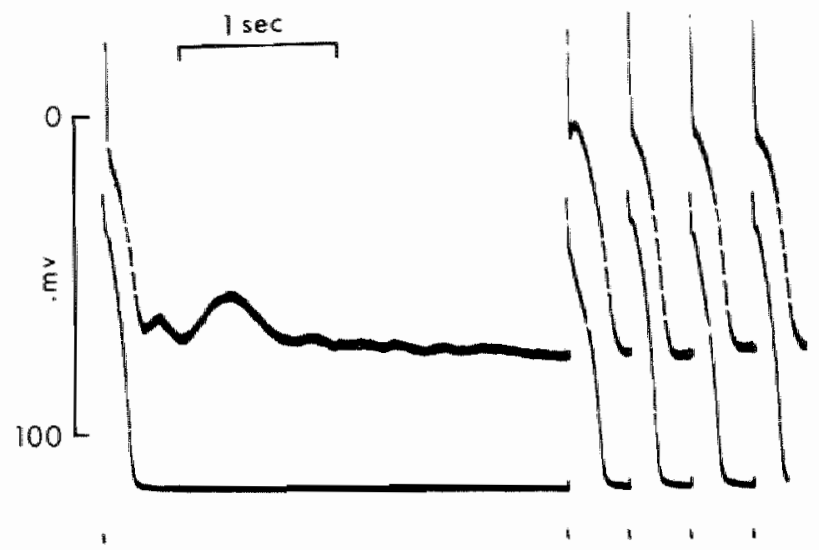

Figure 6

Effect of acetylstrophantidin ( $1 \times 10^{-7} \mathrm{~g} / \mathrm{ml}$ ) on the transmembrane potentals of Purkinge fiburs (nop trace) and muscle (bottom trace) (false tendon-papillary muscle preparation). The first action potential is the last of at tain of ten driven potencials (stimulus artefacts are shown below the bottom traces stimuli delivered to muscle). During the pause in stimulation, two transient depolarizations coupled to the last action potential ocur in the Purkinje fiber but not in the muscle. Spikes were retouched. (from ref. 32, printed with permission from the American Heart Association, [nc).

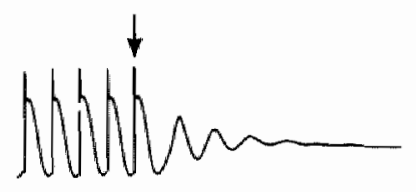

A

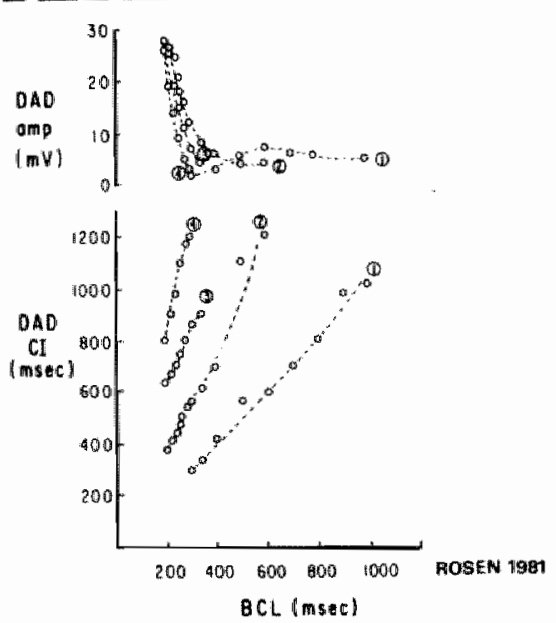

Figure 7

A. Digitalis-induced delayed after-depolarizations (DAD). The Purkinje fiber is simulated for the first five beats. The stimulus is discontinued at the arrow. A series of delayed afterdepolarizanions, gradually decreasing in magnitude, then occurs. Time marks $=1 \mathrm{sec}$. Vertical calibration $=25 \mathrm{mv}$.

B. Relationship of coupling interval (CD) and amplitude (amp) of digitalis-induced DA D to the basic drive cycle length ( $\mathrm{BCL}$ ). The numbers 1 - 4 indicate the four $\mathrm{DAD}$ that follow the last ariver action potential in a train and the curves relating their amp and $\mathrm{CI}$ to $\mathrm{BCL}$ (from ref 100 , printed with permission from $\mathrm{G}, \mathrm{K}$. Hall Medical Publishers). 
The relation of the interval duration, the number of preceding action potentials and the influence of the last interval following a regular rhythm is outlined in detail in chapter 3. Summarizing briefly, it has been found in isolated Purkinje fibers, that when the number of preceding action potentials is increased, the amplitude of the delayed afterdepolarizations shows a marked increase after 1 to 7 preceding action potentials. At higher numbers of stimuli this increase levels off ${ }^{32}$. Shortening of the interval duration of the preceding rhythm results in shortening of the coupling interval of delayed afterdepolarizations. The first delayed afterdepolarization has a coupling interval equal to the preceding interval duration, the second one being twice the preceding interval duration and so on ${ }^{35,100}$. The amplitude of the first delayed afterdepolarization shows an increase on decreasing the preceding interval from 1000 to $500 \mathrm{~ms}$. Following shorter intervals the amplitude rapidly decreases. The second to fourth delayed afterdepolarizations show the opposite behaviour: they increase at shorter preceding intervals reaching a maximum at 200 ms intervals $^{35,100}$ (figure 7).

Changing the last interval only results in a linear relation with the coupling interval ${ }^{35}$ and also the amplitude behaves similarly as described following a regular rhythm.

Delayed afterdepolarizations reaching threshold show a similar behaviour as described above ${ }^{87}$ (figure 8 ).

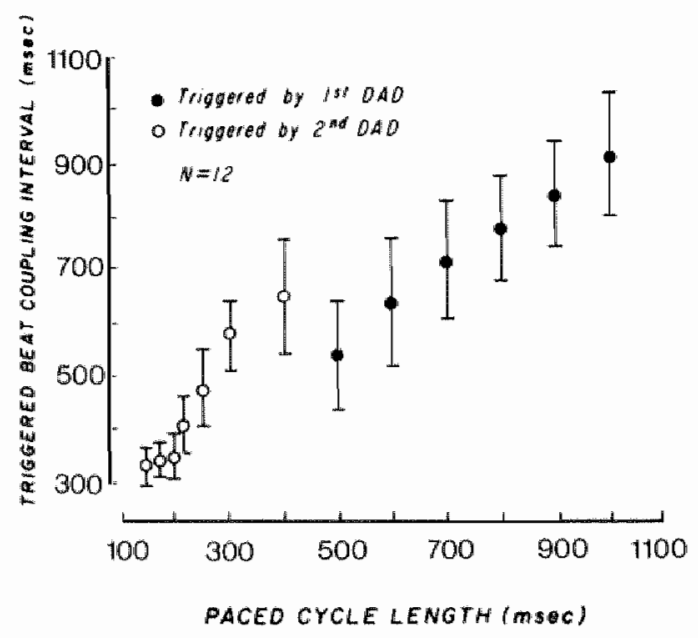

Figure 8

Effect of pacing at different cycle lengths for 15 sec on the coupling interval of the first triggered beat that followed termination of pacing. The filled circles represent those bears that were triggered by the first defayed afterdepolarization (DAD), and the unfilled circles display those beats that were triggered by the second delayed afterdepolarization (from ref. 87, printed with permission from the American Heart Association, Inc). 
These characteristics of delayed afterdepolarizations provide the basis for our study on the role of triggered activity in ventricular arrhythmias in the intact heart (chapters 3-5). The following characteristics of DAD induced ectopic activity were expected ${ }^{100} ; 1$ ) a direct linear relation between the pacing interval and the first postpacing interval; 2$)$ after short pacing intervals $(<500 \mathrm{~ms}$ ) the first postpacing interval should be twice the pacing interval, because the amplitude of the first DAD is low and the amplitude of the second DAD is high. 3) At long pacing intervals (>500 ms) the interval to the induced QRScomplex is equal to the pacing interval, because the amplitude of the first DAD is high in this interval range.

At low levels of digitalis intoxication threshold is expected to be attained following short pacing intervals because the amplitude is highest in this interval range.

Changing the last interval only may induce comparable changes to the first postpacing interval as regular pacing. 



\section{Extrastimulus related shortening of the first postpacing interval in digitalis induced ventricular tachycardia.}

\section{Observations during programmed electrical stimulation in the conscious dog.}

by

Anton PM Gorgels, MD, Henrierte DM Beekman, Pedro Brugada, MD, Willem RM Dassen, PhD, David AB Richards, FRACP*, Hein JJ Wellens, MD, FACC. Department of Cardiology, University of Limbarg, Annadal Hospital, Maastricht, The Netherlands

* Supported by Overseas Research Fellowships of the National Heart Foundation of Australia and Royal Australasian College of Physicians

Published in the Journal of the American College of Cardiology 1983; 1: 840-857.

\section{Abstract}

The effect of different modes of pacing on interval and configuration of the first postpacing QRS-complex was studied during digitalis-induced ventricular tachycardia in the conscious dog. The effect of overdrive pacing was related to pacing rate; the longest pacing intervals resulted in prolongation of the first postpacing interval, while increasing the rate of overdrive pacing led to a progressive shortening of the first postpacing interval. When extrastimuli were introduced during fixed rate pacing, the duration of the first postpacing interval was found to be predominantly affected by the extrastimulus coupling interval. The importance of the last paced interval to the duration of the first postpacing cycle length was also observed when only a single or two extrastimuli were given.

The duration of the first postpacing interval was found to be independent of the site of stimulation. In contrast, the configuration of the first postpacing QRS-complex was found to be related to the site of pacing; the first postpacing QRS-complex originated close to the site of stimulation independent of the configuration of the tachycardia. In conclusion, it was found that during digitalis induced ventricullar tachycardia $I$ ) the first postpacing interval is mainly dependent on the interval of the last paced beat, 2 ) the length of the first postpacing interval is independent of the site of stimulation, but 3 ) the morphology of the first postpacing QRScomplex is related to the site of stimulation.

These findings may facilitate the understanding of complex ventricular arrhyth mias observed during severe digitalis intoxication in human beings.

Over the last two decades many characteristics of digitalis induced arrhythmias have been described. Clinically, digitalis-induced ventricular tachycardias characteristically show a narrow QRS width, a configuration suggesting an origin in the specialized conduction 
system ${ }^{140}$ and a rate varying between $140-180$ beats $/ \mathrm{min}$. That digitalis-induced ventricular arrhythmias originate in the specialized conduction system is supported by studies in the intact dog heart ${ }^{19,71,97}$ and in isolated Purkinje fibers ${ }^{94,97,119}$. The left ventricular Purkinje system has a greater sensitivity for digitalis glycosides than the right-sided Purkinje tissue ${ }^{19,71,94}$, a finding that probably explains the prevalence of right bundle branch block configuration in many clinically occurring digitalis-induced ventricular tachycardias.

Electrical stimulation studies in the intact dog heart have elucidated many important characteristics of digitalis-ind uced arrhythmias. The specialized conduction system in the ventricles has the property of intrinsic automaticity due to phase 4 depolarization. During digitalis administration depression of normal automaticity is the most common finding 120,159 . Abnormal automaticity is defined as impulse generation from phase 4 depolarization arising from a reduced maximum diastolic potential ${ }^{54}$. Digitalis toxicity results in a decrease of the maximum diastolic potential ${ }^{57,86,97,98}$. Overdrive pacing results in suppression of phase 4 depolarization ${ }^{32,99}$. However, an increase in the slope of phase 4 depolarization is sometimes observed ${ }^{\text {g9 }}$.

Triggered activity is defined as the generation of one or more impulses as a consequence of a prior impulse ${ }^{54}$. The occurrence of triggered activity during toxic amounts of digitalis administration has been substantiated by studies in the intact heart. Vassalle et al ${ }^{120}$ showed that sinus beats could trigger ventricular extrasystoles. However, sinus beats were unable to terminate runs of extrasystoles.

Castellanos et al ${ }^{12}$ and Lown et al ${ }^{80,81}$ found that one or more ventricular impulses could be elicited after ventricular stimulation. Increases in atrial ${ }^{47,159}$ or ventricular ${ }^{160,161}$ rate enhanced the ventricular automaticity. This enhancement became apparent as a shortening of the duration of the first postpacing interval, an increase in the number of the ectopic complexes and an increase in duration of time that ectopic impulse generation could be elicited during dissipation of digitalis intoxication.

The finding at the cellular level of delayed afterdepolarizations seems to explain many of these observations $17,18,32,35,48,97,98$. These afterdepolarizations can reach threshold leading to spontaneous arrhythmias following a paced rhythm. Coupling interval and amplitude of afterdepolarizations are dependent on the number of stimulated action potentials, the rate of drive and the degree of digitalis intoxication. An extrastimulus following regular drive causes additional shortening of the coupling intervals of afterdepolarizations. The dependency of the rate of drive and the extrastimulus interval on the first postpacing interval of ventricular ectopic complexes had also been shown in the intact dog heart, suggesting that afterdepolarizations, indeed, play a role in the occurrence of manifest ectopic activity during digitalis intoxication ${ }^{74,161}$

These studies, however, were performed at relatively low levels of digitalis intoxication, at a stage before sustained ventricular tachycardia became manifest spontaneously. Little is known about the effect of electrical stimulation during sustained ventricular tachycardia. Thus, it is not known whether these same phenomena are present during sustained ventricular tachycardia induced by digitalis. We hypothesized that if delayed afterdepolarizations are also the cellular basis for sustained ventricular tachycardia induced by digitalis intoxication, a relation between the rate of overdrive pacing and the postpacing interval is to be expected; that is, the higher the rate of overdrive pacing, the shorter the first postpacing interval. Additional acceleration of the first postpacing 
interval is to be expected, depending on the prematurity of the extrastimulus interval. In the present study we report on the effect of different modes of stimulation on interval and configuration of the first postpacing QRS-complex during digitalis-induced ventricular tachycardia in the conscious dog. Data are presented on the effect of single and paired ventricular stimuli and of overdrive pacing at different rates with and without an extrastimulus.

\section{Methods}

\section{Preparation of Study Dogs}

Experiments were performed in six dogs especially prepared for electrophysiologic study in the conscious nonsedated state. After a right thoracotomy, bipolar electrodes were screwed intramurally into the left ventricular apex in three dogs. In three other dogs unipolar electrodes were sewn onto the left ventricular apex. The last three dogs also had bipolar epicardial electrodes sewn on to the right ventricular base. In one dog complete AV block was produced by formalin injection into the AV node ${ }^{114}$.

The electrodes were exteriorized through the neck, aliowing recording and stimulation from different sites. The dogs were studied in a conscious state without premedication 1 to 3 months after recovery from the thoracotomy. Digitalis intoxication was achieved by infusing digoxin $0.1 \mathrm{mg} / \mathrm{kg} / 1-1 / 2 \mathrm{hr}$. Sustained ventricular tachycardia developed about 12 hours after the infusion. The tachycardias lasted 24 to 48 hours, enabling us to perform the stimulation studies under stable circumstances and to repeat the stimulation protocol several times during one experiment. On the day of digitalization, all dogs were clinically unwell with nausea, vomiting and tachypnea. Urine output was normal. On subsequent days (when electrophysiological studies were performed), all dogs were clinically well, without tachypnea and still with normal urinary output.

Before the administration of digoxin the pacing protocol was performed to establish the possibility of initiating tachycardia in the basal state.

Five or six surface electrocardiographic leads (I, II, III, AVR, and AVF or $V_{1}$ and $V_{6}$, , were simultaneously monitored and recorded via a Siemens-Elena ink jet recorder onto paper at 100 or $25 \mathrm{~mm} / \mathrm{sec}$. Stimulation was performed with a Medtronic 5325 stimulator. Bipolar pacing stimuli of 2 ms duration were given at twice diastolic current threshold. In between each episode of stimulation, the dominant tachycardia was given time to recover completely.

\section{Pacing protocol}

\section{Overdrive pacing}

The effect of overdrive pacing on ventricular tachycardia was observed after fixed rate stimulation with nine beats. The rate of overdrive pacing was increased by decreasing the pacing cycle length in steps of 20 or $25 \mathrm{~ms}$. The longest overdrive interval used was only slightly shorter than the mean tachycardia interval.

\section{The overdrive-extrastimulus study}

During these studies, ventricular tachycardia was overpaced by eight stimuli, starting with intervals slightly shorter than the mean tachycardia interval. Overdrive stimulation was followed by one extrastimulus given with increasing prematurity in 
steps of $10 \mathrm{~ms}$ to ventricular refractoriness. A full scan of extrastimuli was given at a constant overdrive cycle length. The protocol was thereafter repeated using increasingly shorter overdrive cycle lengths.

\section{The double extrastimulus study}

During the double extrastimulus study, the first extrastimulus interval was kept constant starting with an interval only slightly shorter than the mean tachycardia interval; the interval of the second extrastimulus interval was shortened in steps of 10 ms to ventricular refractoriness.

A full scan of second stimuli was given with a constant first extrastimulus interval. Thereafter, the scans were repeated by increasing the prematurity of the first test stimulus in steps of 20 or $40 \mathrm{~ms}$.

\section{The single extrastimulus study}

In the single extrastimulus study, a test stimulus was given during ventricular tachycardia with increasing prematurity (in steps of $10 \mathrm{~ms}$ ) to ventricular refractoriness.

The following abbreviations are used:

$\mathrm{V}_{\mathrm{a}} \quad$ : The spontaneously occurring ventricular tachycardia complex.

$V_{1} \quad$ : The first extrastimulus.

$V_{2} \quad$ : The second extrastimulus.

$\mathrm{V}_{\mathrm{s}} \quad$ : The ventricular complexes induced during overdrive pacing.

$\mathrm{V}$ : The first postpacing complex.

S : The stimulus artefact.

The following intervals were measured:

1. $\mathrm{V}_{\mathrm{z}}-\mathrm{V}_{\mathrm{a}}$ : The last ventricular tachycardia cycle before pacing. From these intervals the mean tachycardia interval was estimated.

2. $V_{s}-V_{s}$ : The interstimulus interval during overdrive pacing.

3. $\mathrm{V}_{\mathrm{s}}-\mathrm{V}$ : The interval between the last ventricular complex during overdrive pacing and the first postpacing QRS-complex.

4. $V_{\mathrm{s}}-\mathrm{V}_{\mathrm{l}}$ : The interval between the last ventricular complex during overdrive pacing and the extrastimulus.

5. $V_{1}-V$ : The interval between the first extrastimulus to the first postpacing QRS-complex.

6. $V_{\mathrm{a}}-\mathrm{V}_{1}$ : The interval between the last tachycardia complex and the first extrastimulus.

7. $V_{1}-V_{2}$ : The interval between the first and the second extrastimulus.

8. $V_{2}-\mathrm{V}$ : The interval between the second extrastimulus and the first postpacing complex. 


\section{Specific studies}

We specifically studied the following features:

During overdrive stimulation: The influence of rate and site of overdrive stimulation on the first postpacing tachycardia interval and QRS-configuration.

During the overdrive extrastimulus study: 1) The influence of an increase in premaru rity of the extrastimulus $\left(V_{s}-V_{1}\right)$ on the first postpacing interval $\left.\left(V_{1}-V\right) .2\right)$ The influence of overdrive stimulation at decreasing intervals $\left(V_{s}-V_{s}\right)$ on the first postpacing interwal $\left(V_{1}\right.$ - V). 3) The influence of stimulation at different sites of the ventricles (basal right ventricle versus left ventricular apex) on the first postpacing QRS-configuration and time intervals.

During the double extrastimulus study: 1) The influence of an increase in the prematurity of the second extrastimulus $\left(V_{1}-V_{2}\right)$ on the first postpacing interval $\left(V_{2}-V\right)$.

2) The influence of an increase in prematurity of the first extrastimulus $\left(V_{a}-V_{1}\right)$ on the first postpacing interval $V_{2}-V$ ). 3) The influence of stimulation on the first postpacing QRS-configuration.

During the single extrastimulus study: 1) The influence of an increase in prematurity of the test stimulus $\left(V_{a}-V_{1}\right)$ on the first postpacing interval $\left(V_{1}-V\right)$.2) The influence of stimulation on the first postpacing QRS-configuration.

\section{Results}

Prior to the administration of digoxin, no ectopic ventricular activity could be induced following any of the stimulation modes. Ventricular tachycardia was induced by digitalis intoxication in all dogs. The cycle length of the tachycardias varied between 260 and 330 $\mathrm{ms}$ per minute. The majority of ventricular tachycardias had a right bundle branch block configuration. A minority of tachycardias had a left bundle branch block configuration. For the purpose of this study, no attempt was made to further characterize the morphologic features of tachycardia. 

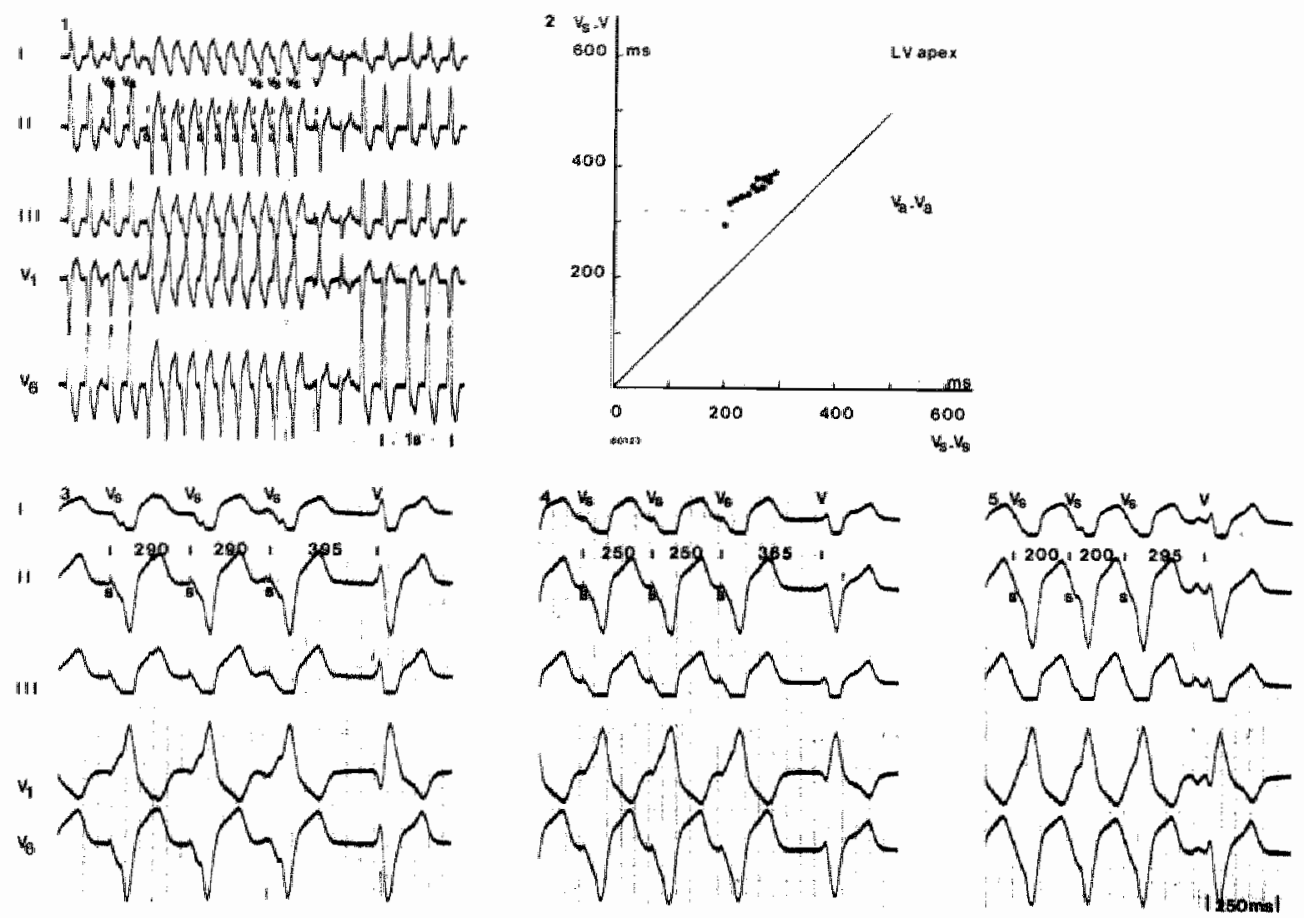

Figume 1.

The overdrive experiment

See text

\section{Overdrive experiments}

Representative overdrive experiment (Fig. I).

Panel 1 of this figure shows the procedure at a paper speed of $25 \mathrm{~mm} / \mathrm{s}$. The last four complexes of the ventricular tachycardia before pacing have a left bundle branch block configuration, with a mean $V_{a}-V_{a}$ interval of $320 \mathrm{~ms}$. After these four complexes, the tachycardin is overpaced with nine stimuli at the left ventricular apex. The first two complexes following cessation of pacing have a similar configuration similar to that of the paced complexes, that is, a pattern of right bundle branch block with left axis deviation. Thereafter the original tachycardia recurs.

Panel 2 is a diagram showing the behaviour of the first postpacing interval $\left(V_{s}-V\right)$ on increasing the rate of overdrive pacing (shortening of the $V_{s}-V_{s}$ interval). The broken horizontal line represents the mean tachycardia interval $\left(V_{a}-V_{a}=320 \mathrm{~ms}\right)$. The diagram shows that at the slowest overdrive rate, the first postpacing interval is longer than the mean tachycardia interval. By increasing the rate of overdrive pacing (reducing the $V_{s}-$ $V_{s}$ intervals) a decrease of this prolongation results. The first postpacing interval becomes shorter than the mean ventricular tachycardia interval at a very high overdrive pacing rate (a $V_{s}-V_{s}$ interval of $200 \mathrm{~ms}$ ).

Panels 3,4 and 5 show three selected portions of this experiment. In all three panels, the last three paced complexes and the first postpacing complex are shown. Panel 3 shows 
$\mathrm{V}_{\mathrm{s}}-\mathrm{V}_{\mathrm{s}}$ intervals of $290 \mathrm{~ms}$ that are followed by a postpacing interval of $395 \mathrm{~ms}$. The postpacing QRS-complex has a configuration that has some similarity to the paced complexes although the following differences should be noted: the paced beats show a slow initial upstroke (the delta-like beginning of QRS-complex) followed by more rapid spread of ventricular activation, whereas the postpacing QRS-complex has a sharp initial deflection.

Panel 4 shows $V_{s}-V_{s}$ intervals of $250 \mathrm{~ms}$ with a first postpacing interval of $365 \mathrm{msec}$. In panel $5, V_{s}-V_{s}$ intervals of $200 \mathrm{~ms}$ were used and a first postpacing interval of $295 \mathrm{~ms}$ was measured. The configuration of the first spontaneous postpacing QRS-complexes is identical to that of panel 3 .

Similar abservations on the behaviour of the first postpacing interval on decreasing the $V_{s}$ $-V_{\mathrm{s}}$ interval were made in all experiments. In one experiment, overdrive pacing was performed with $V_{s}-V_{s}$ intervals below $200 \mathrm{~ms}$. This resulted in first postpacing intervals that were less than the mean $\mathrm{V}_{\mathrm{a}}-\mathrm{V}_{\mathrm{a}}$ interval during ventricular tachycardia.

\section{Overdrive Extrastimulus Experiment}

\section{Effect of extrastimulus (Fig. 2).}

Panel $l$ of this figure shows the last seven complexes of a ventricular tachycardia before pacing. The ventricular tachycardia has a left bundle branch block configuration and a vertical axis. The mean ventricular tachycardia interval is $260 \mathrm{~ms}$. Pacing at the right ventricular base comprised 8 stimuli with equal cycle lengths $\left(V_{s}-V_{s}=230 \mathrm{~ms}\right)$ followed by a single extrastimulus $\left(V_{\mathrm{s}}-\mathrm{V}_{1}\right)$.

The paced complexes have a $Q R S$ configuration almost identical to that of the tachycardia. After pacing, the original tachycardia recurs, the first spontaneous tachycardia complex (V) also shows the same QRS-configuration. All first postpacing complexes during the experiment showed the same QRS-configuration.

Panel 2 is a diagram illustrating the relation between the prematurity of the extrastimulus $\left(V_{s}-V_{1}\right)$ and the first postpacing interval $\left(V_{1}-V\right)$. The broken horizontal line represents the mean intrinsic tachycardia interval $\left(\mathrm{V}_{\mathrm{a}}-\mathrm{V}_{\mathrm{a}}=260 \mathrm{msec}\right)$. A similar relationship to that observed in Figure 1 exists between the prematurity of the extrastimulus and the first postpacing interval. At the longest extrastimulus intervals there is prolongation of the first postpacing interval compared to the mean tachycardia interval. On increasing the prematurity of the extrastimulus, shortening of the first postpacing interval occurs, reaching values close to the mean spontaneous tachycardia interval.

Thus, shortening of the first postpacing interval occurs not only after fixed rate pacing, but also following a single extrastimulus. Similar observations were made in all experiments. At very short extrastimulus intervals, the first postpacing interval became less than the mean tachycardia interval.

Panel 3 shows the same procedure in this experiment, but now during pacing of the left ventricular apex. The paced complexes have a right bundle branch block configuration with left axis deviation. After pacing, the configuration of the first three complexes is similar to that of the paced complexes. Thereafter postpacing complexes 4,6 and 7 suggest fusion between the early postpacing QRS-complexes and the original tachycardia complexes. After these complexes, the original tachycardia recurs. 

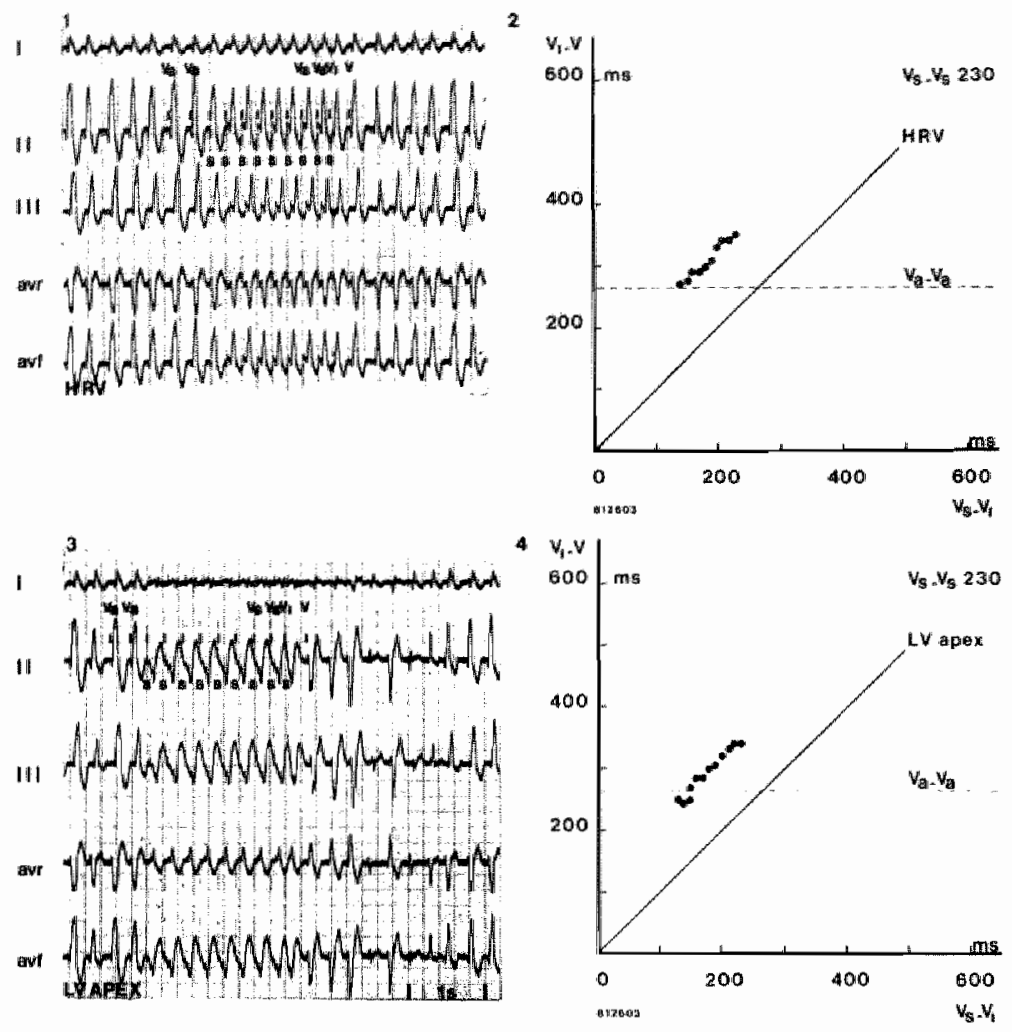

Figure 2

The overdrive extrastimulus experiment. Role of the extrastimulus interval and site of stimulation See text.

All first postpacing complexes had the same configuration after pacing at the left ventricular apex. "These observations on the relation of the site of stimulation and the configuration of the first postpaced QRS-complex suggest that stimulation resulted in initiation of ectopic activity close to the site of stimulation, followed by recurrence of the spontaneous tachycardia.

Panel 4 shows the relation between the prematurity of the extrastimulus interval $\left(V_{s}-V_{1}\right)$ and the first postpacing interval $\left(V_{1}-V\right)$. The arrangement is similar to that in panel 2. An almost identical relation to that in panel 2 appears to be present between the extrastimulus interval and the first postpacing interval. Thus, shortening of the first postpacing interval can be induced by a single extrastimulus during fixed rate ventricullar drive and is independent of the site of stimulation. 


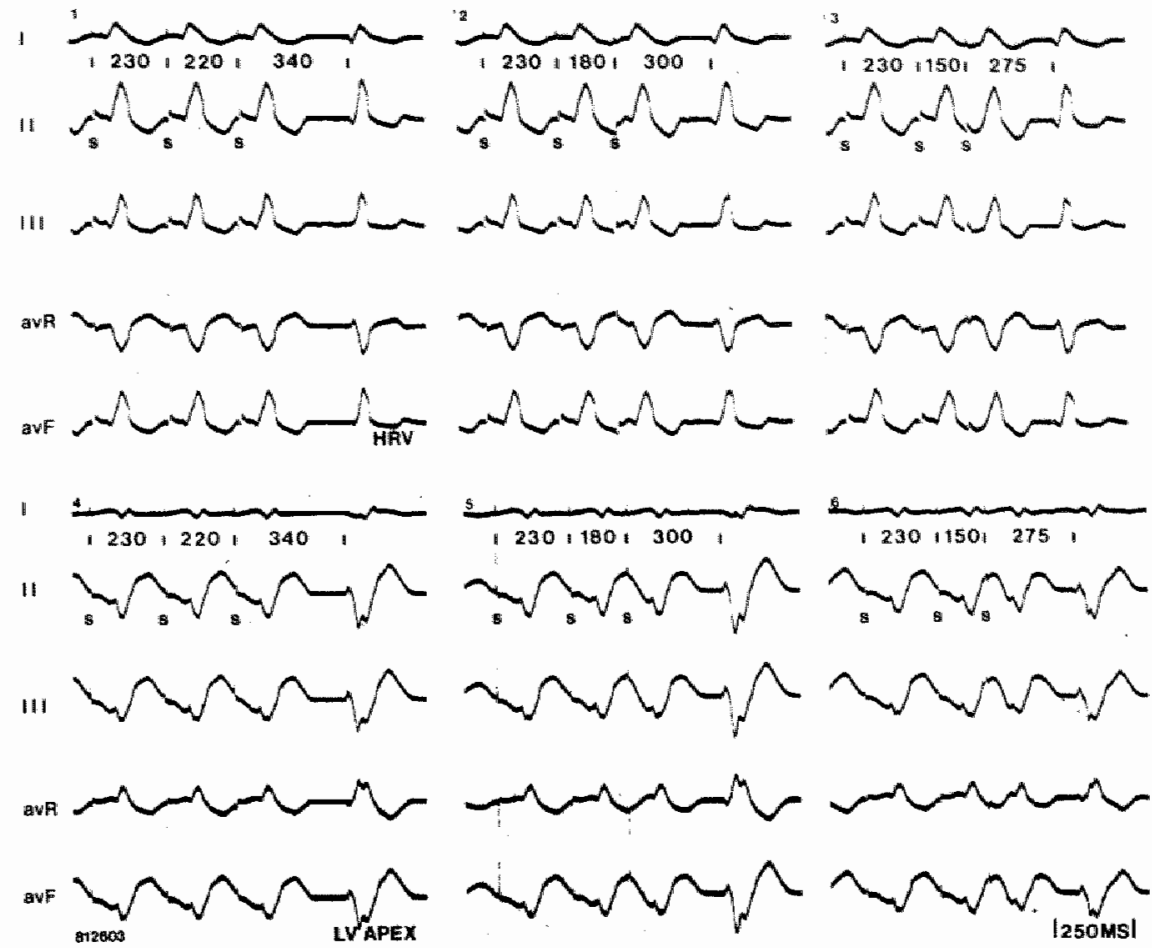

Figure 3

The overdrive-extrastimulus experiment. Role of extrastimulus interval and site of stimulation see text.

Stimulation at different sites of ventricles (Fig. 3).

Figure 3 shows these observations from selected portions of the overdrive extrastimulus experiment in the same dog (as that depicted in Figure 2) at a paper speed of $100 \mathrm{~mm} / \mathrm{sec}$. The top three panels were recorded during pacing of the right ventricular base and the bottom three panels during pacing of the left ventricular apex. The last two overdrive pacing stimuli, the extrastimulus and the first postpacing complex are shown.

Panel 1 shows a first postpacing interval of $340 \mathrm{~ms}$ following an extrastimulus interval of $220 \mathrm{~ms}$.

In panel 2, the first postpacing interval is $300 \mathrm{~ms}$ after an extrastimulus interval of $180 \mathrm{~ms}$. In panel 3 , the first postpacing interval is $275 \mathrm{~ms}$ at an extrastimulus interval of $150 \mathrm{~ms}$.

Panels 4,5 and 6 show exactly the same time relations during pacing of the left ventricular apex. However, the configuration of the QRS-complexes is changed, suggesting postpacing impulse formation close to the site of pacing. All panels, as in Figure 1, show a slow initial deflection followed by more rapid activation of the paced QRS-complexes and sharp initial deflection of the first postpacing QRS-complex. 


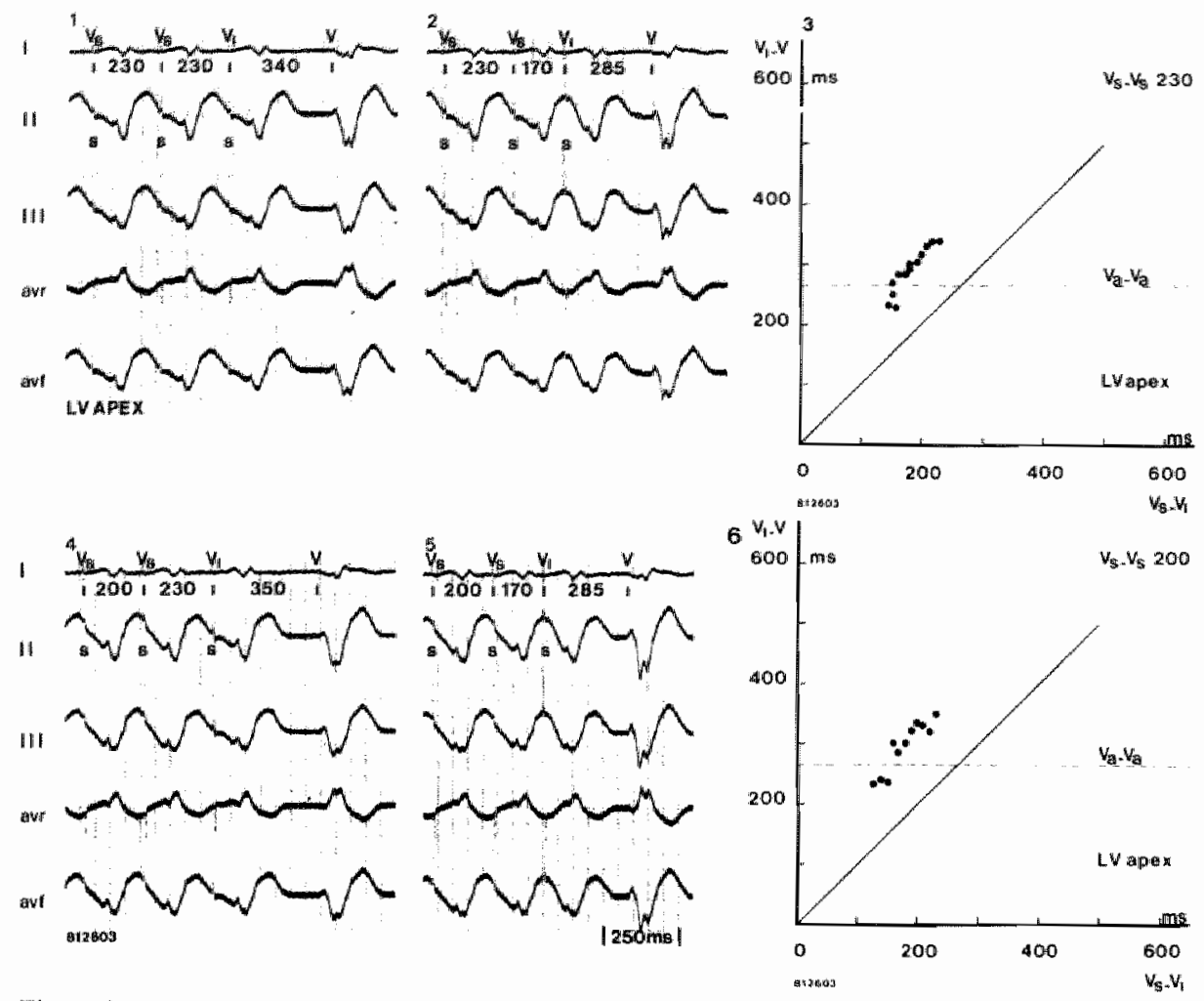

Figure 4

The overdrive-extrastimulus experiment. Role of overdrive pacing See text.

\section{Effect of rate of overdrive pacing during overdrive extrastimulus experiments}

Overdrive extrastimulus studies were performed at different overdrive pacing $\left(V_{s}-V_{s}\right)$ interwals to study the effect of the rate of overdrive pacing.

Figure 4. This figure shows selected recordings from the same experiment in Figures 2 and 3 during pacing of the left ventricular apex. In the top three panels, the overdrive cycle length is $230 \mathrm{~ms}$; in the bottom three panels, it is $200 \mathrm{~ms}$. Panels 1 and 2 and 4 and 5 depict the last two of a train of eight paced complexes, the extrastimulus and the first postpacing complex.

In panels 1 and 4 the extrastimulus interval is $230 \mathrm{~ms}$; in panels 2 and 5 , the extrastimulus interval is $170 \mathrm{~ms}$. Panel 1 shows a first postpacing interval of $340 \mathrm{~ms}$ after an extrastimulus interval of $230 \mathrm{~ms}$. Panel 2 shows a first postpacing interval of $285 \mathrm{~ms}$ after an extrastimulus interval of $170 \mathrm{~ms}$. Therefore, as in figures 2 and 3 , shortening of the first postpacing interval occurs after shortening of the extrastimulus interval. Panel 3 diagrammatically depicts the results for all extrastimuli. An almost linear relation is present between the extrastimulus interval and the first postpacing interval, at very short extrastimulus intervals when the first postpacing interval became shorter than the mean 
tachycardia interval. Panel 4 shows a first postpacing interval of $350 \mathrm{~ms}$ after an extrastimulus interval of $230 \mathrm{~ms}$, which is similar to the first postpacing interval in panel 1. Panel 5 show a first postpacing interval of $285 \mathrm{~ms}$ after an extrastimulus interval of 170 $\mathrm{ms}$, which is similar to the first postpacing interval in panel 2 . In panel 6 , the results for all extrastimuli are presented, showing a similar relation between the extrastimulus interval and the first postpacing interval to that in panel 3 .

These findings indicate that the relation of the first postpacing interval and pacing depends mainly upon the last test stimulus interval. The morphologic features of all first postpacing complexes were similar during this experiment.

QRS-complexes during pacing showed slow initial ventricular excitation followed by a more rapid activation, whereas the first postpacing complexes showed a sharp initial deflection.

The resemblance between the first postpacing complex and the subsequent paced complexes suggests an origin of the postpaced complexes to the site of stimulation.

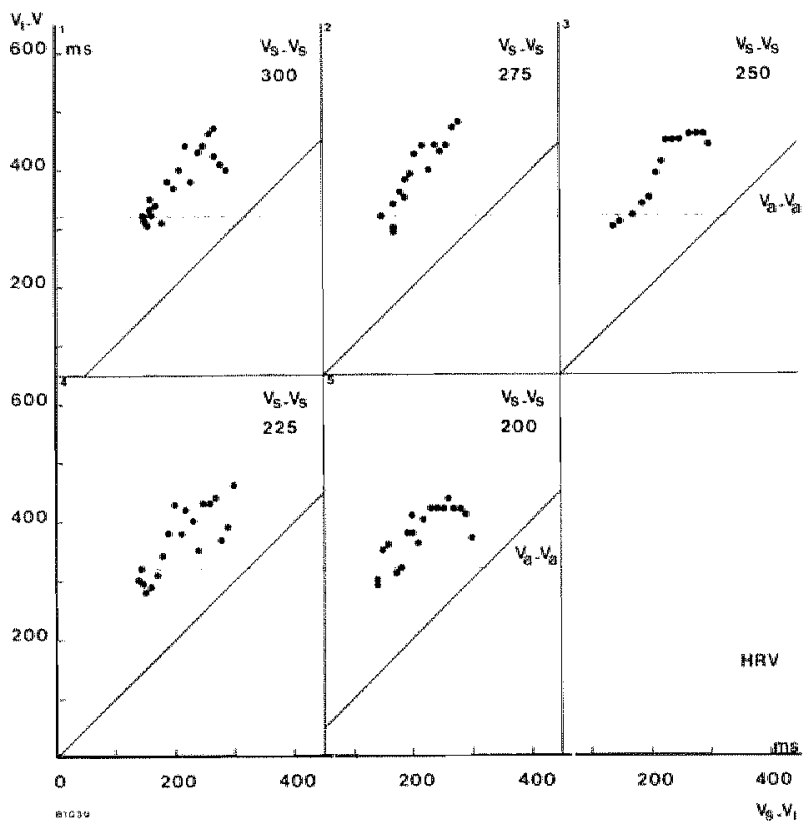

Figure 5

Overdrive extrastimulus experiment See text.

Figure 5. This figure depicts the results of overdrive-extrastimulus experiments during another tachycardia $\left(V_{a}-V_{a}=320 \mathrm{~ms}\right)$. The abscissae depict the extrastimulus intervals $\left(V_{s}-V_{1}\right)$ and ordinates the first postpacing intervals $\left(V_{1}-V\right)$. The mean tachycardia interval is represented as a broken horizontal line. The lines are drawn to facilitate comparison between the curves. The overdrive cycle length is shortened from 300 to 200 ms (panels 1 to 5). All curves show prolongation of the first postpacing interval compared with the mean tachycardia interval, as well as shortening of the first postpacing interval on decreasing the extrastimulus interval. This figure, like Figure 4, suggests that the duration of the first postpacing interval is mainly dependent on the extrastimulus interval. 

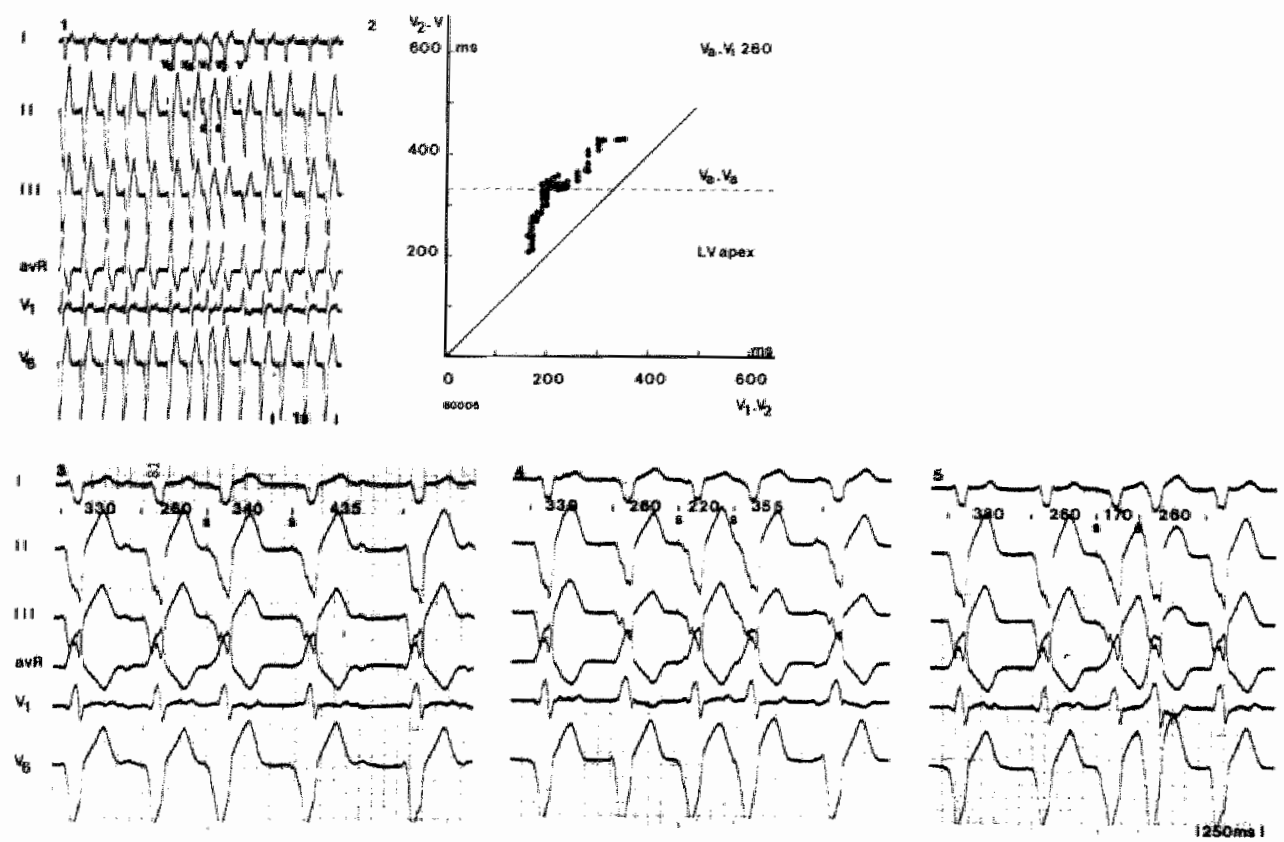

Figure 6

The double extrastimulus experiment. Effect of the second extrastimulus interval during pacing of the lef ventricular apex

See text.

\section{Double Extrastimulus Experiment}

\section{Effect of the second extrastimulus interval (Fig. 6)}

To study the influence of the second extrastimulus on the first postpacing interval, the second extrastimulus interval was progressively shortened in steps of $10 \mathrm{~ms}$ to ventricular refractoriness, and the first extrastimulus interval was kept constant.

Panel 1 of Figure 6 shows the pacing procedure at a paper speed of $25 \mathrm{~mm} / \mathrm{s}$. The tachycardia has a right bundle branch block configuration with marked right axis deviation. The mean ventricular tachycardia interval is $330 \mathrm{~ms}$. Two extrastimuli were given at the left ventricular apex.

Panel 2 shows the influence of the second extrastimulus $\left(V_{1}-V_{2}\right)$ interval on the first postpacing $\left(V_{2}-V\right)$ interval as the first extrastimulus $\left(V_{3}-V_{1}\right)$ interval was kept constant at $260 \mathrm{msec}$. The broken horizontal line represents the mean tachycardia interval $\left(\mathrm{V}_{\mathrm{a}}-\mathrm{V}_{\mathrm{a}}\right.$ $=330 \mathrm{~ms}$.) This panel shows that at long second extrastimulus intervals $\left(V_{1}-V_{2}\right)$ prolongation of the first tachycardia intervals occurs, compared with the mean tachycardia interval. On shortening the second extrastimulus interval, a decrease of the first postpacing interval occurs. When the second stimulus is given very early $\left(V_{1}-V_{2}<200\right.$ ms.), the first postpacing tachycardia interval becomes less than the tachycardia interval. Panels 3,4 and 5 depict three selected portions of the stimulation study at a paperspeed of $100 \mathrm{~ms}$. In all three panels, the last two ventricular tachycardia complexes before pacing, the two extrastimuli and the first postpacing complex are shown. 
In all panels, the tachycardia interval is $330 \mathrm{~ms}$ and the first extrastimulus interval is 260 $\mathrm{ms}$. Panel 3 shows a second extrastimulus interval of $340 \mathrm{~ms}$ followed by a first postpacing interval of $435 \mathrm{~ms}$. Panel 4 shows a second extrastimulus interval of $220 \mathrm{~ms}$ followed by a first postpacing complex at $355 \mathrm{~ms}$. Panel 5 shows a second extrastimulus interval of 170 $\mathrm{ms}$ and a first postpacing complex at $260 \mathrm{~ms}$.

These findings suggest that when two premature stimuli are given during digitalisinduced ventricular tachycardia, shortening of the second extrastimulus coupling interval results in shortening of the first postpacing interval. A comparison between Figure 6 and previous figures shows that a greater amount of shortening of the first postpacing interval could be induced by paired extrastimuli than by single extrastimuli.
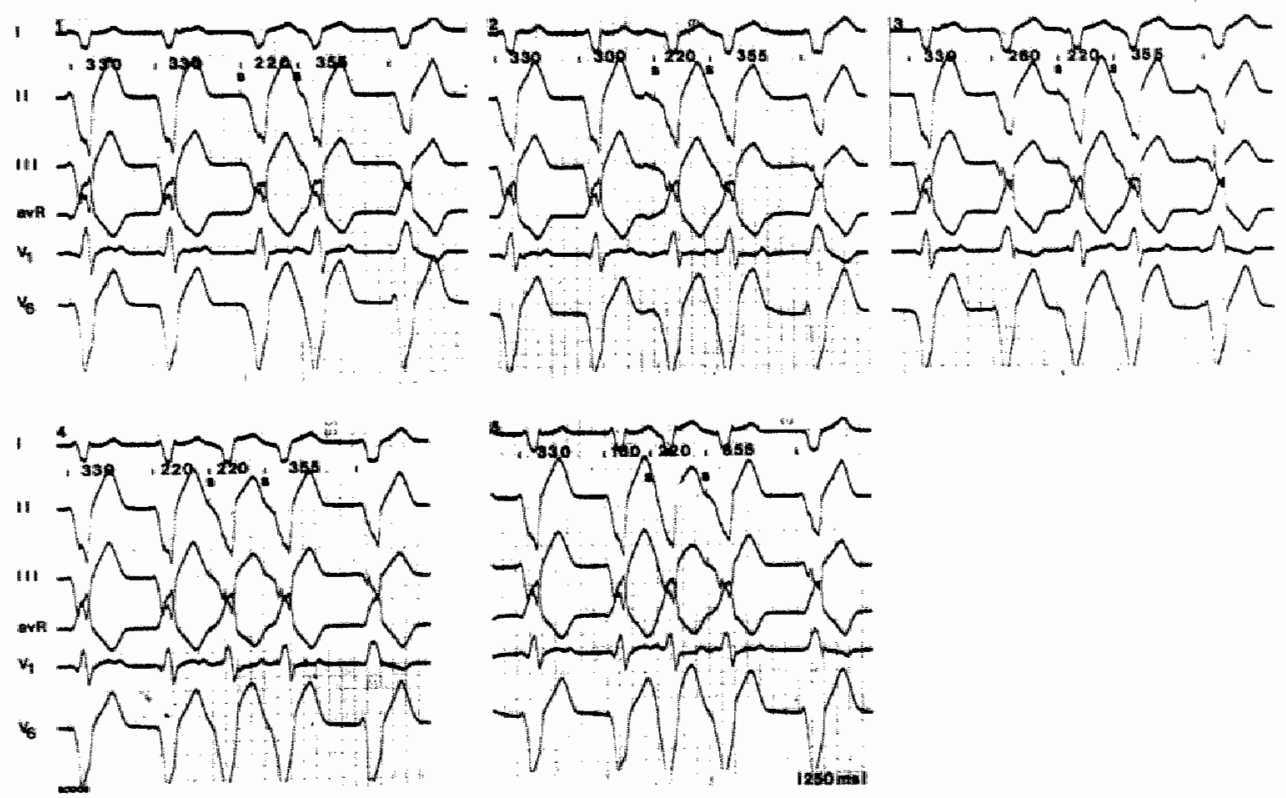

Figure 7

The double extrastimulus experiment. Role of tha first extrastimulus interval See text.

Effect of the First Extrastimulus Interval during the double Extrastimulus Experiment

Stimulation studies using different first extrastimulus intervals $\left(\mathbf{V}_{\mathrm{a}}-\mathbf{V}_{\mathrm{I}}\right)(\mathbf{F i g .} 7$ and 8$)$. In panels $\mathbb{1}$ to 5 of Figure 7 , the first extrastimulus interval is shortened from 330 to 180 ms. The second extrastimulus interval is kept constant at $220 \mathrm{~ms}$. The first postpacing interval remains constant in all panels ( $355 \mathrm{~ms}$ ), suggesting that the first extrastimulus interval does not influence the first postpacing interval. 


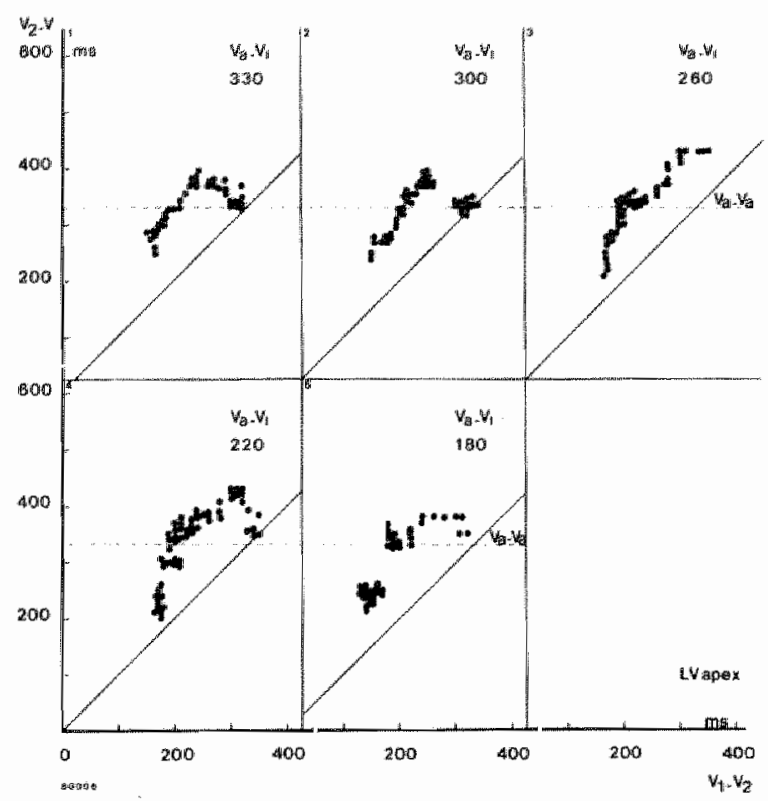

Figure 8

The double extrastimulus experiment

See text.

Figure 8 shows diagrammatically the effect of the duration of the first extrastimulus interval. The abscissae depict the second extrastimulus intervals $\left(V_{1}-V_{2}\right)$ and ordinates the first postpacing intervals $\left(V_{2}-V\right)$. In panels 1 to 5 the first extrastimulus interval is shortened from 330 to $180 \mathrm{~ms}$. The broken horizontal line represents the mean tachycardia interval $\left(V_{a}-V_{a}=330 \mathrm{~ms}\right.$.) These curves essentially show the same behaviour of the first postpacing interval: shortening of the first postpacing interval on increasing the prematurity of the second extrastimulus interval. The first postpacing interval falls below the mean tachycardia interval when the second extrastimulus interval is less than $200 \mathrm{~ms}$. This occurred despite individual differences in response of the first postpacing interval to the second extrastimulus interval.

These observations illustrate that the influence of the first extrastimulus on the first postpacing interval is very small, and confirm that the last paced beat is the main determinant of the first postpacing interval. Most paced complexes had a similar configuration to the complexes of the ventricular tachycardia, as did the first postpacing complexes. In other experiments the paced complexes were different from the ventricular tachycardia morphology, and as in the overdrive and overdrive extrastimulus study, the first postpacing complexes were similar in configuration to the paced complexes. 


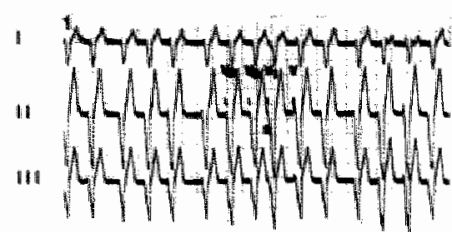

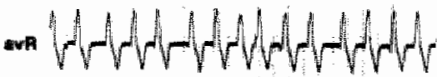

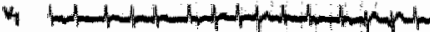

w
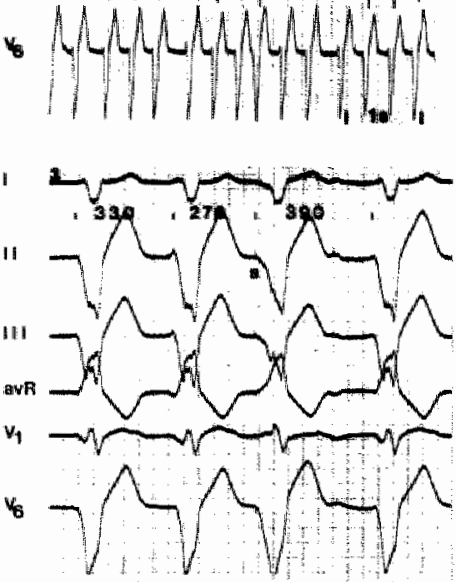

Figure 9

The single extrastimulus experiment

Ser text.
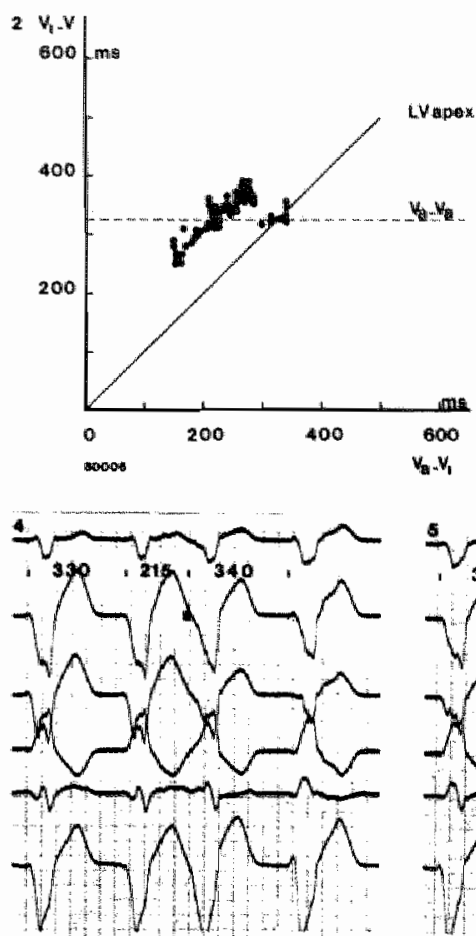

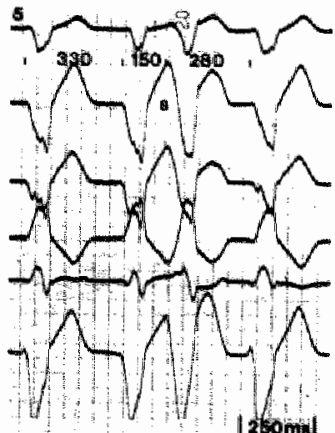

\section{Single Extrastimulus Experiment}

Single extrastimulus given at the left ventricular apex with increasing prematurity to ventricular refractoriness. (Fig. 9).

Panel 1 shows the procedure at a paper speed of $25 \mathrm{~mm} / \mathrm{s}$. Note the irregular rate of the ventricular tachycardia. For analysis $V_{a}-V_{a}$ intervals within $10 \mathrm{~ms}$ of the mean were selected.

Panell 2 illustrates the influence of the extrastimulus $\left(V_{a}-V_{1}\right)$ interval on the first postpacing $\left(\mathrm{V}_{1}-\mathrm{V}\right)$ interval. The broken horizontal line represents the mean tachycardia interval $\left(V_{a}-V_{a}=330 \mathrm{~ms}\right.$.) At very long extrastimulus intervals $\left(V_{a}-V_{1} \geqslant 300 \mathrm{~ms}\right)$ the first postpacing interval was not changed, presumably because impulses from the "dominant tachycardia" occurred before extrastimulus-induced-ectopic activity could become manifest. As the prematurity of the extrastimulus increased, the postpacing interval decreased, ultimately to intervals less than the mean tachycardia cycle length. Panels 3,4 and 5 represent three selected portions of this stimulation study at paper speed of $100 \mathrm{~mm} / \mathrm{s}$. Panel 3 shows that after an extrastimulus at $270 \mathrm{~ms}$ the first postpacing interval $\left(V_{1}-V=390 \mathrm{~ms}\right)$ is longer than the preceding tachycardia interval.

Panel 4 shows the first postpacing interval to be equal to the preceding tachycardia interval $\left(V_{a}-V_{1}=340 \mathrm{~ms}\right)$ after an extrastimulus at $215 \mathrm{~ms}$. Panel 5 shows shortening of the first postpacing interval compared with the preceding tachycardia interval $\left(V_{\|}-V=\right.$ 
$280 \mathrm{~ms}$ ) after an extrastimulus at $150 \mathrm{~ms}$. These observations indicate that an accelerative effect on the first postpaced QRS-complex can already be obtained by delivering a single premature beat during tachycardia.

The paced complexes have a similar configuration to that of the complexes of the ventricular tachycardia as did the first postpacing complexes. In other experiments, the paced complexes were different from the ventricular tachycardia configuration, and as in the overdrive and overdrive-extrastimulus studies, the first postpacing complexes were similar in configuration to the paced complexes.

\section{Discussion}

\section{Configuration of the ventricular tachycardias.}

The majority of the observed ventricular tachycardias had a right bundle branch block configuration, suggesting an origin within the left ventricle. In Figures 6,7 and 9 , examples are given of a tachycardia originating near the left ventricular apex, which has been described as a preferred location for the occurrence of digitalis-induced ventricular tachycardia in the intact dog heart ${ }^{71}$. The higher sensitivity of the left ventricular Purkinje system for digitalis glycosides has been observed by several authors ${ }^{19,71,94}$ and has recently been studied in detail ${ }^{94}$.

Right bundle branch block configuration with right or left axis deviation is seen most commonly in ventricular arrhythmias induced by digitalis in human beings (suggesting an origin within the anterior or posterior fascicle of the left bundle branch ${ }^{140}$ ). In a minority of our experiments, however, a left bundle branch block configuration was also present (Fig. 1 and 2).

\section{Effect of pacing during ventricular tachycardia}

Electrical stimulation of the ventricle results in a characteristic response during digitalisinduced tachycardia.

When pacing is performed close to the origin of the ventricular tachycardia, the QRSconfiguration remains unchanged (Fig. 2, panel 1; Fig. 6,7 and 9). However, when pacing is performed at a different site, the "dominant ventricular tachycardia" is temporarily interrupted by a tachycardia with a configuration dependent on the site of stimulation (Fig. 1, panel 1,; Fig. 2, panel 3). This different tachycardia could be induced by a single extrastimulus and persisted for a few beats after which the original tachycardia resumed.

Complexes suggesting fusion of both types of QRS-configuration were frequently seen before the original tachycardia recurred (Fig. 2, panel 3).

Apparently the original tachycardia persisted during the rhythm induced by pacing.

Resumption of the original tachycardia can be explained by a higher intrinsic rate of the original tachycardia (compared to the tachycardia induced by pacing). This is suggested by the events in Figure 1, panell, which shows that transition to the original tachycardia occurs with acceleration of ventricular rate. One can not exclude the possibility, however, that the pacing-induced ectopic activity persists for only a few beats and disappears because it rapidly becomes subthreshold. 


\section{The first postpacing interval}

Electrical stimulation during digitalis-induced ventricular tachycardias produces characteristic changes in the first postpacing interval.

It is not clear why a greater amount of shortening of the first postpacing interval could be induced during the single and double extrastimulus experiments than during the overdrive experiment and overdrive extrastimulus experiments.

Apparently overdrive stimulation had some depressant effect on the first postpacing interval.

From these observations we conclude that during digitalis-induced ventricular tachycardias, pacing results in a postpacing changes dependent on the site of pacing, and the last paced interval. The first postpacing interval is longer than the mean tachycardia interval after long extrastimulus intervals, and shortens with increasing extrastimulus prematurity. The duration of the first postpacing interval is composed of the conduction time from the site of stimulation to the site of impulsformation and the resetted discharge interval of the postpacing tachycardia focus. This might explain the pause that is greater than the tachycardia cycle length at long coupling intervals.

Acceleration of the first postpacing complex after an increase in pacing rate has also been observed in studies in the intact heart at lower digitalis levels, in the stage without overt ectopic ventricular activity $74,160,161$. The length of the first postpacing interval appeared to be dependent on the rate of the preceding test stimuli ${ }^{160,161}$ and the degree of digitalis intoxication ${ }^{160}$. Hogan et al. ${ }^{57}$ showed a similar behaviour of the first postpacing interval in the isolated Purkinje fiber. Zipes et al. ${ }^{161}$ showed an additional shortening effect of premature ventricular stimuli after constant ventricular pacing, whereas the reverse was observed after postmature extrastimuli. These authors ${ }^{161}$ also showed that constant atrial pacing at increasing rates followed by a ventricular extrastimulus at a fixed interval resulted in shortening of the first postpacing interval.

In our study we have systematically analyzed the effect of different modes of pacing during sustained ventricular tachycardia. We have found, that the first postpacing interval is determined principally by the last pacing interval. Further work needs to be done to investigate this behaviour in relation to different levels of digitalis intoxication.

\section{Effect of catecholamines on the behaviour of the first postpacing interval.}

Electrical stimulation is known to release stored catecholamines from nerve elements within the myocardium ${ }^{133}$, and catecholamines may facilitate digitalis-induced arrhythmias ${ }^{6,31}$. One has to consider the possibility that release of catecholamines by pacing may lead to acceleration of the postpacing interval. Although adrenergic blockade was not used during our experiments, we believe that this possibility can be ruled out by the observation that single and double stimuli resulted in a more marked shortening of the first postpacing interval than overdrive pacing. Furthermore, none of the dogs exhibited evidence of hemodynamic embarrassment during the programmed stimulation studies. Thus, the probability that increased sympathetic tone accounted for shortening of postpacing intervals is small.

We also noted that during the overdrive extrastimulus studies the postpacing interval returned to its initial value when a premature stimulus was applied within the absolute refractory period of the ventricle. This indicates that the non-followed stimulus had no influence on the first postpacing interval. 
This is in agreement with observations by other investigators ${ }^{\text {"4 }}$, who could exclude an influence of norepinephrine release from nerve terminals by electrical stimulation.

\section{Role of site of stimulation in the configuration of the first postpacing complex.}

Of great interest are our observations on the configuration of the first QRS-complex after pacing. The higher sensitivity of Purkinje tissue for digitalis glycosides in comparison with ventricular muscle has been documented by several authors ${ }^{32,1.19}$. Our present findings seem to support these observations. Foci close to the stimulation site can become temporarily manifest after pacing (Fig. 1,2 and 3). The configuration of the paced complexes differed from the postpacing complexes, in that paced complexes had a slow initial deflection, whereas postpacing complexes had a rapid initial deflection. This suggests that during pacing, slow intramuscular conduction is followed by invasion of nearby Purkinje fibers, triggering activity in these fibers leading to postpacing complexes with a rapid initial deflection in the QRS-complex.

Pacing at two different sites in the ventricles resulted in differences in QRS-configuration with a similar behaviour of the first postpacing interval. These observations suggest that during digitalis-induced ventricular tachycardia, a dominant focus gives rise to the spontaneous ventricular tachycardia, but other ventricular foci can temporarily be triggered by pacing. These different foci show essentially identical electrophysiological properties (Fig. 3). Other authors have also found that escape beats after pacing consistently originated in the stimulated ventricle ${ }^{16 !}$.

The most likely explanation for this is that a potential focus within the conduction system, close to the site of stimulation, will be discharged earlier than the dominant (more distant) focus ${ }^{161}$. The morphologic similarities between the paced complexes and the QRScomplexes after pacing suggest an origin of the triggered complexes close to the stimulation site. This suggests that in digitalis toxicity, many parts of the peripheral Purkinje system can be triggered into activity. Our observation that the length of the first postpacing interval is equal, independent of the site of stimulation is an attractive explanation for the clinically observed phenomenon in digitalis-induced arrhythmias of polymorphic ventricular ectopic activity having the same coupling interval ${ }^{118}$. It is intriguing, that during pacing far from the site of sustained abnormal impulse formation, we could never induce a sustained ventricular tachycardia with the same configuration as that of the first postpacing QRS-complex. This suggests that a dominant rhythm arising in the Purkinje system existed during digitalis intoxication and regained control of the heart immediately after pacing. This is supported by several reports ${ }^{19,71,94}$ showing the presence of predilection sites for impulse formation within the Purkinje system during digitalis intoxication.

\section{Mechanism of digitalis induced ventricular tachycardia.}

Theoretically one has to consider that digitalis-induced ventricular tachycardia can be based on reentry, delayed afterdepolarizations or abnormal automaticity. In ventricular tachycardias caused by reentry frequently an inverse relation is found between the prematurity of the tachycardia-initiating beat and the first beat of the tachycardia after 
pacing ${ }^{142}$. In our experiments, the tachycardia configuration remained unchanged when pacing was performed close to the origin of the tachycardia. Pacing at "distant" sites temporarily suppressed dominant tachycardia, and tachycardias arising close to the site of stimulation became manifest. In all our experiments, the first postpacing interval decreased as the last paced interval decreased. This is in contrast to what is frequently observed in reentry, and therefore makes reentry an unlikely mechanism for digitalis induced arrhythmid.

Another mechanism for digitalis-induced ventricular arrhythmias is triggered activity based on delayed afterdepolarizatoins. These afterdepolarizations occur after repolarization of action potentials ${ }^{17,18,32,35,48,97}$ and appear as oscillations of the diastolic membrane potential.

When an afterdepolarization reaches threshold, an action potential may be triggered. Coupling intervals and amplitudes of afterdepolarizations are dependent on the number of beats and the rate of the preceding rhythm and on the degree of digitalis intoxication. Premature stimuli after pacing at a constant rate havant rate have an additional accelerating effect on the coupling intervals. Long trains of spontaneous activity can be triggered under appropriate circumstances. If one considers afterdepolarizations as the mechanism of ectopic activity after pacing, one would expect the first postpacing interval to be dependent on the rate of overdrive pacing during the overdrive extrastimulus study. This was not observed during our experiments.

The results from our study, however, are difficult to compare with previous observations, because we continued administration of digoxin until sustained ventricular tachycardia occurred. It is of interest that at very long extrastimulus coupling intervals, the first postpacing interval was not short compared with the mean tachycardia interval. However, shortening of the extrastimulus coupling interval resulted in shortening to the first postpacing cycle (to the mean tachycardia cycle length) and ultimate "acceleration" after very short extrastimulus coupling intervals.

Abnormal automaticity can occur in Purkinje fibers in which phase 4 depolarization arises from a reduced maximum diastolic potential ${ }^{54}$. It has been shown that a decrease in the maximal diastolic potential occurs during digitalis toxicity ${ }^{57,86,98,99}$. After pacing, phase 4 depolarization appears to be suppressed during digitalis administration ${ }^{32,99}$, explaining the decrease in idioventricular rate after digitalis intoxication in the intact dog heart ${ }^{126,160}$. Rosen and Danilo ${ }^{99}$ also observed an increase in the slope of plase 4 depolarization in a minority of their experiments in isolated Purkinje fibers. These fibers had undergone a greater loss of membrane potential than the ones in which suppression of phase 4 depolarization had occurred. After overdrive pacing, they could induce acceleration of the first postpacing interval by increasing the rate of pacing. Possibly this acceleration was due to enhanced phase 4 depolarization.

The behaviour of the postpacing interval of the ventricular tachycardia after overdrive pacing in our study can be partially explained by the obserwations of Rosen and Danillo $\%$. However, we found that the duration of the first postpacing interval, is determined by the last paced interval and that acceleration can be induced by a single extrastimulus. We are not aware of any experimental data in isolated Purkinje fiber studies, which explains our findings.

It is possible that the behaviour of Purkinje fibers is different during sustained tachycardia compared with the situation in which manifest action potentials are triggered only by pacing. 


\section{Possible clinical implications}

Our observations show that in severe digitalis intoxication, the last paced beat determines the first postpacing interval.

Acceleration of this interval can be observed by giving a single premature beat with a sufficiently short interval. We have also seen that the pacing site influences the configuration and the site of origin of the first postpaced beat, although the dominant arrhythmogenic mechanism is likely to regain control after a few cycles. These data are of interest when the electrocardiographic manifestations of ventricular arrhythmias in human digitalis intoxication are considered. Future work is required to establish their value in improving our ability to diagnose digitalis intoxication in the human heart. 


\section{Effect of programmed electrical stimulation on the first postpacing QRS-interval during digoxin intoxication.}

by

Anton PM Gorgels, MD, Bram de Wit, MD, Henriete DM Beekman, Willem RM Dassen, PhD, Hein JJ Wellens, MD Deparment of Cardiology Annadal Hospital MAASTRICHT The Netherlands

Accepted for publicarion in PACE

\section{Summary}

In isolated Purkinje fibers digitalis intoxication induces triggered activity. The role of digitalis in inducing triggered activity in the intact heart is presently not well known. Therefore the effect of digitalis intoxication in the intact heart was studied by programmed electrical stimulation.

The experiments were done in conscious dogs with chronic compllete atrioventricular block. Ventricular tachycardia was induced with digoxin i.v. $0.1 \mathrm{mg} / \mathrm{kg} / \mathrm{I}-\mathrm{I} / \mathrm{t} / 2 \mathrm{hr}$. The effect of programmed electrical stimulation on the first postpacing interval was determined during episodes of sustained and of non-sustained ventricular tachycardia and during the episode that ectopic activity could only be induced by pacing. During sustained ventricullar tachycardia there was a direct linear relation between the interstimulus interval of regular pacing and the first postpacing interval. During episodes of non-sustained ventricular tachycardia shortening of the interstimulus interval resulted in a biphasic behaviour of the first postpacing interval. Pacing intervals of more than $400 \mathrm{~ms}$ induced a first postpacing interval being equal to the interstimulus interval, whereas shorter pacing intervals induced a first postpacing interval being twice the pacing interval. When during regular pacing only the last pacing interval was changed a simillar biphasic response resulted. When toxicity had almost subsided ectopic activity could only be induced following short pacing intervals (200m320 ms). Again a direct linear relation was found between the pacing interval and the first postpacing interval. Our findings strongly suggest that at all levels of digitalis intoxication triggered activity is the underlying mechanism for the first postpacing QRS-complex.

This chapter reports on further observations on the effect of programmed electrical stimulation on the first postpacing QRS-interval.

As pointed out in the preceding chapter, programmed electrical stimulation during digitalis-induced ventricular tachycardia induces one or more QRS-complexes of a different configuration than the spontaneous tachycardia. During tachycardia, however, the pacing rate has to be high and the range of interstimulus intervals is small. Because we were interested to study the role of delayed afterdepolarizations in the occurrence of ventricular arrhythmias, it was necessary to study the induction of ectopic activity over a wider range of interstimulus intervals. Therefore programmed electrical stimulation was also done following spontaneous termination of ventricular tachycardia.

Our study protocol was based upon the characteristics of dalayed afterdepolarizations in digitalis intoxicated isolated Purkinje fibers $17,18,32,35,48,87,97,98,100,401$. 


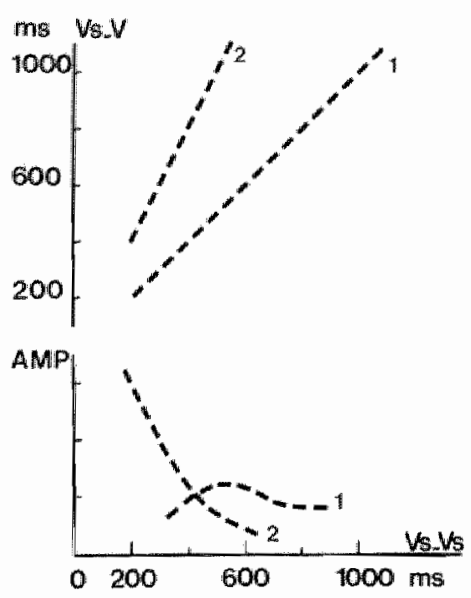

Figure 1.

Diagram modified from isolated Purkinje fiber sudies, relating the amplinde (lower panel) and coupling interval (upper panel) of delayed afterdepolarizations to the pacing interval.

Amp = amplitude

1 = first delayed afterdepolarizanion.

$2=$ second delayed afterdepolarization.

See text.

Delayed afterdepolarizations are oscillations of the diastolic membrane potential, occurring immediately after the repolarization phase of the action potential. When their amplitude is sufficiently high threshold is reached resulting in a full depolarization. After completion of this action potential again a delayed afterdepolarization occurs and possibly a full depolarization. In such a way long trains of spontaneous activity can be induced (triggered activity).

Enhanced phase 4 depolarization is also known to occur in digitalized Purkinje fibers ${ }^{100}$ and in orker situations, which induce partial depolarization of the diastolic membrane potential ${ }^{22}$ (abnormal automaticity).

In studies in isolated Purkinje fibers it has been found, that afterdepolarizations (DAD), inducing full depolarizations during digitalis toxicity, behave similarly as subthreshold delayed afterdepolarizations $\$ 7$. The relation of the coupling interval and the amplitude of DAD's to the interstimulus interval of the preceding rhythm is shown in figure 1 . This figure is modified from resu $\| \mathrm{t}$, obtained in isolated Purkinje fibers ${ }^{32,100}$. The upper panel shows the behaviour of the coupling interval $\left(V_{s}-V\right)$ of DAD's as a function of the interstimulus interval of the preceding rhythm $\left(V_{s}-V_{s}\right)$. The lower panel shows the height of the amplitude of DAD's in relation to the preceding interstimulus interval. The coupling interval of the first DAD shows a 1:1 relation to the pacing interval, and the coupling interval of the second DAD a 2:1 relation. The amplitude of the first DAD shows an increase on shortening the pacing interval, reaches a maximum between pacing intervals of 400 and $600 \mathrm{~ms}$, and declines on further shortening of the pacing interval. From these data the following characteristics of DAD induced ectopic activity are expected in the intact heart: 1) a direct linear relation between the interstimulus interval and the first postpacing interval;2) after short interstimulus intervals (less than $\pm 500 \mathrm{~ms}$ ) 
the first postpacing interval should be twice the pacing interval, because the amplitude of the first DAD is low and the amplitude of the second DAD is high; 3) At long pacing intervals (more than $\pm 500 \mathrm{~ms}$ ) the interval to the induced QRS-complex is equal to the stimulus interval, because the amplitude of the first DAD is high in this interval range. At low levels of digitalis intoxication threshold is expected to be attained following short interstimulus interval, because the amplitude is highest in this interval range.

Based on these characteristics, we studied the first postpacing interval of ectopic activity induced by programmed electrical stimulation 1) during sustained ventricular tachycardia 2) following termination of ventricular tachycardia during episodes that ectopic impulse formation could be induced over a wide range of interstimulus intervals 3 ) during the episode following termination of spontaneous ventricular tachycardia that ectopic impulse formation could only be induced by pacing with short interstimulus intervals.

\section{Methods}

The experiments were performed in mongrel dogs of either sex with a body weight between 20 and $40 \mathrm{~kg}$.

Through a right thoracotomy electrodes were fixed intramurally into the free wall of the right ventricle and the apex of the left ventricle. Atrioventricular block was made by injecting formalin $37 \%$ in the region of the His bundle ${ }^{114}$. The experiments were performed after a recovery period of at least one week. This period of rest was necessary to avoid episodes of spontaneous ventricular tachycardia during the experiment which are known to occur during the early postoperative period ${ }^{59,128}$. Six surface electrocardiographic leads (I, II, III, AVR, AVL and $V_{1}$ ) were simultaneously displayed on a monitor, registered with an ink jet recorder (with a paper speed of $100 \mathrm{~mm} / \mathrm{s}$ ) and stored on tape. Electrical stimulation was done with a programmable stimulator, having a synchronizing circuit. Unipolar stimuli were given with a stimulus strength of twice the diastolic threshold. Between consecutive stimulation trains enough time was allowed for the ventricular rhythm to regain its prepacing rate and QRS-configuration.

Digitalis intoxication was induced by an intravenous infusion of Digoxin in a dose of 0.1 $\mathrm{mg} / \mathrm{kg}$ during $1-1 \frac{112}{\mathrm{hr}}$. The pacing protocol included overdrive stimulation, varying the interstimulus interval and number of basic stimuli. In each stimulation train the interstimulus interval was shortened in steps of 10 or $20 \mathrm{~ms}$, starting with the longest possible interstimulus interval. Minimal values were $150 \mathrm{~ms}$.

The minimal number of stimuli given was one. After the diastole was scanned with increasingly shorter pacing intervals, the number of stimuli was increased and the stimulation procedure was repeated.

\section{Results}

The total amount of digoxin given in 8 dogs varied between 0,10 and $0,15 \mathrm{mg} / \mathrm{kg}$. This resulted in sustained ventricular tachycardia in 5 dogs. The mean interval duration of the tachycardias induced varied between 277 and $476 \mathrm{~ms}$ (table 1 ).

When the ventricular tachycardia stopped spontaneously, it was possible to induce ectopic impulse formation in three dogs over a wide range of interstimulus intervals (200-800 ms). When digitalis toxicity dissipated further ectopic impulse formation could only be induced following short interstimulus intervals. In 7 dogs this latter episode could be studied by programmed electrical stimulation. 


\begin{tabular}{lllll} 
dog & weight(kg) & dose mglkg & $V_{a}-V_{o}(m s)$ & $S D(m s)$ \\
\hline 1 & 27 & 0,14 & 277 & 16 \\
2 & 25 & 0,14 & 458 & 19 \\
3 & 25 & 0,10 & 334 & 14 \\
4 & 32 & 0,15 & 298 & 22 \\
5 & 40 & 0,11 & 476 & 93 \\
\hline
\end{tabular}

$\mathrm{SD}=$ standard dewiation.

\section{The effect of programmed electrical stimulation}

Overdrive stimulation of spontaneous sustained ventricular tachycardia resulted in a similar behaviour of the first postpacing QRS-complex as described in the preceding chapter.

One or more QRS-complexes with a different configuration were induced after which the original tachycardia regained control over the heart. The configuration of these induced QRS-complexes will be discussed in the next chapter.

Interstimulus intervals being only slightly shorter than the cycle length of the spontaneous tachycardia resulted in a first postpacing interval which was $150-200 \%$ of the interstimulus interval duration. Shortening of the interstimulus interval decreased the first postpacing interval. This resulted in a concordant relation between the interstimulus interval and the first postpacing interval. As expected from the results from the preceding chapter, the duration of the first postpacing interval was relatively independent from the number of stimuli. This is shown in figure 2 in which the relation between the interstimulus interval and the first postpacing interval for different numbers of stimuli are given from five experiments in different dogs.

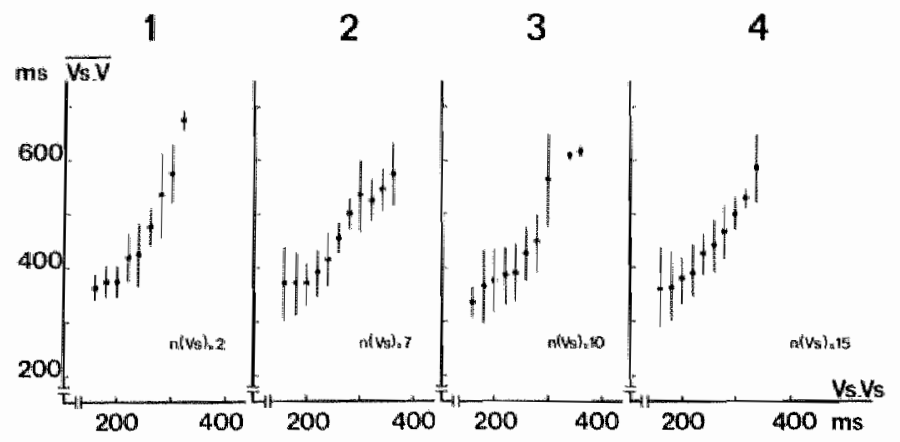

Figure 2

The influence of the interstimulus interval on the first postpacing interval during sustained ventricular tachycardia.

Combined results of 5 experiments in different dogs. On decreasing the pacing interval the first postpacing intervat shortens. The duration of the first postpacing interval is about $150 \%$ of the pacing cycle length. 
After spontaneous termination of the tachycardia ectopic impulse formation could be reinduced by pacing over a wide range of cycle lengths and different numbers of stimuli in three dogs. In a minority of runs $(20 \%)$ no ectopic activity occurred. When the toxicity level was sufficiently high pacing resulted in ventricular tachycardia (figure 3). At lower degrees of toxicity only short self-terminating runs of ectopic activity were induced. When pacing with long intervals (more than $\pm 400 \mathrm{~ms}$ ), ectopic activity started frequently before the stimulation train was completed. This occurred because the escape interval of the induced rhythm was about equal to the pacing cycle length. Using shorter interstimulus intervals the first postpacing interval was longer than the interstimulus interval.
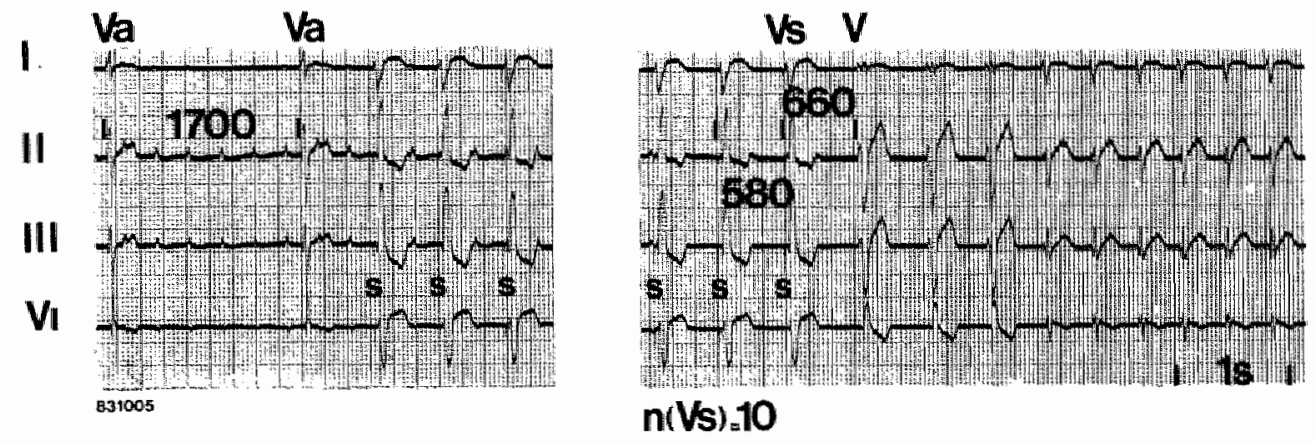

Figure 3

Induction of ectopic activity by pacing during slow idioventricular rhythm.

10 stimuli with interstimulus intervals of $580 \mathrm{~ms}$ are given. The first postpacing interval is within the range of the intersitimulus interval. Acceleration occurs in the next wo intervals, which are followed by a tachycardia with a different configuration.

Examples of induction of ectopic impulse formation by fast and by slow pacing are given in figure 4.

When the interstimulus interval was decreased systematically it was found that after long interstimulus intervals (more than $\pm 400 \mathrm{~ms}$ ) the first postpacing QRS-complexes had an interval duration being in the same range as the interstimulus interval.

Shortening the interstimulus interval to a critical value induced a sudden lengthening of the first postpacing interval. On further shortening of the pacing interval a similar relation with the first postpacing interval was obtained as observed during sustained ventricular tachycardia. To illustrate these findings an example of pacing with 4 stimuli is given in figure 5. 


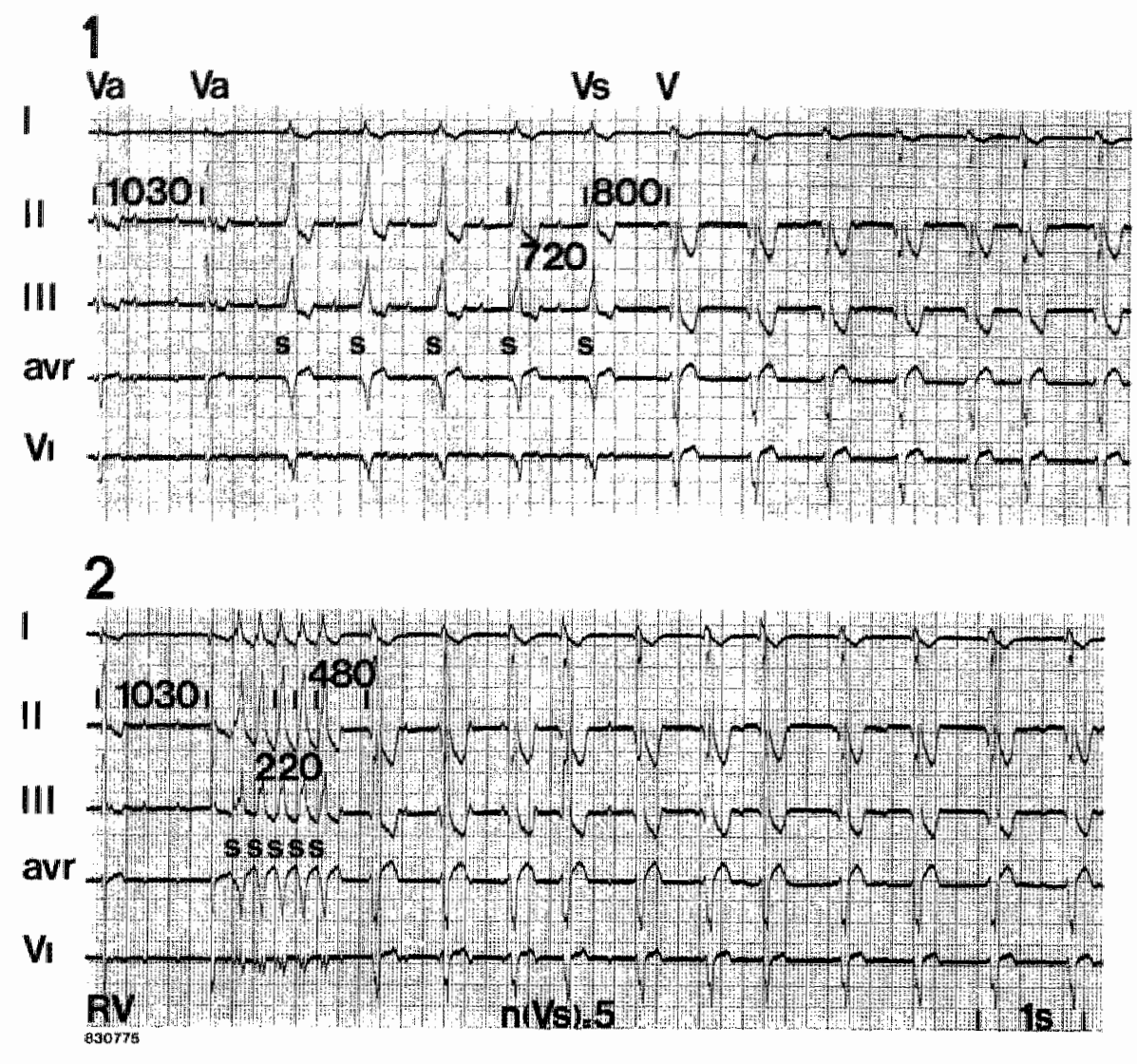

Pigure 4

Induction of ectopic activity by slow (panel i) and fast pacing (panel z).

The diowentriculat rhythm is overpaced with s stimuli. Slow pacing $(720 \mathrm{~ms}$ ) results in ectopic activity. The Virst postpacing interval is within the range of the drive cycle length.

Pacinew wh $220 \mathrm{~ms}$ interwals results in similar ectopic acivity as in panel 1 . Now the first postpacing interval is about twise the drive cycle length.

From panel 1 to 3 the first postpacing interval has a $1: 1$ relation to the pacing interval. Berween interstimulus intervals of 440 and $350 \mathrm{~ms}$ no ectopic activity could be induced. Following interstimulus interyals of $350 \mathrm{~ms}$ the first postpacing interval showed an abrupt lengthening in comparison to the preceding panel. Further shortening of the interstimulus interval again resulted in a decrease of the first postpacing interval.

Figure 6 shows diagrammatically this biphasic behaviour for all first intervals of induced ectopic activity.

Simillar results were obtained following different numbers of stimuli. The results from three different experiments are combined in figure 7 . The curves indicating a 1: 1 relation and the one indicating a 1:2 relation show some overlap because the interval shift occurred at different interstimulus intervals in different dogs. 

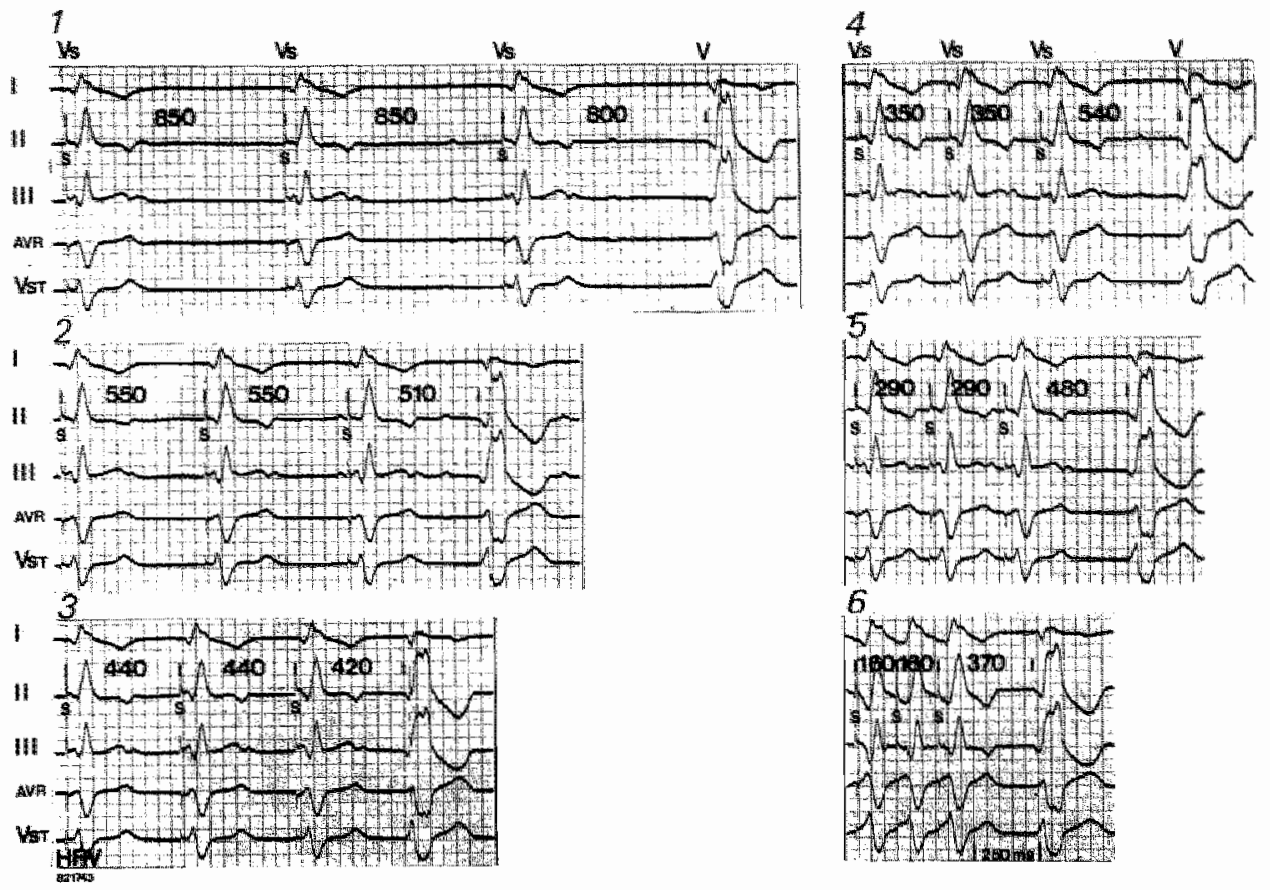

Figures 5

The influence of the interstimulus interval during non-sustained ventricular tachycardia.

Example of overdrive pacing with four stimuli at the basal right ventricle. Only the last three stimulated QRS-complexes and the first postpacing QRS-complex are shown. Four standard leads and a chest lead $\left(\mathrm{V}_{\mathrm{s}}\right)$ are displayed simultaneously. From panel 1 to panel 6 the interstimulus interval decreases from 850 to $160 \mathrm{~ms}$. See text.

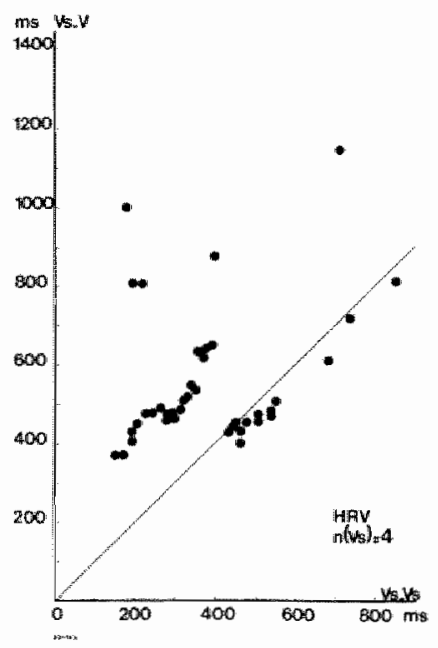

Figure 6

Diagram, showing all first postpacing intervals from the experiment in figure 5 . Note the biphasic behaviour of the first postpacing interval on decreasing the pacing interval. 


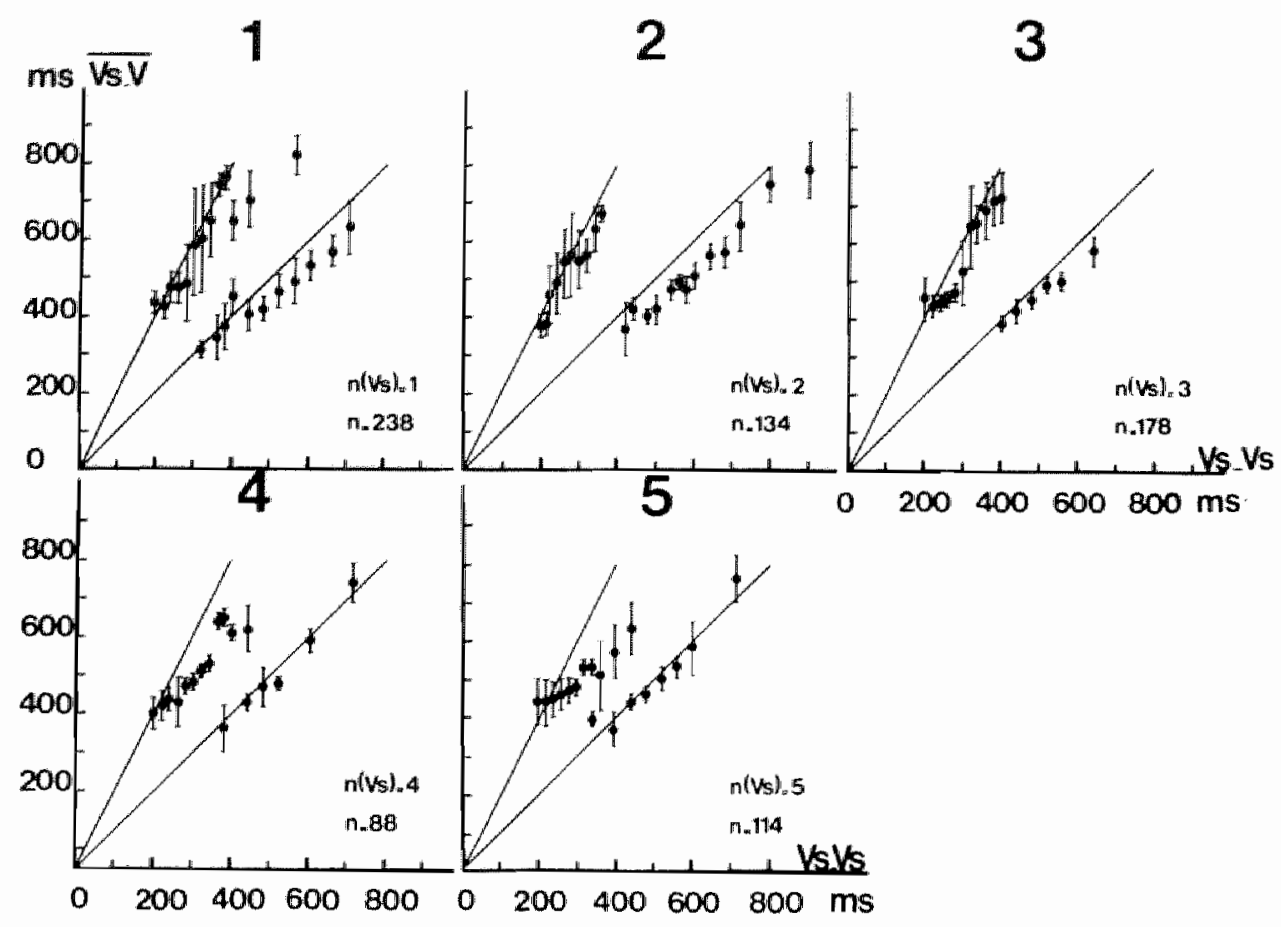

Figure 7

The influence of the interstimulus interval on the first postpacing interval at different numbers of stimuli during non-sustained ventricular tachycardia.

Combined data from 3 experiments. The first postpacing interval (mean and standard deviation) is plotted as a function of the first postpacing interval.

From panel 1 to panel 5 the number of stimuli increase from 1 to 5 . The number of stimulation urains ( $\mathrm{n}$ ) is indicated in esch panel. Lines indicating a $1: 1$ relation and a $1: 2$ relation between the interstimulus inserval and the first postpating interval ate given in each panel.

The effect of extrastimuli following regular pacing was difficult to study because, as mentioned above, during regular pacing frequently the spontaneous rhythm started before the stimulation train was completed. In the different examples obtained it was found that decreasing the extrastimulus interval only, also resulted in a similar biphasic behaviour of the first postpacing interval as observed during regular pacing. An example is shown in figure 8. 


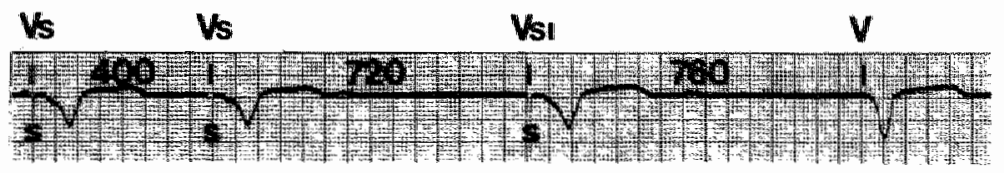

\section{2}

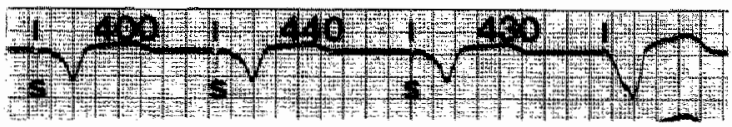

3
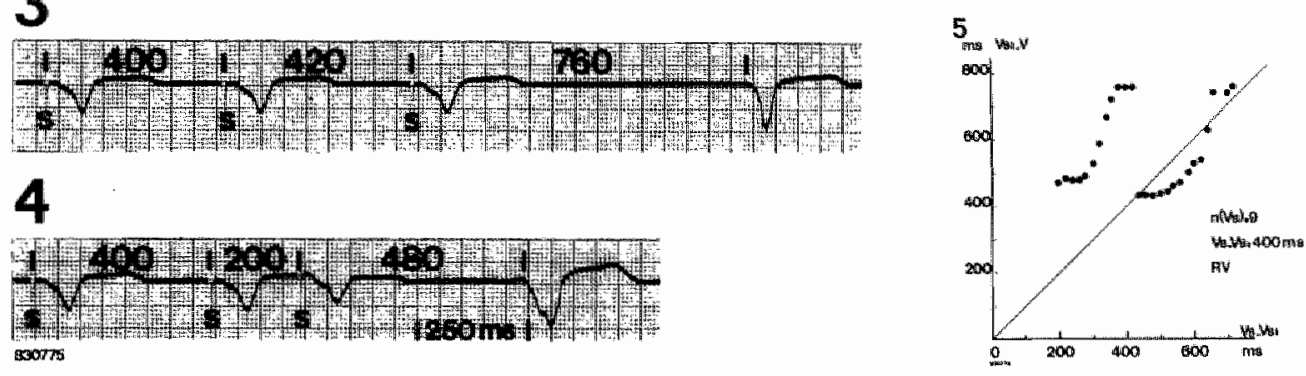

Figure 8

The effect of shortening the extrastimulus interval on the first postpacing interval during non-sustained ventricular tachycardia.

Basic pacing is done with 9 stimuli and pacing intervals of 400 ms. The extrastimulus interwal decreases from 720 to $200 \mathrm{~ms}$ (panel 1 to 4). Only the last three stimulated complexes are shown.

\section{Observations at the time of cessation of digitalis intoxication}

During this episode only short runs of ectopic activity were indwced by overdrive stimulation. For induction of ectopic activity short interstimulus intervals and higher number of stimuli were required. Overdrive suppression occurred between pacing intervals of 1000 and $500 \mathrm{~ms}$. At a critical pacing rate the first postpacing interval shortened abruptly to about twice the pacing interval. Decreasing the interstimulus interval further resulted in a further decrease of the first postpacing interval. Examples are given in figure 9 and 10.

Figure 11 shows the combined data (mean and standard deviation) of 7 other experiments during pacing with 10 stimuli at the end stage of digitalis intoxication. Ectopic activity could be induced in all experiments using interstimulus intervals between 200 and 320 ms. At longer pacing intervals overdrive suppression was observed. 
1

$v_{1}$

Va Va

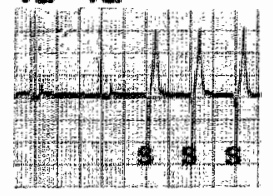

\section{2}

v

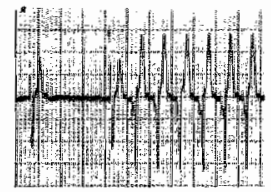

3

$\mathbf{v}_{\mathbf{1}}$

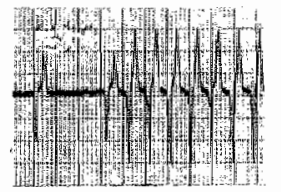

\section{4}

$\mathbf{V}_{\mathbf{I}}$

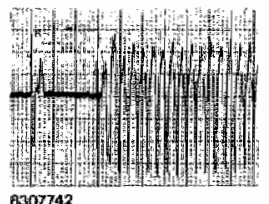

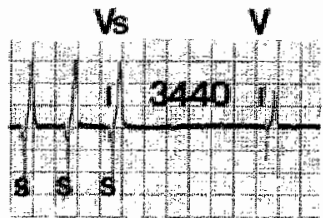

Vs.Vs.1000ms

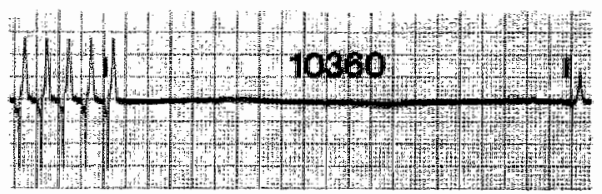

$\mathrm{Vs}_{\mathrm{s}} \mathrm{Vs}_{\mathrm{s}} 500 \mathrm{~ms}$

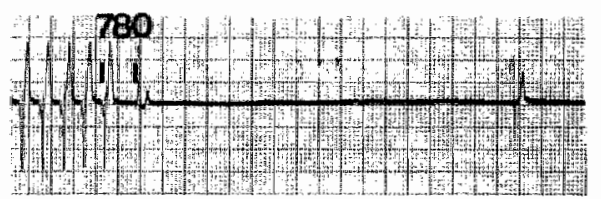

Vs.Vs.470ms

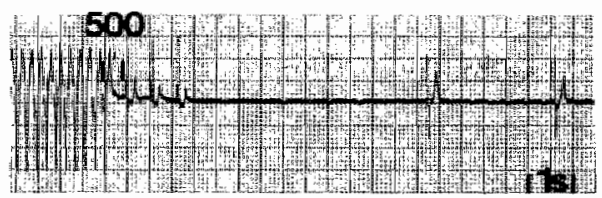

$V s=220 \mathrm{~ms}$
$n(V s)_{m} 50$

Figuric: 9

The influence of the interstimulus interval on the first postpacing interval at the end of digitalis intoxication. Dizumple of pacing with 50 stinuli. Fach panel shows the last prepacing interval, part of the pacing procedure and the interval(s) after pacing. From pand 1 to pand 4 the inferstimulus interval shortens from $100010220 \mathrm{~ms}$. Decreasing the pacing interval from 1000 to 500 mas results in marked overdrive suppression (panel 1 and 2 ). A small further decrease $10470 \mathrm{~ms}$ induces ectopic activity (panel 3 ) after $780 \mathrm{~ms}$. In panel the number of ectopic impulse increases and the interval of the first postpacing interval shortens to $500 \mathrm{~ms}$.

Sece text. 


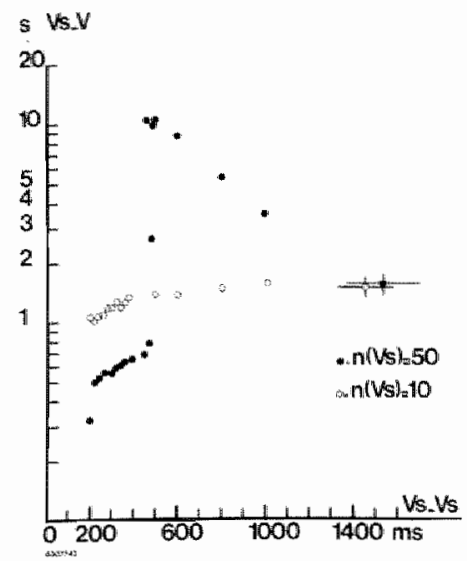

Figure 10

Influence of the interstimulus interval and the number of stimuli on the first postpacing interval during the end stage of digitalis intoxication.

The first postpacing interval is plotted on a logarithmic scale. Black dots indicate the first postpacing interval following 50 stimuli and open dors following 10 stimuli. The mean and standard deviation of the last prepacing interval is indicated at the right side. 10 stimuli induce only small changes of the first postpacing interval but no ectopic activity.

Overdrive suppression occurs after pacing with 50 stimuli between pacing intervals of 1000 and $500 \mathrm{~ms}$. Furoher shortentag of the pacing interval leads to a short first postpacing interval (about twice the pacing interval) with a direct linear relation to the pacing interval.

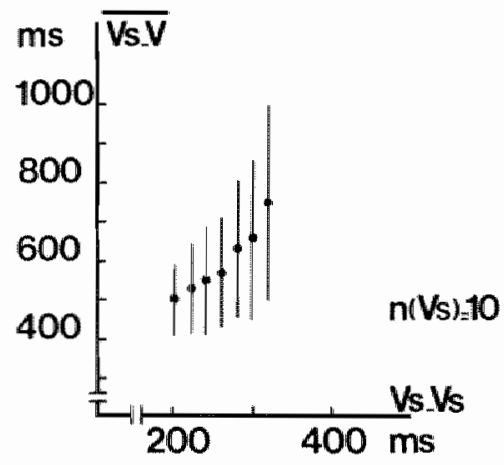

Figure 11

The influence of the interstimulus interval on the first postpacing interval at the end of digitalis toxicity. Combined results of 7 experiments. Ectopic activity is only inducible berween pacing intervals of 200 and 320 ms. The first postpacing interval (mean and standard deviation) is depicted as a function of the pacing interwal. 


\section{Discussion}

During sustained ventricular tachycardia we observed a direct linear relation between the first postpacing interval of the induced ectopic activity and the interstimulus interval. The duration of the first postpacing interval was $150-200 \%$ the interstimulus interval. The influence of the interstimulus interval was of more importance than the number of stimuli in determining the duration of the first postpacing interval (figure 2). These results were expected from the data presented in the preceding chapter.

During episodes of non-sustained ventricular tachycardia the relation between the interstimulus interval and the first postpacing interval was biphasic i.e. long interstimulus intervals showed a 1:1 relation to the first postpacing interval, whereas the first postpacing intervals following shorter interstimulus intervals had a duration of about $2 \times$ the interstimulus interval. In the experiment of figure 5 no ectopic activity was induced between interstimulus intervals of 440 and $350 \mathrm{~ms}$. The most likely explanation for this Inding is that both delayed afterdepolarizations were low in this interval range ${ }^{100}$. At low levels of digitalis intoxication the induction of ectopic impulse formation depended on the duration of the interstimulus interval and number of stimuli.

These findings are compatible with the described characteristics of delayed afterdepolarizations.

Because during sustained ventricular tachycardia the relation between the pacing interval and the first postpacing interval was similar, it is likely that also the first postpacing interval during ventricular tachycardia is the result of the (second) delayed afterdepolarization. The fact that the interval duration of the first postpacing interval was less than twice the pacing interval can be explained by assuming that at these severe intoxication levels threshold is attained before the amplitude becomes maximal.

In the preceding chapter it was shown, that during ventricular tachycardia shortening of the extrastimulus interval only, already resulted in a decrease of the first postpacing interval. During the episode that ectopic activity could be induced over a wide range of cycle lengths, shortening of the extrastimulus interval resulted in a similar biphasic behaviour of the first postpacing interval as observed following regular pacing. These findings are similar to the observations in isolated Purkinje fiber studies on the behaviour of delayed afterdepolarizations ${ }^{32,87}$.

Another explanation for the biphasic response to changing the interstimulus interval has to be considered. In sucrose-gap preparations sub-and suprathreshold depolarizations have been found to modulate Purkinje fiber pacemakers in a similar biphasic manner as observed in our experiments ${ }^{62}$. In these experiments sub-or suprathreshold depolarizing currents were applied distal to the pacemaker site. Conduction block was achieved through a sucrose-gap between both sites in the Purkinje fiber preparation.

Depolarizations, applied early in the cycle, delayed the subsequent pacemaker discharge, but when they were induced late in the cycle the pacemaker was captured. This biphasic influence was the result of the electrotonic effects of the depolarizations across the sucrose-gap. It is conceivable that digitalis intoxication also induced conduction block ${ }^{105}$ leading to similar conditions as described in these experiments.

The differences with the results of our experiments are, however, that we did not modulate the spontaneous site of impulse formation, but rather activated another pacemaker site. Also, when electrotonic modulation would have played a role, the 
biphasic response should have also been observed during electrical stimulation of the ventricular tachycardia. Finally, we have observed that subthreshold stimulation did not influence configuration and rate of digitalis-induced ventricular arrhythmias (not shown). Therefore it is not likely that electrotonic modulation is the underlying mechanism for the results of our experiments.

In conclusion our observations support the hypothesis that delayed afterdepolarizations are the underlying mechanism for the first postpacing QRS-complex at different tevels of digitalis intoxication.

\section{Clinical implications}

Patients with ventricular ectopic activity, ventricular tachycardia and/or atrioventricular block frequently confront the clinician with the possibility of digitalis intoxication as the underlying mechanism. Determination of the plasma level is of limited value, and one has to depend highly upon the clinical picture and the surface electrocardiogram ${ }^{118,140}$. In this situation programmed electrical stimulation of the heart might be of help in making the correct diagnosis. Such studies may also be of importance to obtain information on the effect of pharmacological interventions in the treatment of digitalis induced arrhythmias. It would be especially useful to be better informed about the value of drugs suppressing delayed afterdepolarizations like calcium antagonists, lidocaine and beta adrenergic blocking agents. 



\section{Chapter 5}

\section{Effect of different modes of stimulation on the Configuration of the first QRS-complex after pacing during digitalis induced ventricular tachycardia}

by

Anton PM Gorgels, MD, Bran de Wit, MD, Henriette DM Beekman, Willem RM Dassen, PhD, Hein J] Wellens, MD, Department of Cardiology Annadal Hospital MAASTRICHT The Netherlands

Accepted for publication in PACE

\section{Abstract}

During digitalis-induced sustained monomorphic ventricular tachycardia programmed electrical stimulation was done and the effect on the first postpacing QRS-configuration determined. Ventricular tachycardia was induced in 9 conscious dogs with chronic complete atrioventricular block by digoxin i.v. $0.1 \mathrm{mg} / \mathrm{kg}$ given in $\mathrm{x}-\mathrm{I} / 2$ hour. Spontaneous ventricular tachycardia had a right bundle branch block configuration and an extreme left axis in about $70 \%$ of tachycardias studied, suggesting an origin in the inferolateral part of the left ventricle. Less frequently a left bundle branch block like configuration with an intermediate axis was observed, compatible with an origin in the basal part of the right ventricle. After pacing close to one of these predilection sites the first postpacing QRS-configuration suggested an origin close to the site of stimulation. Pacing distant from these predilection sites resulted in fusion complexes between electrical activation from these predilection sites and the stimulation site. The amount of fusion depended on interstimulus interval and number of stimuli. Long interstimulus intervals and low numbers of stimuli induced a QRS-complex similar to the spontaneous tachycardia. The faster and longer the stimulation train the more the QRScomplex became similar to the paced QRS-complex. Similar findings were also found on decreasing the last paced interval only.

Our findings suggest that triggered activity is the underlying mechamism for the first postpacing QRS-complex. Analysis of the results of programmed electrical stimulation, and the relation between the R-R intervals and QRS-configuration during tachycardia suggest that triggered activity is also the mechanism for the spontaneously occurring ventricular tachycardia. These observations may have important clinical implications.

As has been shown in chapter 4 , in the intact heart the first QRS-complex after pacing is most likely to be induced by triggered activity $4,74,161$. We have found 42,43 a direct linear relation between the interstimulus interval and the first postpacing interval during sustained ventricular tachycardia. After termination of sustained ventricular tachycardia ectopic activity could be reinduced by pacing. Long interstimulus intervals (more than $t$ $400 \mathrm{~ms}$ ) resulted in a $\mathbb{1}: 1$ relation between the length of the first postpacing interval and 
the interstimulus interval while at shorter interstimulus intervals (less than $\pm 400 \mathrm{~ms}$ ) a $2: 1$ relation was observed. These findings are best explained by the rate related behaviour of the first two delayed afterdepolarizations ${ }^{87,100,101}$. The QRS-configuration of the spontaneous ectopic activity is frequently different from that during impulse formation immediately after pacing ${ }^{42}$ and has been found to originate preferably in the paced ventricle $^{i 61}$.

In the present chapter the relation between configuration of the first postpacing QRScomplex and modes of pacing during digitalis induced ventricular tachycardia is described. As discussed they have implications for the explanation of the possible underlying mechanism of the first postpacing QRS-complex and also the QRS configuration of the spontaneous ventricular tachycardia.

\section{Methods}

The experiments were performed in mongrel dogs of either sex with a body weight between 25 and $40 \mathrm{~kg}$.

Through a right thoracotomy electrodes were fixed intramurally into the free wall of the right ventricle and/or the apex of the left ventricle. Atrioventricular block was made by injecting formalin $37 \%$ in the region of the His bundle ${ }^{114}$. The experiments were performed after a recovery period of at least one week. This period of rest was necessary to avoid episodes of spontaneous ventricular tachycardia during the experiment which are known to occur during the early postoperative period ${ }^{59,128}$. Six surface electrocardiographic leads (mostly leads I, II, III, AVR, AVL and V ) and one epicardial lead were simultaneously registered on a ink jet recorder (with a paper speed of $100 \mathrm{~mm} / \mathrm{s}$ ) and stored on tape. Electrical stimulation was done with a programmable stimulator having a synchronizing circuit. Stimuli were given with a stimulus strength of twice the diastolic threshold. Between consecutive stimulation trains enough time was allowed for the ventricular rhythm to regain its prepacing rate and QRS-configuration.

Digitalis intoxication was induced by an intravenous infusion of digoxin in a dose of 0.1 $\mathrm{mg} / \mathrm{kg}$ which was given during $1-1 / 2$ hour. This resulted in sustained ventricular tachycardia during 12 to 48 hours.

All dogs were kept in the left lateral position to avoid influence on the QRS-configuration by changes in the position of the dog.

The first postpacing QRS-complex was analyzed only during episodes of sustained monomorphic ventricular tachycardia.

\section{Results}

During most episodes (about $70 \%$ of tachycardias analyzed) the spontaneous ventricular rhythm showed a right bundle branch block pattern with left axis deviation suggesting an origin in the apical part of the left ventricle. The second most frequent spontaneous QRS-configuration was a left bundle branch block pattern with an intermediate axis suggesting an origin in the basal part of the right ventricle (figure 1 ). Both configurations were frequently present during the same experiments. 

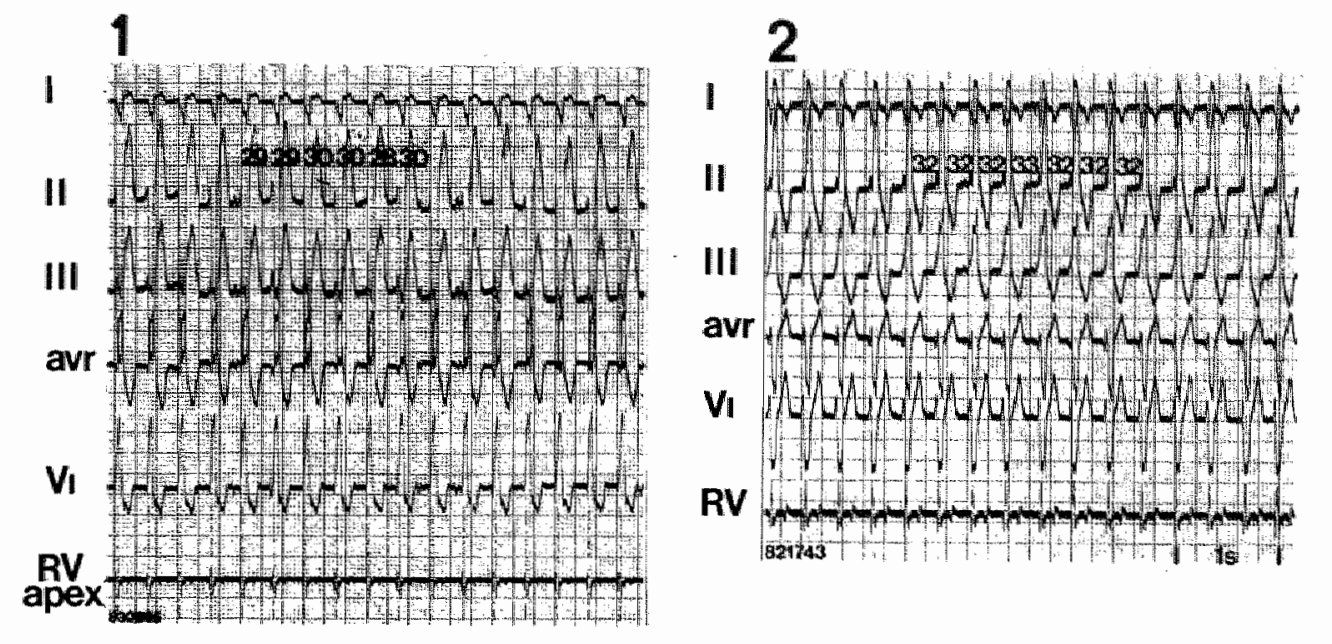

Figure 1

Typical examples of the QRS-configuration during digitalis induced ventricular tachycardia.

Left panel: tachycardia originating in the apical part of the left ventricle. Right panel: tachycardia originating in the basal part of the right ventricle.

\section{The effect of programmed electrical stimulation}

It was found that pacing close to one of these dominant pacemaker sites resulted in a first postpacing complex having the same QRS-configuration as these predilection sites.

Figure 2 shows left apical pacing during a right ventricular tachycardia. Pacing is done

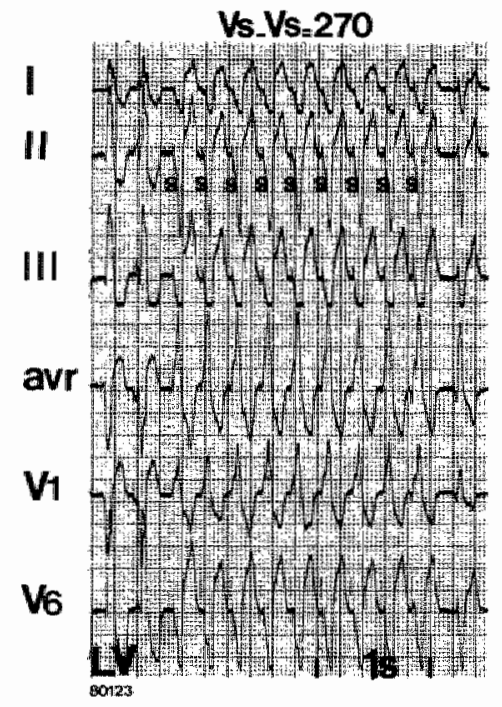

Figure 2
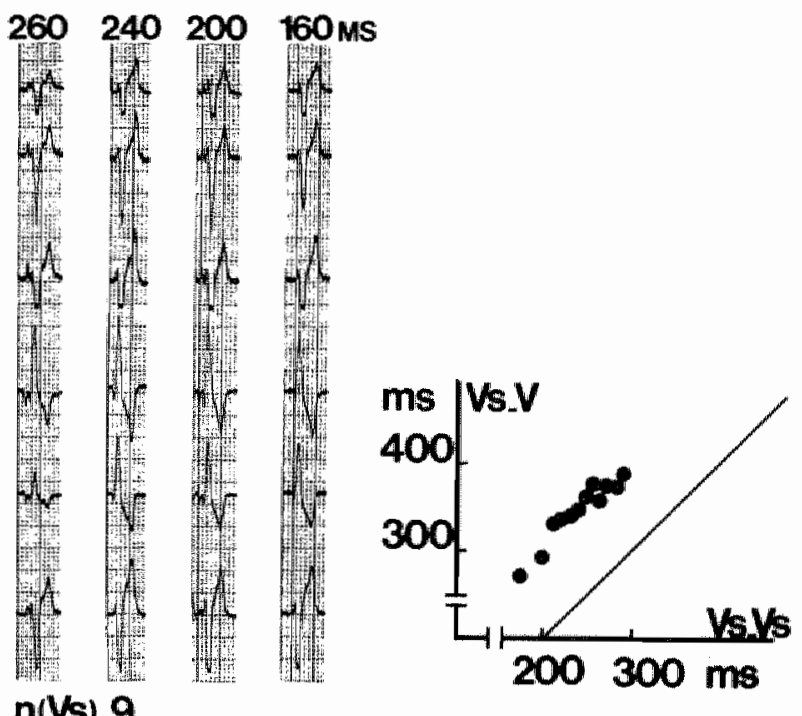

The effect of left apical stimulation during right ventricular tachycardia.

pacing is done with 9 simuli: In each stimulation train the pacing interval is shortened, resulting in an direct. linear relation between the pacing interval and the first postpacing interval (see diagram).

The QRS-configuration is very similar so the paced complexes after all stimulation trains (see texi). The small spikes preceding the QRS-complexes in panels 2 and 4 are P-waves. 
with a train of 9 stimuli at increasingly shorter interstimulus intervals. This results in a direct relation between the pacing interval and the first postpacing interval. The QRSconfiguration is very similar to the paced complexes after all stimulation trains and all originate in the apical part of the left ventricle. The only difference between the (epicardially) paced and the postpacing QRS-complex is the absence of the initial slurring of the QRS-complex in the postpaced beat. This suggests that during pacing ventricular activation starts in the muscle and in the post paced beat in the Purkinje system.

Pacing distant from both predilection sites resulted in a different first postpacing QRSconfiguration suggesting fusion between electrical activation from the predilection sites and also from the stimulation site. The amount of fusion appeared to be related to the length of the interstimulus interval.

Figure 3 shows the occurrence of fusion complexes between a focus in the basal right ventricle and the left ventricular apex. Overdrive stimulation at the apical part of the right ventricle is done during right ventricular tachycardia. Long interstimulus intervals induce a QRS-configuration similar to the spontaneous QRS-configuration. Shortening of the interstimulus interval results in fusion between this QRS-configuration and the QRS-configuration from the focus in the left ventricular apex. Decreasing the interstimulus interval results in an increasing contribution from left ventricular impulse formation.

Figure 4 presents diagrammatically the relation between the interstimulus interval and the first postpacing QRS-configuration of all stimulation trains of this experiment $(\mathrm{n}=$ 178). Interstimulus intervals of $420-320 \mathrm{~ms}$ induce predominantly a right ventricular configuration; between 320 and $220 \mathrm{~ms}$ most first postpacing QRS-complexes are fusion complexes; between 220 and $150 \mathrm{~ms}$ the left ventricular configuration prevails. These findings suggest that during pacing in between the two predilection sites for impulse formation both foci became activated. Because the stimulation site is closer to the left ventricular apex than to the site of origin of the spontaneous tachycardia, the contribution to ventricular activation of the former is the most important, especially at shorter interstimulus intervals.

We also observed the concomitant induction of impulse formation from the two dominant sites of spontaneous impulse formation and from the stimulation site.

This led to many fusion QRS-complexes showing gradual changes in configuration depending upon the interstimulus interval and the number of stimuli. In the experiment shown in figures 5-10 more than 40 different QRS-configurations could be identified. In this experiment overdrive stimulation was done by decreasing the interstimulus intervals in each stimulation train in steps of $10 \mathrm{~ms}$ to a minimum of $160 \mathrm{~ms}$. Thereafter the number of stimuli was increased and the procedure repeated. The number of stimuli was increased in steps of one stimulus from 1 to 10 and than to 15 stimuli. 


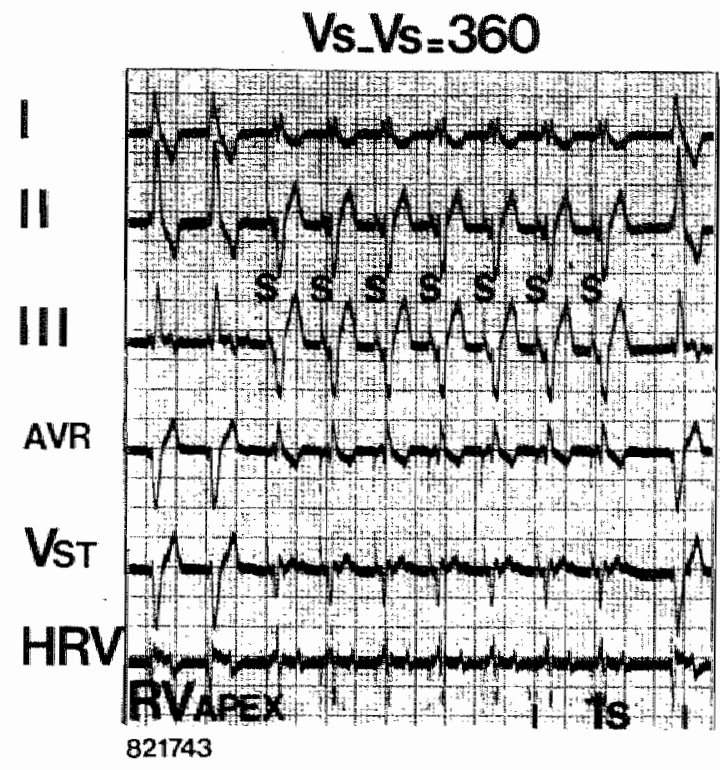

$310290240230170 \mathrm{MS}$

ms Vs.V

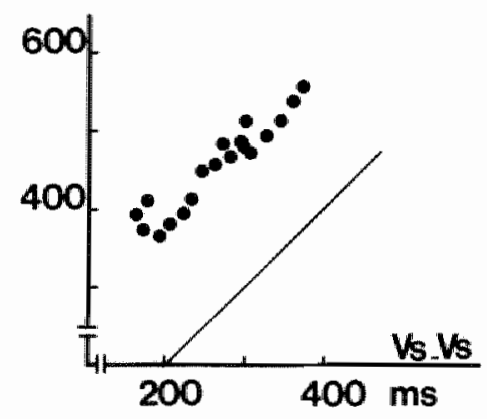

Figure 3

The effect of electrical stimulation distant to dominant pacemaker sites.

In this example the spontaneous ventricular tachycardia originates in the basal right ventricle (HRV, left panel). After pacing with 7 stimuli at the apical part of the right ventricle (RV-apex) and interstimulus intervals of $360 \mathrm{~ms}$ the first postpacing QRSconfiguration is unchanged (Ieft panel). Decreasing the in terstimulus inverval (right panels) results in a grad wal change of the QRS-complex singyesting in creasingly more fusion with impolse formation from the lefe wentriculat apex. The diagram shows a direce relation beween the pacing interval and the firsi post pacing interval.

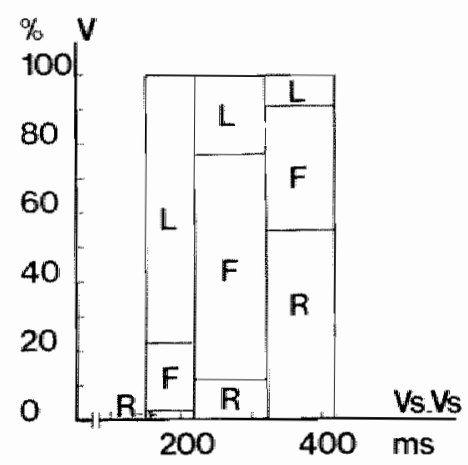

Figure 4

The effect of electrical stimulation distant to the doninant pacemaker sites.

Diagram showing the combined data from the experiment of ligure 3 using different numbers of stimuli. The rotal number of stimulation trains is 178 .

The percentage of QRS-complexes with a predominant feft ventricular origin $(\mathrm{L})$, right ventricular origin $(\mathrm{R})$, and fusion complexes (F) are plotted as a function of the intersimulus interwat. Note the predominance of righ ventricular QRS-complexes at longer interstimulus intervals, of fusion complexes at intermedote interstimulus intervals and of left ventricular QRS-complexes following short interstimulus intervals. 
In figure 5 during a left ventricular tachycardia overdrive stimulation is done with 9 stimuli from the mid-part of the free wall of the right ventricle. The diagram shows the direct linear relation between the interstimulus interval and the first postpacing interval. Long interstimulus intervals ( $260 \mathrm{~ms}$ ) induce a first postpacing QRS-configuration similar to the spontaneous QRS-complexes. Interstimullus intervals between 250 and 220 ms show a gradual change to a QRS-configuration suggesting an origin in the basal right ventricle. Decreasing the interstimulus interval further to $170 \mathrm{~ms}$ results in a change to a QRS-configuration compatible with an origin close to the stimulation site.
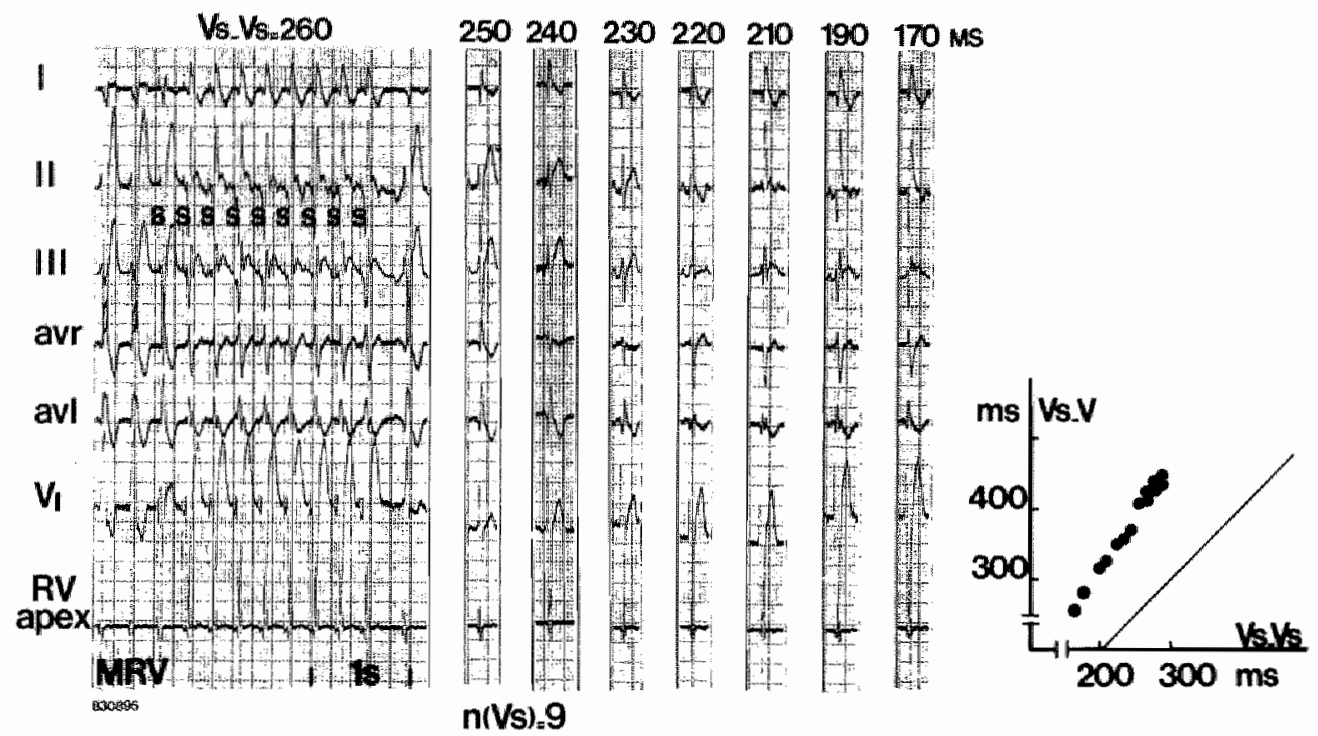

Figure 5

The influence of the pacing interval on the first postpacing QRS-morphology during electrical stimulation distant to dominant pacemaker sites.

The ventriculan tachycardia originates in the left ventricular apex. The ventricles are paced with 9 stimuli at the mid-portion of the right ventricle (MRV). Long interstimulus intervals (260 ms) induce a QRS-complex suggesting an origin in the apex of he left ventricle. Decreasing the interstimulus intervals $(250-220 \mathrm{~ms})$ results infusion between impulses from the left wentricular apex and the basal right ventricle. Further shortening leads to fusion between impulses fron the basal right ventricle and the stimulation site, until the first postpacing QRS-complex alosely resembles the stimulated QRS-complex ( $170 \mathrm{~ms})$.

From this experiment 1053 first postpacing QRS-complexes during sustained ventricular tachycardia using interstimulus intervals between 280 and 170 ms were analyzed. The QRS-configuration was divided in 5 groups from the one showing predominantly a left ventricular origin to the one showing an origin close to the stimulation site (see figure 6). The diagram (figure 6) shows the gradual change from a left apical origin after long interstimulus intervals to a predominantly right basal origin after intermediate interstimulus intervals and to an origin at the stimulation site after short interstimulus intervals. 
$M=6074 \quad 79$ as $\quad \begin{array}{llllllllll}100 & 94 & 104 & 135 & 156 & 111 & 38 & 17\end{array}$

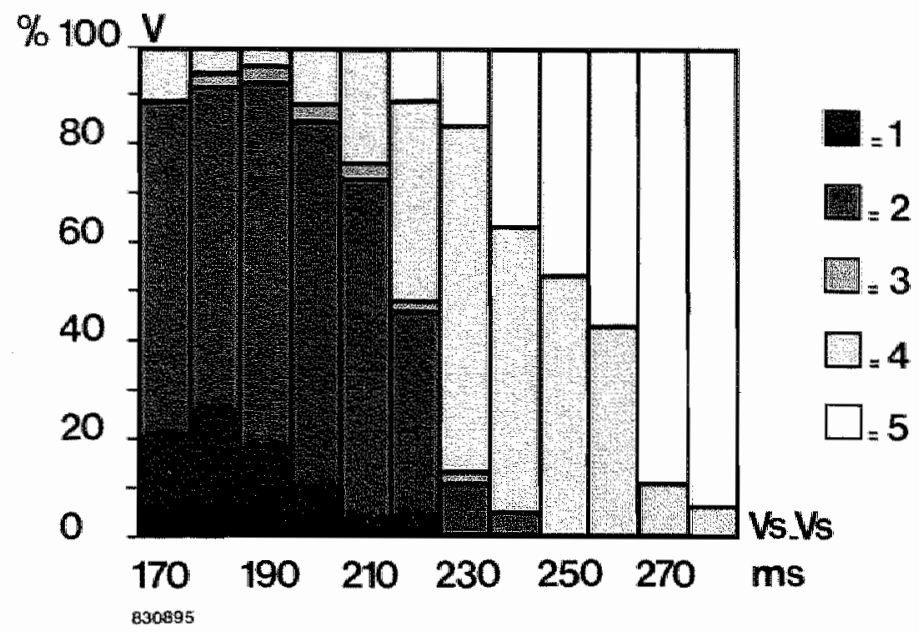

Figure 6

The influence of the pacing interval on the first postpacing QRS-morphology during electrical stimulation distant to dominant pacemaker sites.

Diagram relating the configuration of all QRS-complexes from the experiment of figure 5 to the preceding pacing interval (horizontal axis). The total number of QRS-complexes analyzed is 1053 . The bars indicate the percentage of occurrence of a given QRS-configuration.

1: a first postpacing QRS-configuration similar to the stimulated QRS-complexes.

2: fusion QRS-complexes between electrical activation fron the stimulation site and the basal right venuricle.

3: QRS-complexes from the basal right ventricle.

4: fusion complexes between electrical activation from the right and the left ventricle.

5: QRS-complexes from the left ventricle.

During this experiment the relation between the number of stimuli and the first postpacing QRS-configuration was also studied. Figure 7 illustrates the results. Increasing the number of stimuli from 1 to 5 induced a gradual change in QRSconfiguration from one suggestive of a left apical to a right basal origin. A further increase to 15 stimuli induced a QRS-configuration which resulted from fusion between a basal right and a mid right ventricular origin. 


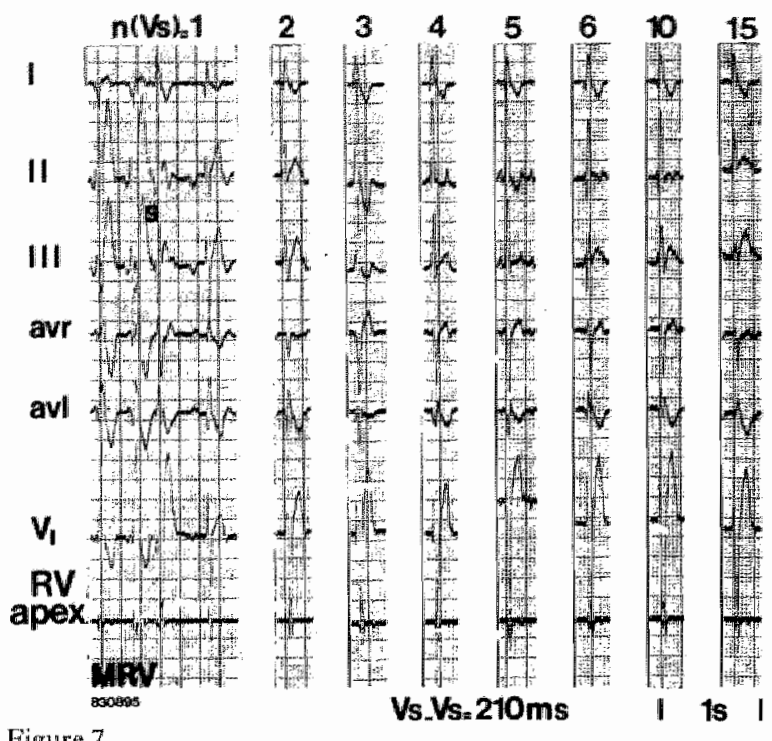

Figure 7

The influence of the number of stimuli on the first postpacing QRS-configuration during electrical stimulation distant to the dominant pacemaker sites.

Example form the same experiment as shown in figure 5 and 6 . The ventricular tachycardia originates in the left ventricular apex. The ventricles are stimulated from the midportion of the right ventricle (MRV). On increasing the number of stimuli the first postpacing QRS -complex changes from left ventricular apical to right ventricular basal and right ventricular anidportion.

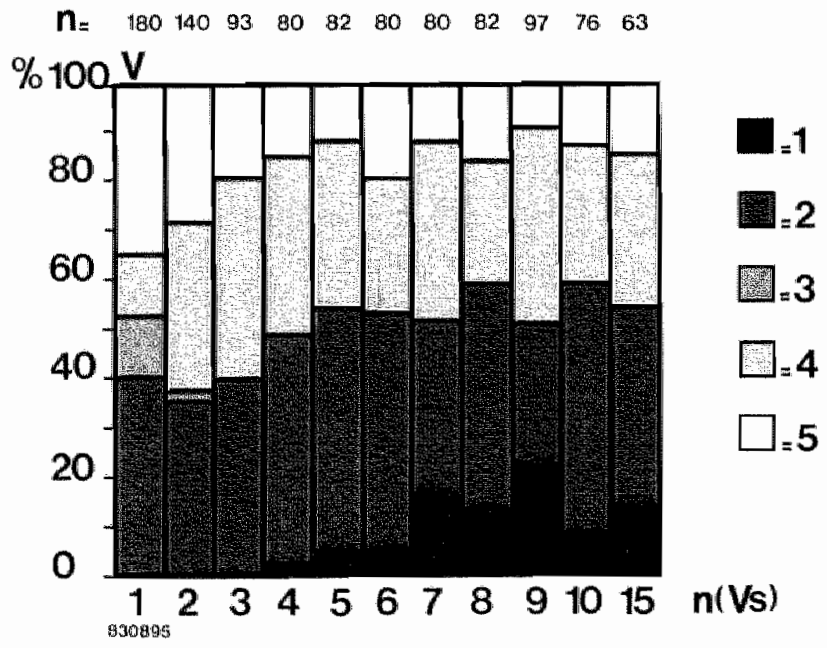

Figure \&

The influence of the number of stimuli on the first postpacing QRS-configuration during ellectrical stimulation distant to the dominant pacemaker site.

Diagram relating the configuration of all QRS-complexes (vertical axis) from the experiment of figure 7 to the number of stimuli (horizontal axis). The toral number of analyzed QRS-complexes is 1053 . The QRS-

configuration is indicated in the diagram (see figure 6). Note that on increasing the number of stimuli from 1 to 5 the QRS-configuration gradually changes from left ventricular apical to right ventricular basal. At higher number of stimuli more postpacing complexes originate at the site of stimulation. 
Figure 8 gives a diagram of all first postpacing QRS-complexes from this experiment. A similar behaviour of the first postpacing QRS-complex was found when only the last paced interval was altered.

Short extrastimulus intervals induced first postpacing QRS-complexes which originated close to the paced complexes and long extrastimulus intervals induced first postpacing QRS-complexes from the apex of the left ventricle.

Interestingly, further lengthening of the extrastimulus interval again induced a QRSconfiguration from close to the pacing site (figure 9). The length of the first postpacing interval during the last situation was about equal to the extrastimulus interval, whereas the shorter extrastimulus intervals resulted in first postpacing intervals being about 2 times as long as the extrastimulus interval (see diagram figure 9).

Figure 10 shows the result of the effect of an extrastimulus given with an interval duration long enough to lead to fusion with the spontaneous QRS-complex. This gives a QRSconfiguration which is identical to the last paced QRS-configuration. The implication of this finding will be discussed later.

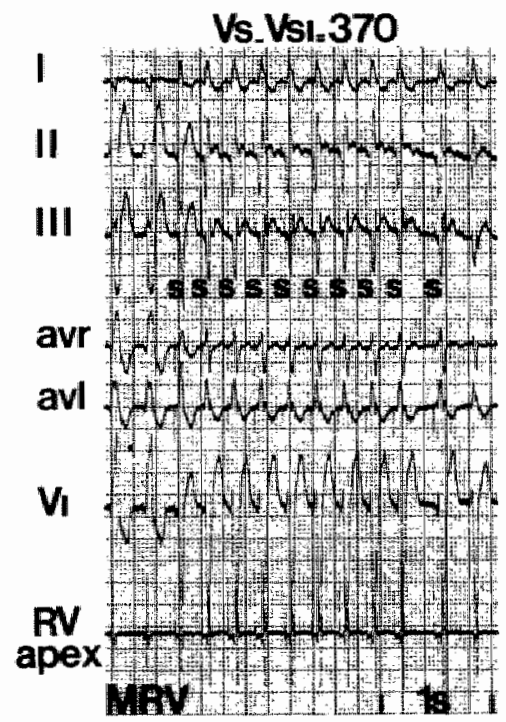

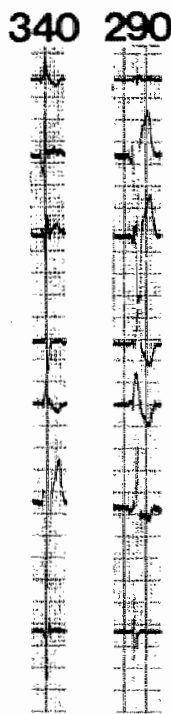

n(Vs).9

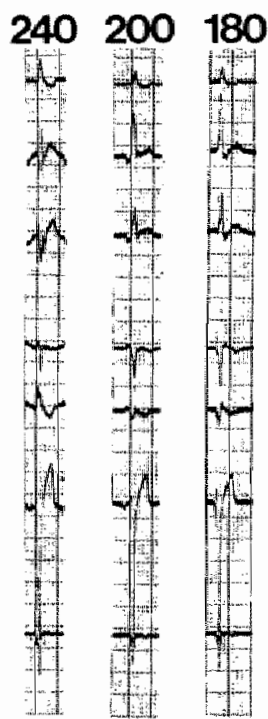

Vs.Vs.250ms $\mathrm{ms}$
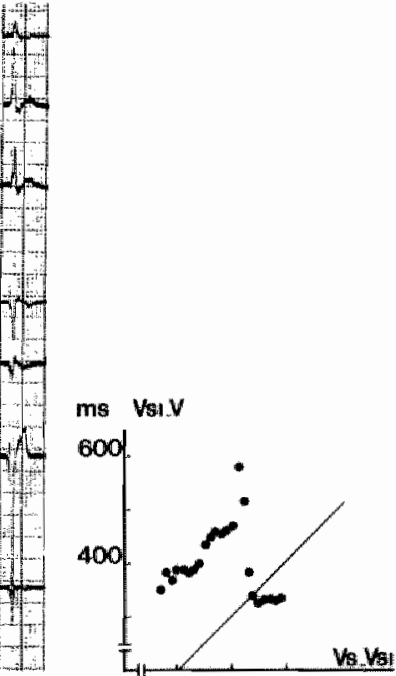

$200 \quad 400 \mathrm{~ms}$

Figure 9

The influence of extrastimuli on the first postpacing QRS-configuration.

Example from the same experiment as in figure 5-8. The spontaneous pentricular tachycardia originates in the left ventricular apex. Electrical stimulation is done at the midportion of the right ventricle. Basic pacing is donc with 9 stimuli and interstimulus intervals of $250 \mathrm{~ms}$. On decreasing the interstimulus interval the configuration of the first postpacing QRS-complex changes from left ventricular apical to a right ventricular basal and a mid right wentricular origin. However, note that the longest extrastimulus intervals induce aR Q-configuration suggesting an origin close to the stimullation site. The diagram shows the relation between the interstimulus interval (horizontal axis) and the length of the first postpacing interval. 
Although the configuration of the first postpacing QRS-complex frequently differed from the spontaneous tachycardia an identical configuration could be observed, especially, as show m above, after long pacing intervals. This finding suggests that the dominant tachycardia focus reacts similarly to electrical stimulation as the foci activated by pacing. During the tachycardia of figure 1.1 also the same QRS-configuration was observed after pacing as during the spontaneous tachycardia. Therefore the findings obtained during electrical stimulation were used for the interpretation of the mechanism of the spontaneously occurring tachycardia.

Figure 11 shows a group beating tachycardia, a finding which we have frequently observed before termination of sustained ventricular tachycardia and during episodes of nonsustained ventricular tachycardia. Characteristically, the groups are separated by long intervals ( $450 \mathrm{~ms}$ in this example) and show increasingly shorter intervals $(360,330$ and $290 \mathrm{~ms}$ respectively). The configuration of the QRS-complexes shows in this example increased narrowing of the QRS-width and a gradual change in axis. This group beating tachycardia lasted sufficiently long to be studied by single and paired stimuli and also by extrastimuli following basic pacing with 8 stimuli and different basic pacing intervals. Because the results of these different pacing modes were essentially the same, only the findings during pacing with one stimulus are presented (figure 12).

In the tracings shown in figure 12 pacing is done at the left ventricular apex. Long extrastimulus intervals (panel 1) resulted in narrow QRS-complexes (Ib, Ic,Id of the spontaneous ventricular tachycardia, see figure 11). Intermediate extrastimulus intervals are followed by the wide QRS-complex of the spontaneous ventricular tachycardia (Ia of figure 11) and short extrastimulus intervals induced a different QRS-complex (type II) originating closer to the stimulation site i.e. in the anterior fascicle of the left bundle branch.

In this experiment a sudden lengthening of the postpacing interval duration was always

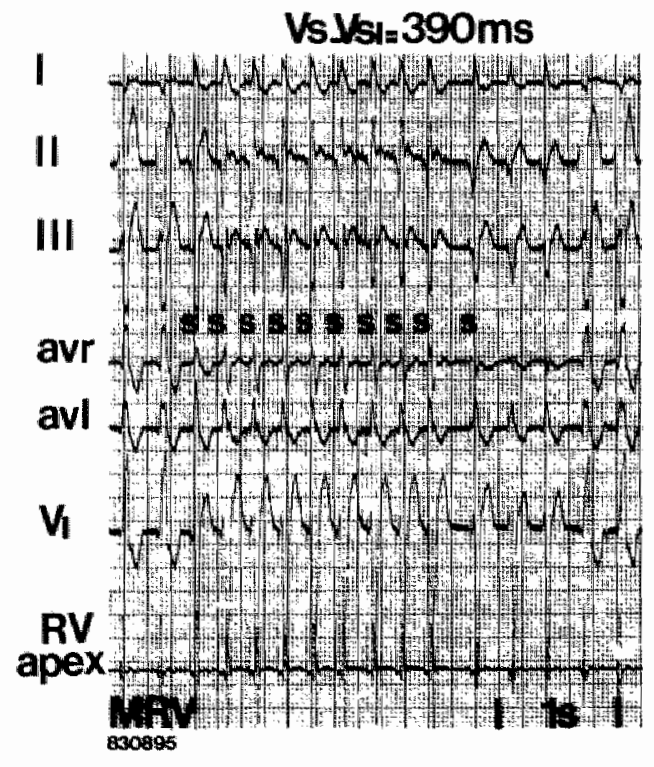

Figure 10

The effect of maximally long extrastimulus intervals. The extrastimulus is given after such a long interwal that the stimulated activation front fuses with spontaneous activation from the left ventricular apex. The first and subsequent QRS-complexes show an identical QRSconfiguration as the last stimulated QRS-complex. 
observed on shortening the extrastimulus interval inducing a narrow QRS-complex (panel 1) to the extrastimulus interval inducing a wide QRS-complex (panel 2). Further shortening of the extrastimulus interval resulted in a decrease of the interval (panel 3 and 4, diagram). The implications of these findings are discussed below.

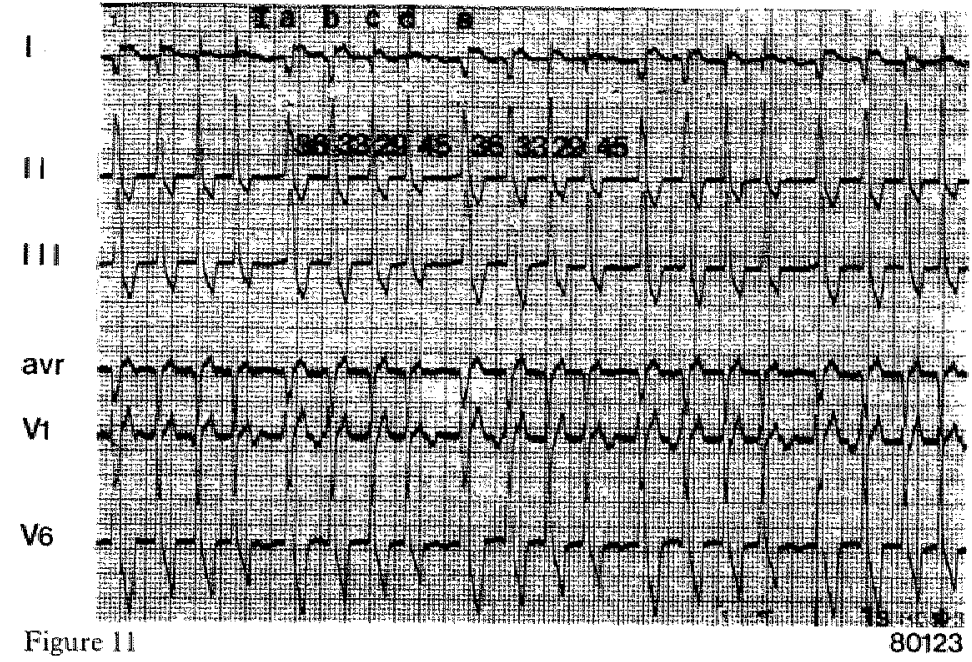

Group beating ventricular tachycardia.

Typical example of a tachycardia consisting of groups of 4 QRS-complexes. Each group shows shorter R-R intervals followed by a longer pause. Also increasing narrowing of the QRS-width is noted within cach group. The QRS-configuration suggests an origin in the basal part of the ventricular Purkinje system. The different types of QRS-complexes are coded as $I \mathrm{a}, \mathrm{b}, \mathrm{c}$, and d respectivelly.
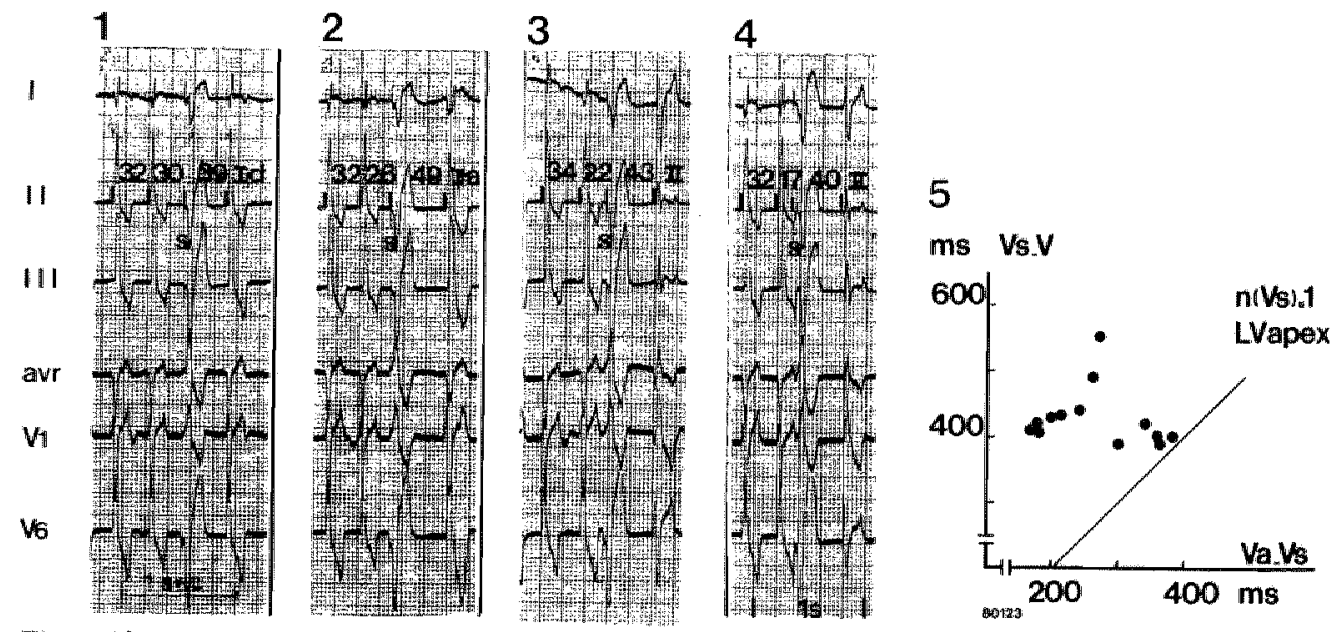

Figure 12

Effect of programmed electrical stimulation during the ventricular tachycardia shown in figure II.

During ventricular tachycardia a single stimulus is given with increasing prematurity at the left ventricular apex.

Decreasing the pacing interwal results in a sudden lengthening of the first postpacing interval (panel 1 panel 2) after which gradual shortening of the furst postpacing interval is seen (panel 2-4). The interval shift was always accompanied by the wider QRS-configuration (type Ia of figure 11). Panel 5 gives the relation between the premature beat interval $\left(V_{a}-V_{s}\right)$ and the post premante beat interval $\left(V_{s}-V\right)$. 


\section{Discussion}

\section{The spontaneous ventricular tachycardia}

The spontaneons ventricular tachycardia in our experiments most frequently had a QRS-configuration suggesting an origin in the apex of the left ventricle. These findings are similar to previous observations in the intact dog heart ${ }^{19,71}$. Electrode catheter recordings of the specific intraventricular conduction system in the intact dog heart have revealed that the left bundle branch system is a predilection site for digitalis-induced ventricular tachycardia ${ }^{19}$. The site of impulse formation was found to be located either proximal or distal in the left bundle branch system. Epicardial mapping techniques in open chest dogs have shown that during digitalis-induced ventricular tachcyardia earliest epicardial activation always occurred at or near the apex of the left ventricle ${ }^{71}$. The greater sensitivity of the left than the right ventricular conduction system for digitalis probably explains the prevalence of right bundle branch block configuration ${ }^{13,14,97}$ in digitalisinduced ventricular tachycardias occurring clinically ${ }^{118,140}$.

Also in vitro measurements of paired right and left canine Purkinje fibers showed a higher sensitivity for digitalis in the left system ${ }^{94}$. Several mechanisms have been proposed to explain this difference: differences in affinity of the glycoside to the $\mathrm{Na}^{+}-\mathrm{K}^{+}$ATP-ase, in the number of glycoside binding sites or qualitative differences in ventricular sarcolemmal binding sites ${ }^{9.4}$. The second most frequent ventricular tachycardia configuration found in our experiments is suggestive for an origin in the basal part of the right ventricle. Also in a small number of in vitro experiments right ventricular Purkinje fibers showed earlier signs of toxicity than left sided ones ${ }^{94}$.

Many authors have described that impulse formation in digitalis intoxication preferably originates within the specific conduction system ${ }^{19,52,71,93,119}$.

This finding is supported in our experiments by the observation that most of the first postpacing QRS-complexes had a fast initial upstroke, in contrast to the slow initial deflection of the paced QRS-complexes ${ }^{42}$.

\section{The effect of programmed electrical stimulation}

Programmed electrical stimulation close to one of these dominant pacemaker sites resulted in a first postpacing QR S-complex suggesting an origin from these sites (figure 2). Pacing distant to the dominant sites of impulse formation induced fusion of electrical activity from the predilection sites and from the stimulation site. This finding is in agreement with previous observations ${ }^{161}$ and has been explained as follows. Because the stimulation site is activated first by electrical stimulation a potential focus will discharge earlier than other ectopic pacemakers within the ventricles. The dominant sites of impulse formation will also become activated resulting in QRS-complexes based on fusion between both dominant sites of impulse formation and the stimulation site.

Interescingly the amount of fusion appeared to be dependent on the pacing rate and the duration of pacing. Changing one extrastimulus interval only resulted in a similar behaviour of the QRS-configuration as during regular pacing.

This behaviour of the QRS-configuration is in agreement with triggered activity as the underlying mechanism for impulse formation at the stimulation site. Such a mechanism is further supported by the behaviour of the first postpacing interval. As described 
previously ${ }^{43,161}$ this is similar to the behaviour of delayed afterdepolarizations during cardiac pacing. Foci distant to the origin of the spontaneous ventricular tachycardia and close to the stimulation site can become manifest because: 1) electrical stimulation with faster rates than the spontaneous tachycardia increases the amplitude of delayed afterdepolarizations enough to reach threshold; 2) during stimulation foci close to the stimulation site are the first which become activated. After pacing they can discharge prior to more distally located foci. Once the focus induces manifest ectopic activity, it will fuse with the ectopic activity from the dominant pacemaker. The amount of fusion depends on the time difference between the discharge of both foci. This time difference depends on 1) differences in escape interval of the focus at the dominant sites of impulse formation and the focus close to the stimulation site and 2) the conduction time between both foci.

When delayed afterdepolarizations induce the first postpacing QRS-complex, differences in escape interval can occur because of a) differences in the coupling interval and b) differences in steepness of the slope of the delayed afterdepolarization. In isolated Purkinje fibers studies ${ }^{94}$ both parameters have been found to differ in left and right Purkinje strands. As far as the conduction time between the pacing site and the site of spontaneous impulse formation is concerned, this is expected to vary depending on the pacing rate and the extrastimulus interval. Pacing with rapid rates may induce a slowing of conduction because of a pacing induced decrease of the diastolic membrane potential 98,121. Another mechanism for delay in conduction may be a pacing induced increase in the amplitude of delayed afterdepolarizations, which is also known to induce conduction block ${ }^{98,105}$. The relation between escape interval and conduction time to explain the QRS-configuration is illustrated in three different situations in figure 13. In the first situation the first postpacing QRS-configuration is similar to the spontaneous tachycardia configuration. Here the escape interval at the site of stimulation is longer than the escape interval of a dominant pacemaker site and two times the conduction time between the stimulation site and the site of spontaneous impulse formation (figure 13, panel 1). In the second situation the first postpacing QRS-configuration is similar to the QRSconfiguration during pacing. Now the escape interval at the site of stimulation is smaller than the escape interval at the site of spontaneous impulse formation and two times the conduction time between both sites (figure 13, panel 2).
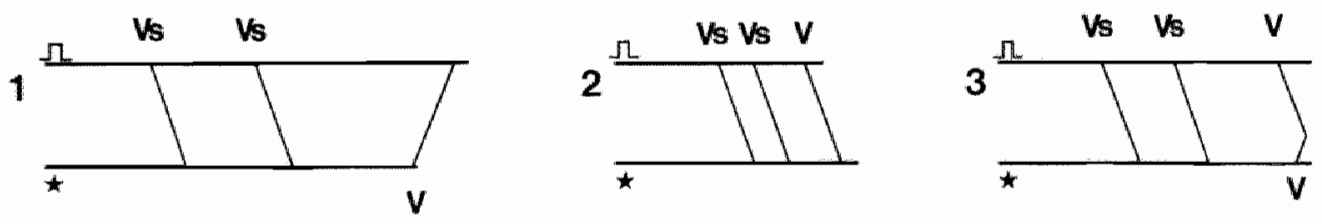

Figure 13

Diagrams illustrating the change in configuration of the first postpacing QRS-complex.

The unterstimulus interval is long in panel 1 , short in panel 2 and intermediate in pand 3 . The first postpacing interval is $150 \%$ of the paced cycle length. See text.

\footnotetext{
- - sire of stimulation

* site of impulse formation of spontaneous ventricular tachycardia

v site of origin of first postpacing QRS-complex.
} 
In the last panel the first postpacing QRS-configuration is a fusion complex between a configuration of the paced beats and the spontaneous tachycardia beats. The escape interval at the pacing site is longer than the escape interval at the spontaneous site of impulse formation and one time the conduction time between both foci but shorter than the escape interwal at the spontaneous site of impulse formation and two times the conduction time between both foci (figure 13, panel 3).

The finding that following long extrastimulus intervals also impulse formation was induced close to the stimulation site with an interval duration being equal to extrastimulus interval is of interest (figure 9). Previously it has been found that the interstimulus interval has a biphasic relation to the first postpacing interval ${ }^{43,87}$. Long pacing intervals induce first postpacing intervals which are equal to the pacing interval whereas short interstimulus intervals have a $1: 2$ relation to the first postpacing interval. This behaviour is explained by the fact that the amplitude of the first delayed afterdepolarization decreases at higher pacing rates whereas that of the second one increases $32,35,87,99,100,101$. The first postpacing QRS-complexes with an equal interval duration after long pacing intervals and originating close to the stimulation site (figure 9) are therefore likely to be induced by the first afterdepolarization. However one may argue that there is no relation to the preceding extrastimulus because its coupling interval is so long that it fails to enter the site of impulse formation. Against this explanation speaks the configuration of the first postpacing QRS-complex which suggests an origin close to the site of stimulation in contrast to those observed after shorter extrastimulus intervals. Although suggestive this does not completely exclude that the first postpacing QRS-complex was the result of the basic drive only. Evidence against the latter possibility is found in the observation shown in figure 10. The extrastimulus had such a long coupling interval that it fused with spontaneous electrical activity which, in view of the QRS-configuration, probably originated in the left ventricular apex. This extrastimulus was followed by a QRS-complex with an identical configuration as the stimulated QRS-complex. This observation suggests that the QRS-complexes occurring after an interval about equal to the extrastimulus interval and with a QRS-configuration of the stimulated QRS-complexes are indeed evoked by the extrastimulus. This indicates that they are the result of the first delayed afterdepolarization. The change in QRS-configuration shown in figure 9 can then be explained as follows:

From short to long extrastimulus intervals the second delayed afterdepolarization at the stimulation site becomes gradually smaller and exerts increasingly less influence on the QRS-configuration. Therefore increasingly less influence of electrical activity from the stimulation site is observed in the first postpacing QRS-complex. The first delayed afterdepolarization however increases and when it becomes sufficiently high it induces again ectopic activity from the site of stimulation.

So we consider this "biphasic behaviour" of the QRS-configuration following extrastimuli as another argument for the triggered nature of the first postpacing QRS-complex.

\section{Possible mechanism of the spontaneous tachycardia}

The observation of first postpacing QRS-complexes with an identical QRS-configuration as the spontaneous ventricular tachycardia (figures 11 and 12) suggests that the dominant 
pacemaker focus behaves similarly to electrical stimulation as sites of impulse formation induced by pacing.

The findings during programmed electrical stimulation of such a focus can be extrapolated to the spontaneous ventricular tachycardia (see figures 14-15). The pacing study revealed that the wide QRS-complex was probably induced by the second delayed

afterdepolarization. Also during the spontaneously occurring ventricular tachycardia the wide QRS-complex always happened after a longer pause. Interpreting this pause as the result of a subthreshold first delayed afterdepolarization the mechanism of the tachycardia may be as shown in the diagram of figure 14 . The second delayed afterdepolarization

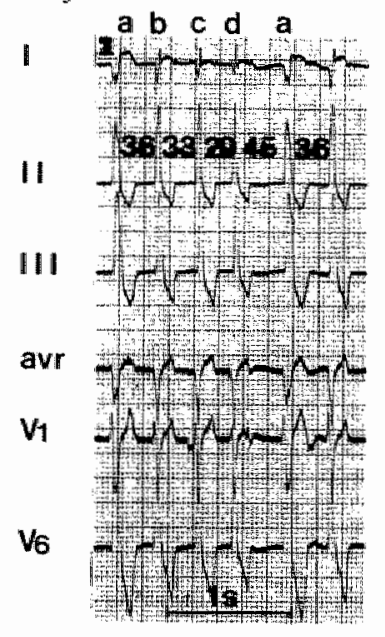

Figume 14 Interpretation of digitalis-induced group beating ventricullar tachycardia. See text.

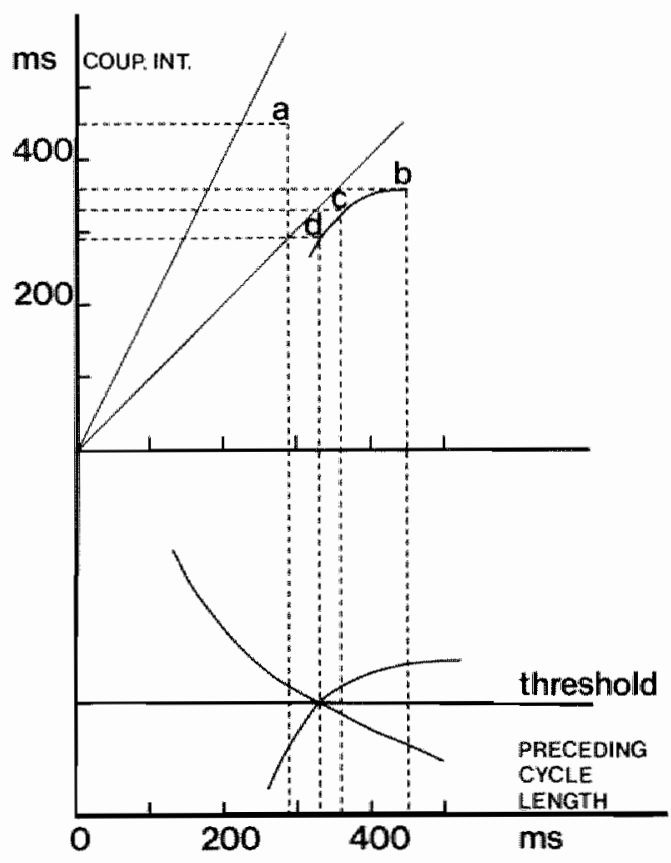

Figure 15

Diagram of the possible underlying mechanism of the wentricular tachycardia of figure if relating the length of given tachycardia interval to its preceding interval. See text. 
induces the wide QRS-complex. This long pause results in the induction of a suprathreshold first delayed afterdepolarization with a shorter interval duration than the preceding cycle. Because of this shortening the next impulses will be increasingly more accelerated until the preceding interval duration becomes so short that the first afterdepolarization becomes subthreshold, after which the second becomes manifest. After this longer interval a first delayed afterdepolarization will be induced with a long interval, and the sequence starts again. This is diagrammatically presented in figure 15 . In this diagram the amplitude (lower panel) and coupling interval (upper panel) of the first and second delayed afterdepolarizations is plotted as a function of the preceding cycle length. A given coupling interval of the spontaneous tachycardia is plotted as a function of its preceding interval. It is shown how the coupling interval of the QRS-complex Ib,c and $\mathrm{d}$ of figure 11 , are thought to be induced by the first delayed afterdepolarization. These first delayed afterdepolarizations have increasingly shorter intervals until subthreshold values are attained, after which the second delayed afterdepolarization can trigger. We consider this mechanism the most likely explanation for the frequently observed group beating ventricular tachycardia in digitalis intoxicated dogs. Comparing the intervals of the hypothesized first delayed afterdepolarizations in the presented tachycardia with the coupling intervals in isolated Purkinje fiber studies, it is noticed that they are shorter than the coupling intervals found in isolated Purkinje fiber studies ${ }^{32,87}$. In the latter experiments no first delayed afterdepolarizations were observed after pacing intervals shorter than $350 \mathrm{~ms}$. We think however that this does not exclude our interpretation of the spontaneous ventricular tachycardia. One difference with our experiment is the absence of sympathetic tone in isolated Purkinje fibers. Sympathetic stimulation is known to increase the amplitude of delayed afterdepolarizations ${ }^{50}$.

In conclusion our results indicate that digitalis-induced ectopic impulse formation occurs in predilection sites in the ventricle. Ectopic impulse formation elsewhere can however be induced by electrical stimulation resulting in fusion between activation from the dominant pacemaker site and the stimulation site. The dependency of the amount of fusion on the rate and duration of pacing and the duration of the extrastimulus interval results in large number of fusion complexes. Based on the behaviour of the QRS-configuration to pacing the most likely mechanism for the first postpacing QRS-complex is triggered activity. Extrapolating the findings of programmed electrical stimulation to the spontaneous ventricular tachycardia makes triggered activity the most لikely explanation for group beating ventricular tachycardia.

\section{Clinical implications}

Ectopic impulse formation during digitalis intoxication can be induced not only in predilection sites but also in other parts of the ventricle when they get the opportunity to discharge earlier than the dominant focus. We could demonstrate this by pacing distant from the dominant focus. Theoretically this can be used as a test in patients suspected of digitalis intoxication. The ability of different foci to discharge may explain the occurrence of ventricular ectopic beats with the same coupling interval but different QRSconfiguration during digitalis intoxication ${ }^{38,118,140}$. It is likely that concomitant localized pathological conditions like ischemia, myocardial infarction, cardiomyopathy etc. will facilitate triggering and will make these areas prone to discharge ectopic impulses. Our 
observations make clear that in the patient with digitalis intoxication cardiac pacing and cardioversion can be dangerous. Especially pacing at higher rates will facilitate the induction of ectopic impulse formation based upon triggered activity. In the patient with digitalis intoxication the pacing rate should therefore be as low as hemodynamically acceptable. Anxiety, exercise or other stimuli increasing sympathetic stimulation should be avoided in patients with digitalis intoxication because they will increase the amplirude of delayed afterdepolarizations. Our observations should be a stimulus for the development of drugs specifically influencing the occurrence of delayed afterdepolarizations. In this regard calcium antagonists are of interest because they counteract digitalis induced intracellular calcium accumulation ${ }^{74}$ which is thought to be the mechanism of delayed afterdepolarization ${ }^{70}$. Also lidocaine like drugs which block the transient inward current ${ }^{29}$ inducing delayed afterdepolarization have to be considered 99 



\section{Effect of overdrive stimulation on the normal idioventricular rhythm.}

by

Anton PM Gorgels, MD, Bram de Wit, MD, Henrierte DM Beekman, Willem RM Dassen, PhD, Hein JJ Wellens, MD, Deparument of Cardiology Annadal Hospital MAASTRICHT The Netherlands

\section{Abstract}

The effect of overdrive stimulation was studied in $\mathrm{I} 2$ conscious dogs with formalin induced complete atrioventricular block. The pacing protocol included regular pacing at increasing rates (60 to $300 / \mathrm{min}$.), regular pacing using an increasing number of stimuli (between ro and 200) and basic pacing followed by extrastimuli. The last prepacing interval, and the first and second postpacing interval were measured. Overdrive suppression of the first and second postpacing interval was observed following interstimulus intervals between rooo and $400 \mathrm{~ms}$. Decreasing the interstimulus interval to $200 \mathrm{~ms}$ resulted in shortening of the first postpacing interval in all experiments.

QRS-complexes emerged which behaved differently to programmed stimulation as during normal overdrive suppression. These complexes were termed: premature escape beats. They were readily induced following short and fast overdrive pacing and characterized by a first postpacing interval which was I) not influenced by an increase in number of stimuli until suppression occurred at a critical number of stimuli, 2) followed by a longer second postpacing interval, 3) already inducible by single extrastimuli following basic pacing. Premature escape beats frequently originated at the same site as the spontaneous ventricular rhythm and the (overdrive suppressed) second postpacing QRS-complex. Entrance block could be excluded as the underlying mechanism. Triggered activity is suggested as the most likely mechanism for these premature escape beats.

In the preceding chapters we described that an increase in pacing rate during digitalis intoxication results in shortening of the first postpacing interval. These findings are in agreement with those of other investigators $7,159,160,161$ and possibly due to delayed afterdepolarizations $17,18,32,35,48,87,97$.

This behaviour of the first postpacing interval is in contrast with that of the normal idioventricular pacemaker which usually becomes increasingly suppressed following higher pacing rates ${ }^{72,75,76,77,127}$. Also in partially depolarized Purkinje fibers the induction of overdrive suppression is possible but related to the maximal diastolic potential and the duration of pacing ${ }^{22}$.

We considered the possibility that the observed concordant relation with the interstimulus interval during digitalis toxicity was, at least in part, related to the pacing mode. Therefore, experiments were performed without digitalis intoxication using similar pacing protocols. 


\section{Methods}

The experiments were performed in 12 mongrel dogs of either sex with a body weight between 25 and $30 \mathrm{~kg}$.

Through a right thoracotomy unipolar electrodes were fixed intramurally into the myocardium of the free wall of the right ventricle. Atrioventricular block was made by injecting formalin $37 \%$ in the region of the His bundle ${ }^{114}$. The experiments were performed without any premedication at least one week after the creation of complete AV-block. This one week period of rest was necessary to avoid episodes of spontaneous ventricular tachycardia, which are known to occur during the early postoperative period after the creation of artificial AV-block ${ }^{59,128}$.

Six electrocardiographic leads $\left(I, I_{1}, I I I, A V R, A V L\right.$ and $\left.V_{1}\right)$ and one epicardial lead were simultaneously registered (using a paper speed of $100 \mathrm{~mm} / \mathrm{s}$ ) on an ink-jet recorder and stored on tape. Electrical stimulation was done with a programmable stimulator having a synchronizing circuit. Unipolar stimuli were given with a stimulus strength of wice the diastolic threshold. Between consecutive stimulation trains enough time was allowed (2-5 minutes) for the ventricular rhythm to regain its prepacing rate.

\section{The stimulation protocol}

Overdrive stimulation was started by giving 10 stimuli at an interstimulus interval of 1000 $\mathrm{ms}$. Then in each stimulation train the interstimulus interval was shortened from 1000 to $200 \mathrm{~ms}$ in steps of 50-200 ms. The procedure was repeated with a higher number of stimuli. $10,20,50,100$ and 200 stimuli were given.

Also extrastimuli were given following regular pacing with 9 stimuli. Basic pacing was followed by an extrastimulus at $1000 \mathrm{~ms}$. The extrastimulus interval was shortened in steps of $50 \mathrm{~ms}$ up to $200 \mathrm{~ms}$. This was done for basic pacing intervals of 900,500 and 200 ms.

In 4 experiments extrastimuli were also given following regular pacing using 50 stimuli and a basic pacing interval of $400 \mathrm{~ms}$.

The last interval before stimulation and the first and second interval following stimulation were measured.

\section{Results}

The interval duration of the spontaneous ventricular rhythm was estimated by measuring the last interval before each stimulation train. The mean of 240 intervals in each of the 12 dogs was $1305 \pm 210 \mathrm{~ms}$ (ranging from 1030 to $1680 \mathrm{~ms}$ ).

\section{The effect of overdrive stimulation with 10 stimuli}

Because 10 stimuli were frequently used during the experiments with digitalis intoxication we used similar trains during the control experiments. It was found that decreasing the interstimulus interval resulted in a variable amount of overdrive suppression. However, irrespective of the amount of overdrive suppression, at a critical interstimulus interval always a pacing rate dependent decrease of the first postpacing interval duration occurred (figure 1). 


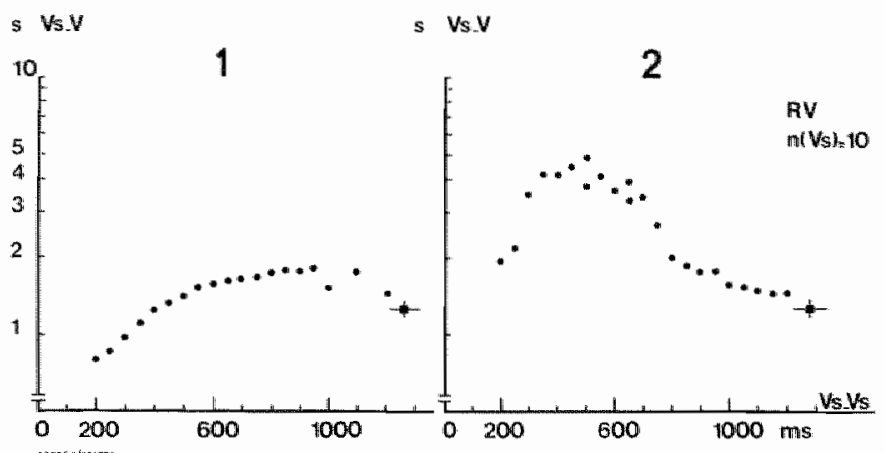

Figure 1

The effect of changing the interstimulus interval.

Two examples of overdrive stimulation with 10 stimuli from different dogs. Electrical stimulation is done from the right ventricle (RV). The first postpacing interval is plotted as function of the interstimulus interval. The vertical axis has a logarithmic scale. The black squares indicate the mean and standard deviation of the last prepacing interval.

The left panel shows the dog with the shortest first postpacing intervals and right panel rhe dog with the longes: escape intervals of a series of twelve dogs. Botlo panels show a pacing rate dependen decrease of the first postpacing interval starting at a critical interstimulus interval.

In most experiments this happened following interstimulus intervals of $600-400 \mathrm{~ms}$. The decrease in interval duration could be so marked that values below the interval duration of the spontaneous ventricular rhythm were attained (figure 1, panel 1).

Because this unexpected concordant relation between the interstimulus interval and the first postpacing interval was similar to that observed during digitalis toxicity and also because it was a consistent finding in twelve consecutive dogs, it seemed of interest to study this phenomenon in more detail. We did so by programmed electrical stimulation (this chapter) and by drugs specifically influencing mechanisms of impulse formation (chapter 7).

\section{The effect of higher numbers of stimuli on the first postpacing interval}

The same pacing procedure using a higher number of basic stimuli resulted in an increase of overdrive suppression of the first postpacing interval. This was observed when interstimulus intervals between 1000 to $400 \mathrm{~ms}$ were used. The duration of the first postpacing interval was dependent on the interstimulus interval and number of stimuli. The first postpacing interval was the longest one, followed by gradual recovery of the rhythm within 2 to 5 minutes. The longest first postpacing interval was usually observed after pacing with interstimulus intervals of $400 \mathrm{~ms}$ (figure 2, panel 1). Decreasing the interstimulus interval from 400 to $200 \mathrm{~ms}$ resulted in shorter postpacing intervals. The first postpacing interval showed two types of responses (figure 2). In the first type the first postpacing interval was shorter but subsequent intervals showed normal gradual recovery from overdrive pacing (figure 2, panel 2). So, these responses were characterized by less but otherwise normal overdrive suppression. In the second type of responses the first postpacing interval was even shorter but was followed by a longer second postpacing interval and thereafter by normal recovery from overdriwe pacing (figure 1, panel 3). As will be shown below the response to programmed electrical stimulation of the latter 

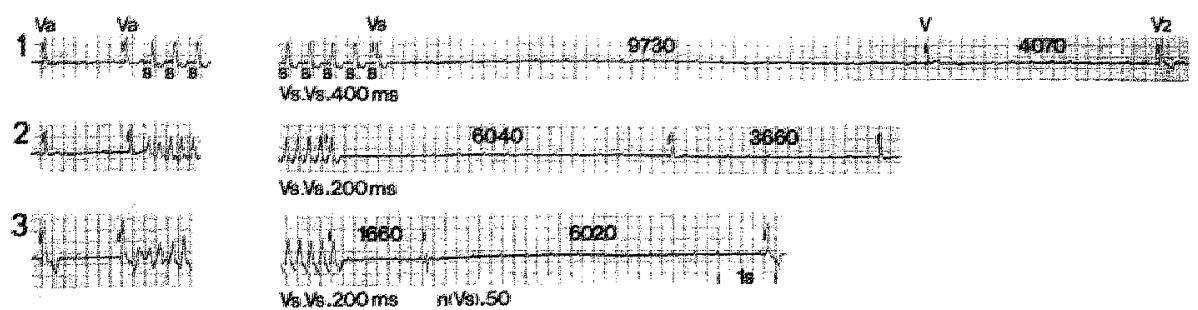

Figure 2

Owerdrive suppression and premature escape beats.

Tracings from two different dogs showing the decrease in duration of the first postpacing interval on shortening the interstimulus interval from 400 (first panel) $600 \mathrm{~ms}$ (second and third panel). In all panels 50 stimuli are used. Following interstimulus intervals of $400 \mathrm{~ms}$ (firs panel) marked overdrive suppression occurs and the first postpacing interval is lowger than the second one. In the second tracing shortening of the first (and second) postpacing interval is observed in comparison to panel 1 . In the third panel the first postpacing interval is much shorter than in panel $\mathbb{1}$ (premature escape besat) with the second postpacing interval now being longer.

group of first postpacing QRS-complexes differed from the behaviour of normal overdrive suppression. Because their interval duration is shorter than expected they will be termed "premature escape beats".

In the examples of figure 2 the differences in interval duration of the suppressed responses and premature escape beats were marked which made the differentiation of both types of responses easy. However when overdrive suppression was less marked, for instance after less stimuli or under increased sympathicotonia, the intervals following pacing were shorter and differentiation between the suppressed responses and premature escape beats by the interval duration alone could be difficult. Therefore we investigated whether the relation between the first and second postpacing interval as shown in panel 2 and 3 of figure 2 was a useful discriminator between both types of responses irrespective of the absolute duration of the first postpacing interval.

For this reason the second postpacing interval for each stimulation train was plotted as a function of the first postpacing interval. In figure 3 the results are shown for pacing trains of 50 stimuli and interstimulus intervals of $200 \mathrm{~ms}$. Data from 289 pacing trains are presented, derived from 32 experiments in 6 dogs. As shown the first and the second postpacing interval could have any value between 1000 and $8000 \mathrm{~ms}$. However the first postpacing interval was not at random shorter or longer than the second one. First postpacing intervals below $\pm 2000 \mathrm{~ms}$ had a shorter interval duration than their second postpacing interval in almost all cases (premature escape beats). Longer first postpacing intervals (more than $\pm 2000 \mathrm{~ms}$ ) usually had a shorter second postpacing interval (the less suppressed responses).

So, based on the relation between the first and the second postpacing interval, two different populations of responses after overdrive stimulation can be recognized. Therefore we used this relation to differentiate between suppressed beats and premature escape beats. Figure 4 and table 1 show the combined results from 6 experiments in different dogs following pacing with 10 and with 50 stimuli. The mean of the first and the second postpacing interval is plotted on a logarithmic scale as a function of the interstimulus interval $(1000,800,400$ and $200 \mathrm{~ms})$. Every stimulation program was repeated 10 times during each experiment. 


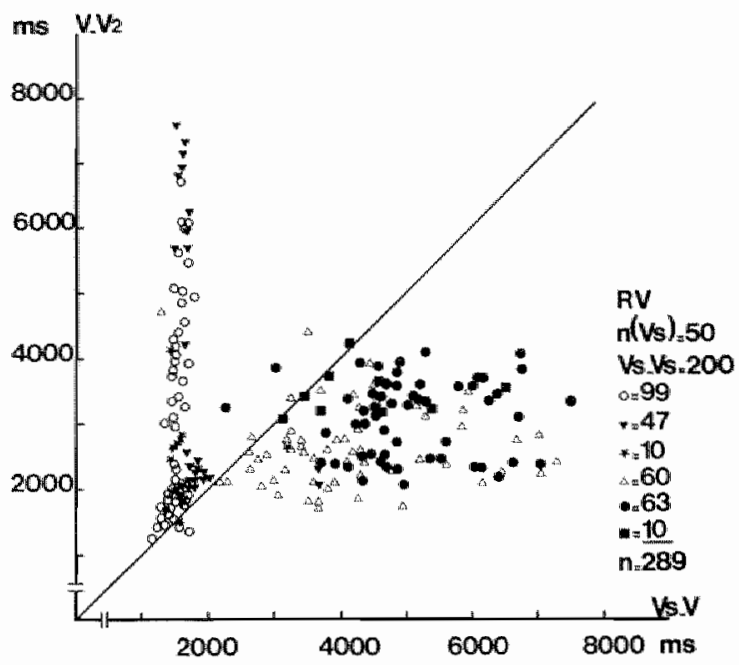

Figure 3

The relation between the first and the second postpacing interval.

Combined results from 6 different dogs following stimulation trains of 50 stimuli and $200 \mathrm{nns}$.

Horizontal axis: first postpacing interval.

Vertical axis: second postpacing interwal.

Symbols indicate different animals. The figure after the symbol indicates the number of stimulation trains in each dog. When the stimulation train led to identical first and second postpacing intervals, a single symbol is used to present the result of multiple stimulation trains.
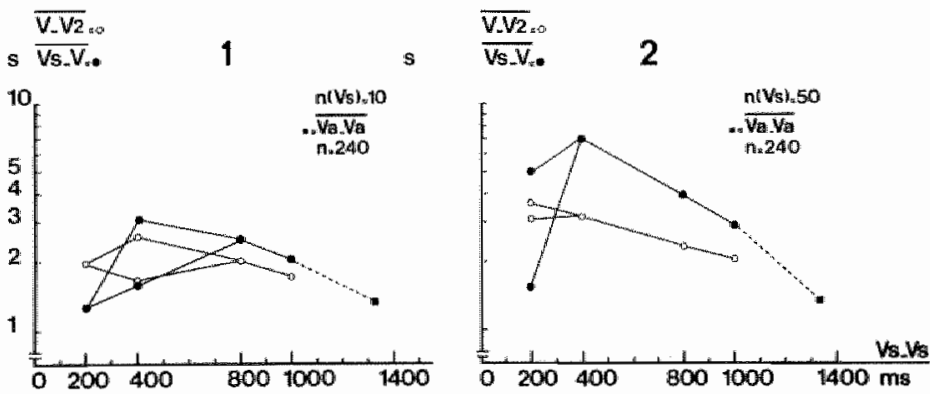

Fỉgure 4

Overdrive suppression and premature escape beats.

Combined results from 6 dogs. Pacing is done 10 times in each experiment in each dog using 10 and 50 stimuli.

Panel 1: pacing with 10 stimuli.

Panel 2: pacing with 50 stimuli.

Horizontal axis: interstimulus interval.

Vertical axis (logarithmic scale $y:$ first $\left(V_{5}-V\right)$ and second postpacing interval $\left(V-V_{2}\right)$.

Squares : mean of the last prepacing interval.

Black dors : mean of the first postpacing intervals.

Opendots : mean of the second post pacing intervals.

See text. 
Following pacing with 50 stimuli (panel 2) a pacing rate dependent increase of overdrive suppression occurs between interstimulus intervals of 1000 and $400 \mathrm{~ms}$. In almost all cases the first postpacing interval was longer than the second one (see table 1). Further shortening of the interstimulus interval to $200 \mathrm{~ms}$ resulted in overdrive suppression in three dogs and in premature escape beats in the other three. Following pacing with 10 stimuli again a pacing rate dependent increase in interval duration was observed between interstimulus intervals of 1000 and $400 \mathrm{~ms}$ (figure 4, panel 1). Following interstimulus intervals of $400 \mathrm{~ms}$ also premature escape beats were found after 17 stimulation trains in 3 dogs (see table 1). Following interstimulus intervals of $200 \mathrm{~ms}$ almost all first postpacing intervals in all dogs were premature escape beats.

\section{Further characteristics of premature escape beats}

As has been mentioned before the less overdrive suppressed QRS-complexes following $200 \mathrm{~ms}$ intervals behaved similarly as normal overdrive suppression. Their interval duration increased on increasing the duration of pacing (figure 5). Premature escape beats however showed different characteristics. 1) They were less frequently induced at a higher number of stimuli (table 1). 2) On increasing the number of stimuli their interval duration remained largely unchanged whereas the second postpacing interval gradually prolonged. 3) Their interval duration had a concordant relation to the interstimulus interval. 4) Also, premature escape beats could be induced by extrastimuli following regular pacing.

Table 1

Overdrive suppression and premature escape beats...

\begin{tabular}{|c|c|c|c|c|c|c|}
\hline \multicolumn{7}{|c|}{$V_{n}-V_{n}:: 1314 \pm 162(n=480)$} \\
\hline$n\left(V_{\mathrm{s}}\right)$ & $V_{s}-V_{s}$ & $V_{s}-V$ & SD & $\mathrm{V}-\mathrm{V}_{2}$ & SD & $\mathrm{n}$ \\
\hline \multirow[t]{7}{*}{50} & 1000 & 2030 & 0 & 2050 & 0 & $\mathbb{1}$ \\
\hline & & 2893 & 18.65 & 2033 & 765 & 59 \\
\hline & 800 & 1755 & 0 & 1810 & 0 & 1 \\
\hline & & 3925 & 2673 & 2291 & 681 & 59 \\
\hline & 400 & 7359 & 2398 & 3143 & 630 & 60 \\
\hline & 200 & 1507 & 188 & 3625 & 2298 & 29 \\
\hline & & 5027 & 1157 & 3096 & 675 & 31 \\
\hline \multirow[t]{6}{*}{10} & 1000 & 2073 & 1020 & 1719 & 593 & 60 \\
\hline & 800 & 2540 & 1400 & 2020 & 826 & 60 \\
\hline & 400 & 1540 & 180 & 1606 & 197 & 17 \\
\hline & & 3116 & 1308 & 2578 & 1062 & 43 \\
\hline & 200 & $\$ 505$ & 279 & 1967 & 726 & 59 \\
\hline & & 3130 & 0 & 3030 & 0 & 1 \\
\hline
\end{tabular}

Combined data from 6 experiments. All measurements are in milliseconds. For abbreviations see text.

SD: standard deviation.

n: number of stimulation trains. 

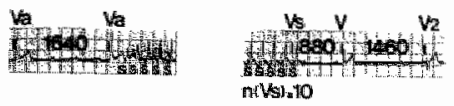

2

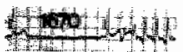

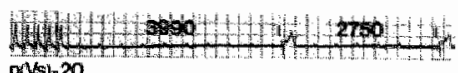

3
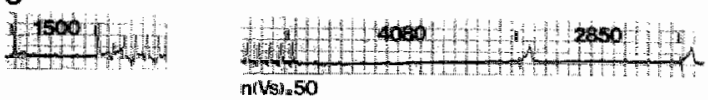

4

and 400

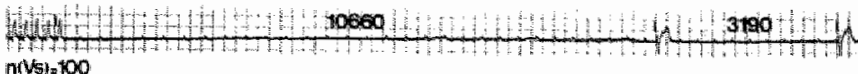

5

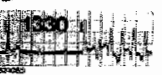

in(W5is 100

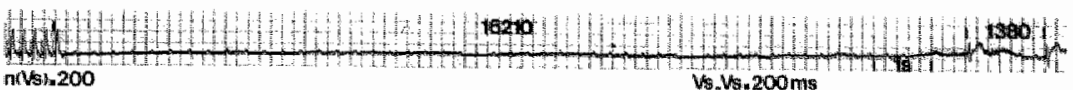

Figure 5

Overdrive suppression following pacing with $200 \mathrm{~ms}$ intervals. The effect of inereasing the number of stimuli.

In all panels the last prepacing interval, part of the stimulation procedure and wo postpacing intervalis atre shown.

The number of stimuli increases from 10 to 200 from the first to the fifth panel. The pacing interval is $200 \mathrm{~ms}$ in each panel.

In panel 1 a premacure escape beat is shown. From panel 2 all first postpacing QRS-complexes are overdrive suppressed but the interval duration is less than following pacing with $400 \mathrm{~ms}$ intervals. Note that the first postpacing interval gradually prolongs on increasing the number of stimuli and that starting with pane 2 the second interval is always shorter than the first one.
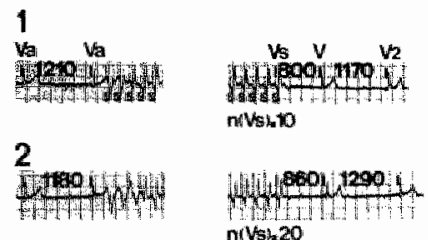

3
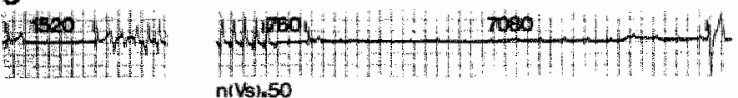

4
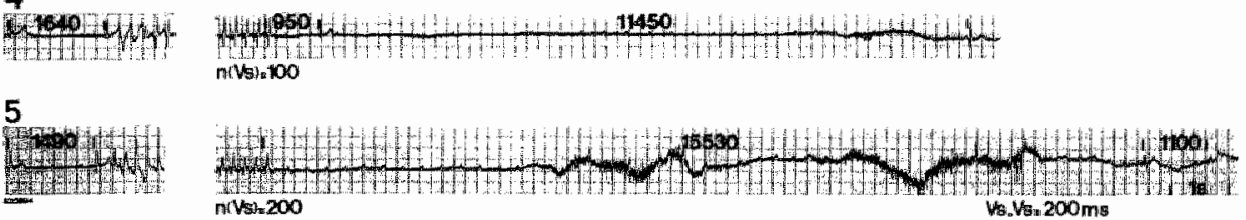

Figure 6

Premature escape beats. The effect of increasing the number of stimuli.

Arrangement of panels similar to figure 5 .

The first postpacing interval remains short, whereas the second one gradually prolongs. At 200 stimuli the first postpacing interval becomes wery long, the second interval now being shorter than the first one. 


\section{Suppression of premature escape beats by increasing the number of stimuli}

Increasing the number of stimuli resulted in the suppression of premature escape beats. This is illustrated in the experiment shown in figure 6 . In this figure in each panel the last prepacing interval, part of the stimulation procedure and two postpacing intervals are shown. The number of stimuli increases from 10 to 200 from the first to the fifth panel. The interstimulus interval is $200 \mathrm{~ms}$ in each panel.

It is shown that premature escape beats are induced following $10,20,50$ and 100 stimuli. Following 200 stimuli, however, no premature escape beat is observed: the first postpacing interval is markedly prolonged and the second postpacing interval is shorter than the first one. Following pacing with 10 stimuli and interstimulus intervals of $200 \mathrm{~ms}$ premature escape beats were observed in all dogs; following pacing with 50 stimuli in 6 out of 12 dogs and following 200 stimuli in 1 out of 12 dogs. In 6 of these dogs the reproducibility of these findings was tested (see figure 4, table 1 and 2 ). Following pacing with 10 stimuli and $200 \mathrm{~ms}$ interstimulus intervals premature escape beats were observed in 183 out of 187 stimulation trains in 6 dogs. Following 50 stimuli in 3 dogs premature escape beats occurred in all 175 stimulation trains. In the other three dogs no premature escape beats were induced after all stimulation trains.

On increasing the number of stimuli, suppression of premature escape beats occurred first at interstimulus intervals, which were usually followed by the greatest amount of overdrive suppression (600-400 ms). To illustrate this, the results of one experiment are presented in detail (figure 7). In table 2 the results are presented from 1757 stimulation trains of 35 experiments in the six other dogs studied with 10 and 50 stimuli and interstimulus intervals of $1000,800,400$ and $200 \mathrm{~ms}$. It is shown that, following 10 stimuli, more premature escape beats were induced the shorter the interslimulus interval. Following 50 stimuli the number of premature escape beats decreased, especially following interstimulus intervals of $400 \mathrm{~ms}$.

Table 2

The oceurresce of premature escape bests in relation to the number of stimuli and the interstimulus interval.

\begin{tabular}{lcc}
$n\left(V_{s}\right)$ & 10 & 50 \\
$V_{s}-V_{s}$ & & \\
1000 & $6 / 172$ & $20 / 206$ \\
800 & $19 / 182$ & $14 / 212$ \\
400 & $67 / 189$ & $3 / 304$ \\
200 & $183 / 187$ & $175 / 305$ \\
\hline
\end{tabular}




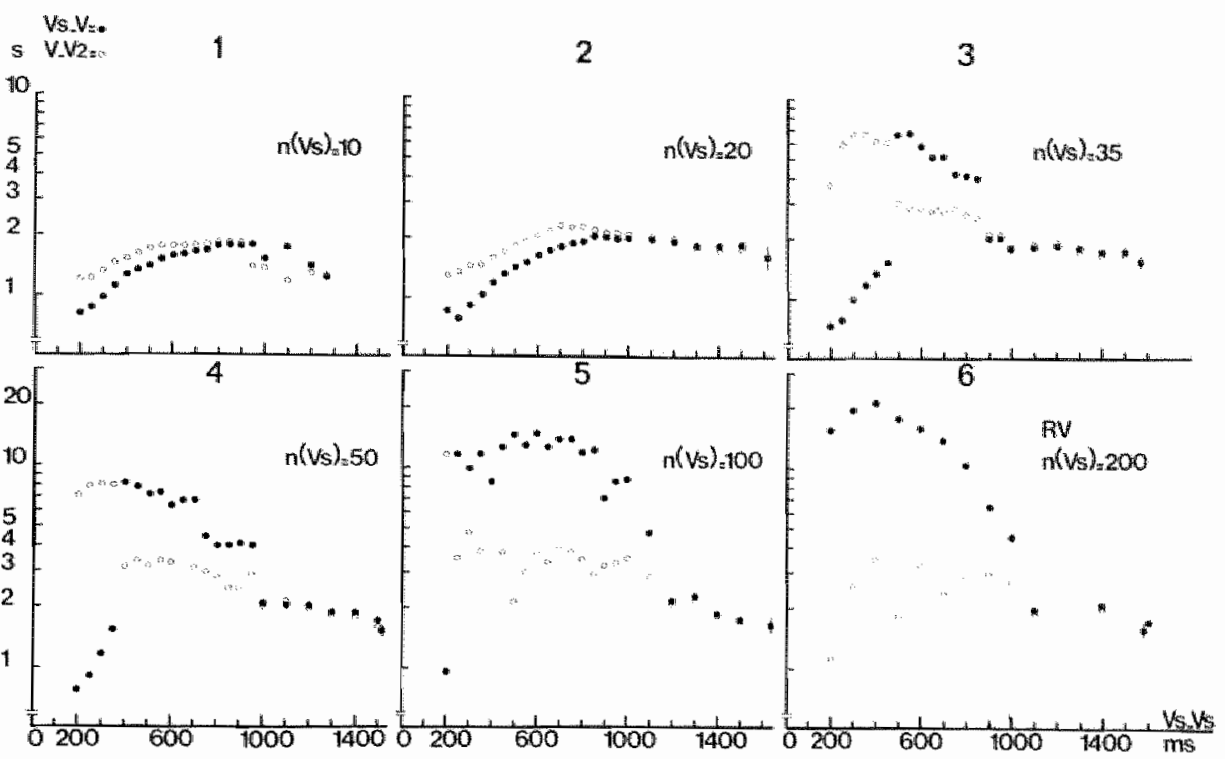

Figure 7

The effect of increasing the number of stimuli on overdrive suppression and premature escape beats. Alf results are derived from one experiment. In all diagrams the in terstinulus interval is plotted on the horizontal axis and the first and second postpacing interval on the vertical axis (logarimmic scale). Black squares indicate the mean of the last prepacing interval, black dots the first postpacing interval and open dots the second. postpacing interval. The number of stimuli increases from 10 to 200 from the first to the sixth panel, as ind icated in each panel $(\mathrm{n}(\mathrm{V} / \mathrm{s})$.

Note that 1) premature escape beats are induced over a wide range of interstimulus intervals following 10 and 20 stimuli. 2) Overdrive suppression occurs first at intemediate interstimulus intervals. 3) On increasing the number of stimuli the number of premature escape beats decreases. 4) At 200 stimuli all premature escape beats are suppressed.

\section{The effect of increasing the number of stimuli on the interval duration of premature escape beats}

As shown in figure 4,6 and 7 the interval duration of premature escape beats is largely unaffected by increasing the number of stimuli (until they are no longer induced on reaching a critical number of stimuli). This is further illustrated in figure 8 and table 3 . It is shown that the mean interval duration of premature escape beats does not increase at higher numbers of stimuli whereas the second postpacing interval substantially prolongs.

\section{The relation between the interstimulus interval and the interval duration of premature escape beats}

As shown in figure 7 the interval duration of premature escape beats decreased on decreasing the interstimulus interval. This was evaluated in three other dogs showing premature escape beats after pacing with 10 stimuli and interstimulus intervals of 400 and $200 \mathrm{~ms}$. The interval duration decreased from $1579 \pm 241 \mathrm{~ms}(\mathrm{n}=17)$ to $1259 \pm 89 \mathrm{~ms}$ $(\mathrm{n}=30, \mathrm{p}<0.001)$. 


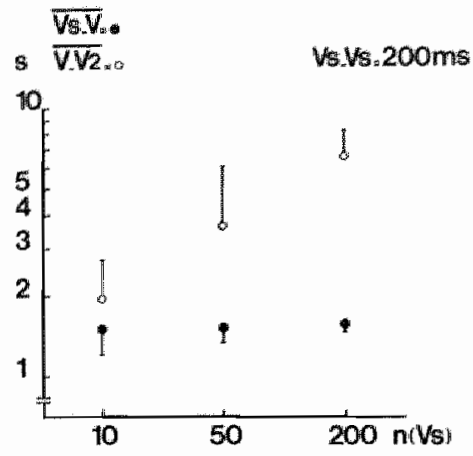

Figure 8

The influence of the number of stimuli on the interval duration of premature escape beats.

The norizon lat axis depicts the number of stimula and the vertical axis the first and second postpacing interval (on a logarithmic scale). The black cots represent the mean of the first postpacing interval and the open dots the mean of the second postpacing interval. The data are derived from 10 stimulation trains per experiment in 6 experiments in different dogs.

In all dogs premat ture escape beats were induced following 10 stimuli 59 of 60 stimulation trainsi.

Following 50 stimuli this was seen in 29 ont of 30 stimulation trains in 3 out of 6 dogs, and after 200 stimnlit in only 1 out of 6 dogs (in all 10 stimatation trains).

Note that the mean interwal duration of the premature escape beats does not change $(p>0.5)$ on increasing the number of stimuli, whereas the second postpacing interval makedly prolongs $(p<0.001)$.

(See also table 3).

Table 3

The effect of increasing the number of stimuli on premature escape beats.

\begin{tabular}{|c|c|c|c|c|}
\hline & $\begin{array}{l}\mathrm{n}\left(\mathrm{V}_{\mathrm{s}}\right)=10 \\
\operatorname{mean}\end{array}$ & SD & $\mathrm{n}$ & \\
\hline$V=V$ & 1505 & 279 & 59 & \\
\hline \multirow[t]{2}{*}{$\mathrm{V}-\mathrm{V}_{2}$} & 1967 & 726 & & \\
\hline & $\begin{array}{l}\mathrm{n}(\mathrm{V})=50 \\
\text { mean }\end{array}$ & SD & 助 & $\mathrm{p}(10 \mathrm{vs} 50)$ \\
\hline$V_{5}-V$ & 1507 & 188 & 29 & $>0.5$ \\
\hline \multirow{3}{*}{$\begin{array}{l}V_{-i} V_{2} \\
\mathbf{n}\left(\mathbf{V}_{4}\right)\end{array}$} & 3625 & 2298 & & $<0.001$ \\
\hline & & & & \\
\hline & mesan & $\mathrm{SD}$ & $\mathrm{n}$ & $p(50$ vs 200$)$ \\
\hline$V_{\mathrm{s}}-V$ & 1.539 & 41 & 10 & $>0.5$ \\
\hline$V-V_{2}$ & 6435 & 1712 & & $<0.001$ \\
\hline
\end{tabular}

All mestrements are in milliseconds.

For abbreviations see text.

$\mathrm{SD}=$ standard devintion.

$\mathrm{n}=$ number of stimulation trains. 


\section{The induction of premature escape beats by extrastimuli}

The influence of single extrastimuli was studied in 11 dogs. Following basic pacing with 9 the extrastimulus interval was shortened in steps of $50 \mathrm{~ms}$ from 1000 to $200 \mathrm{~ms}$. This was done during basic pacing intervals of 900,500 and $200 \mathrm{~ms}$. We found that in dogs, showing pronounced prematurity of the escape beats following 10 stimuli, the first postpacing interval also shortened by changing the extrastimulus interval only (figure 9). Decreasing the basic pacing interval resulted in additional shortening of the first postpacing interval.

We also observed that premature escape beats could be evoked by (single) extrastimuli following longer trains of basic pacing. This was even seen after basic pacing intervals of $400 \mathrm{~ms}$, which usually induced the greatest amount of overdrive suppression (see figure $10)$.

When this pacing protocol was performed in dogs showing overdrive suppression following $50 \times 200 \mathrm{~ms}$, these short first postpacing intervals could not be induced.

\section{Are premature escape beats due to entrance block of the pacemaker focus?}

Shortening of the escape intervals has also been observed in studying the behaviour of the sinus node during pacing increasing rates ${ }^{41,67,82}$.

Both entrance block and reentry have been postulated as explanation for the short postpacing escape interval of the sinus node. The described behaviour of the premature escape beats in our experiments makes entrance block as the underlying mechanism unlikely. Entrance block does not explain the increase of the second postpacing interval on increasing the number of stimuli, the suppression of premature escape beats by further increasing the number of stimuli, and the induction of premature escape beats by extrastimuli.

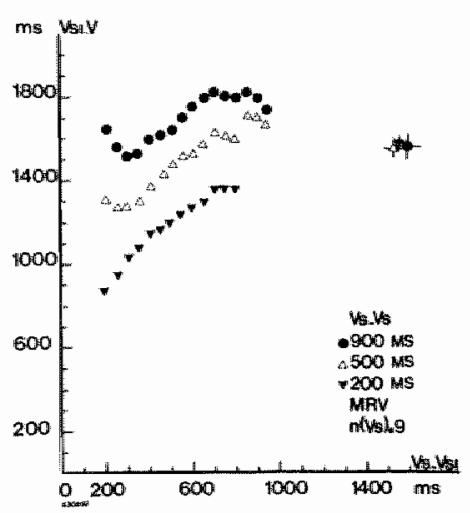

Figure 9

The effect of the extrastimulus interval on the first postpacing interval.

Basic pacing is done with 9 stimuli. Symbols indicate the basic pacing interval.

The mean and standard deviation of the last prepacing interval is shown on the right side of the figure.

See text. 


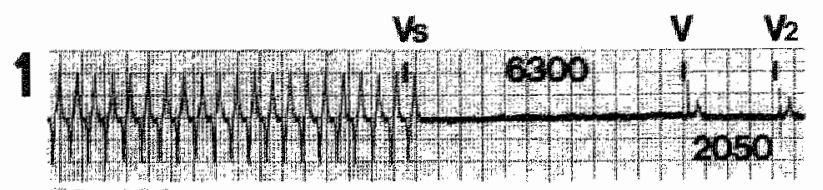

$50 \times 400 \mathrm{MS}$

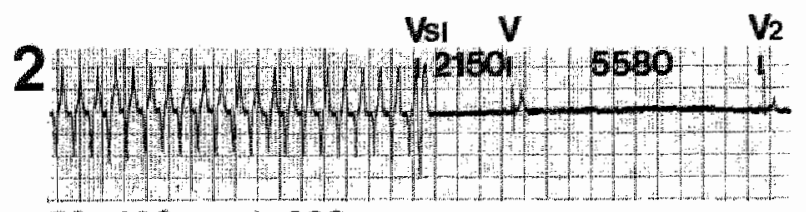

$50 \times 400 \mathrm{MS} 1 \times 200 \mathrm{MS}$

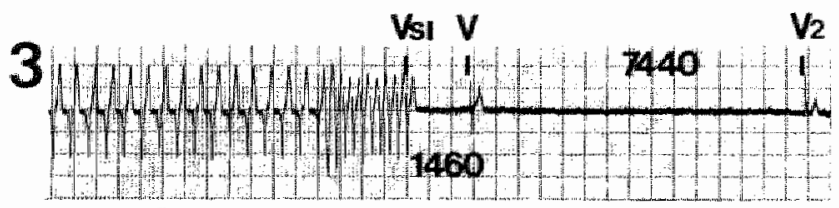

\section{$50 \times 400 \mathrm{MS} \quad 10 \times 200 \mathrm{MS}$}

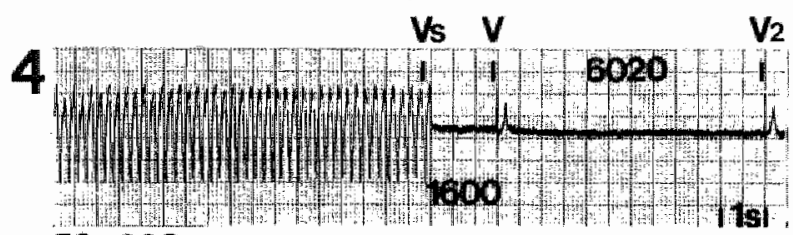

$50 \times 200 \mathrm{Ms}$
5

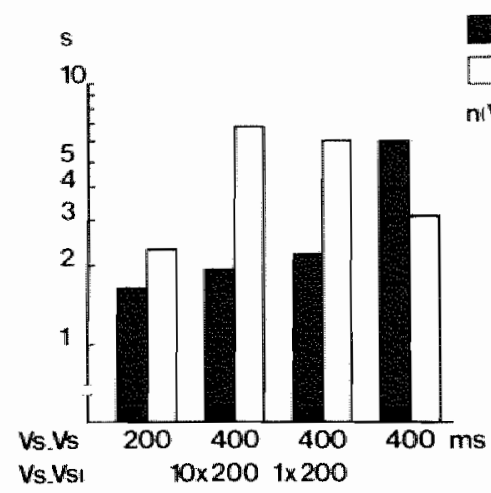

WS.V $\square \sqrt{v_{2}}$ nivs 50

Figure 10

The effect of extrastimuli following longer pacing trains.

Panels 1 to 4 show recordings of the effect of different pacing procedures on the first and second postpacing interval. Paper speed is $10 \mathrm{~mm} / \mathrm{s}$. Panel 5 shows the combined results in two dogs. The horizontal axis gives the different pacing modes. The vertical axis the first and second postpacing interval (logarithmic scale). Black bars: mean of the first pospracing interval.

Open bars: mesin of the second postpacing interval.

Pancl 1 shows the results of pacing with 50 stimuli a $400 \mathrm{mrs}$. Following stimulation overdrive suppression oceurs. Panel 2 shows regular pacing with 50 stimuli and $400 \mathrm{~ms}$, followed by a single extrastimulus after 200 ms. Now a short first posipacing interval and a longer second one is seen. In panel 3 the same basic stimulation procedure was followed by 10 extrastinuli with $200 \mathrm{~ms}$ interval.

Further shortening of the first post pacing interval is observed. Panel 4 shows the result of pacing with 50 st imuli having a $200 \mathrm{~ms}$ interval. A premature escape beat is followed by a longer second postpacing interval. Panel 5 shows these findings diagrammatically. The short first postpacing intervals following pacing wirh 200 ms are followed by longer second postpacing intervals. Long lirst postpacing intervals following pacing with $400 \mathrm{~ms}$ are followed by shorter second postpacing intervals. Addition of a single extrastimulus resulted in short first postpacing interwals and long second postpacing intervals (in 5 out of 8 stimulation trains). Addition of 10 stimuli of 200 ms induced further shorening of these premature escape beats (in 7 out of 8 stinulation trains).

Accepting that premature escape beats are based on a different mechanism than entrance block, the less overdrive suppressed beats following pacing with $200 \mathrm{~ms}$ are also unlikely to be the result of this mechanism. This is supported by the results of the experiment shown in figure 11. As shown in this figure maximal overdrive suppression occurs at interstimulus intervals of $400 \mathrm{~ms}$. A further decrease of the interstimulus interval results in gradual shortening of the first postpacing interval from \pm 15 to $10 \mathrm{sec}$, until abrupt 
shortening of the first postpacing occurs at an interstimulus interval of $200 \mathrm{~ms}$. This shortening is accompanied by a concomitant lengthening of the second postpacing interval (premature escape beats). As the latter are unlikely to be the result of entrance block, it is also unlikely that the less overdrive suppressed beats induced after longer interstimulus intervals than the premature escape beats are induced by entrance block. Other possible mechanisms will be discussed below.

$s \overline{\mathrm{V} . \mathrm{V}: .} \cdot \overline{\mathrm{VV}_{2} \mathrm{~s}}$

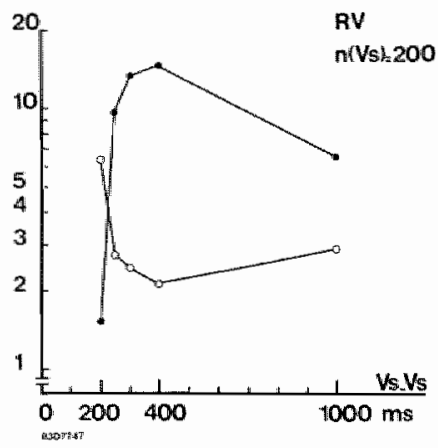

Figure 11

The concomitant occurrence of less suppressed and premature escape beats.

Arrangement of the panel is similar to figure 4.

The number of stimuli is 200 .

Interstimulus intervals are $1000,400,300,250$ and $200 \mathrm{~ms}$.

Black dots : mean of 10 first postpacing intervals.

Open dots : mean of 10 second postpacing intervals.

See text.

\section{The QRS-morphology}

The QRS-morphology before pacing and of the first and second postpacing QRS-complex were studied in 6 dogs.

The QRS-morphology of the spontaneous ventricular rhythm was always different from the conducted sinus beats before induction of complete atrioventricular block. In most

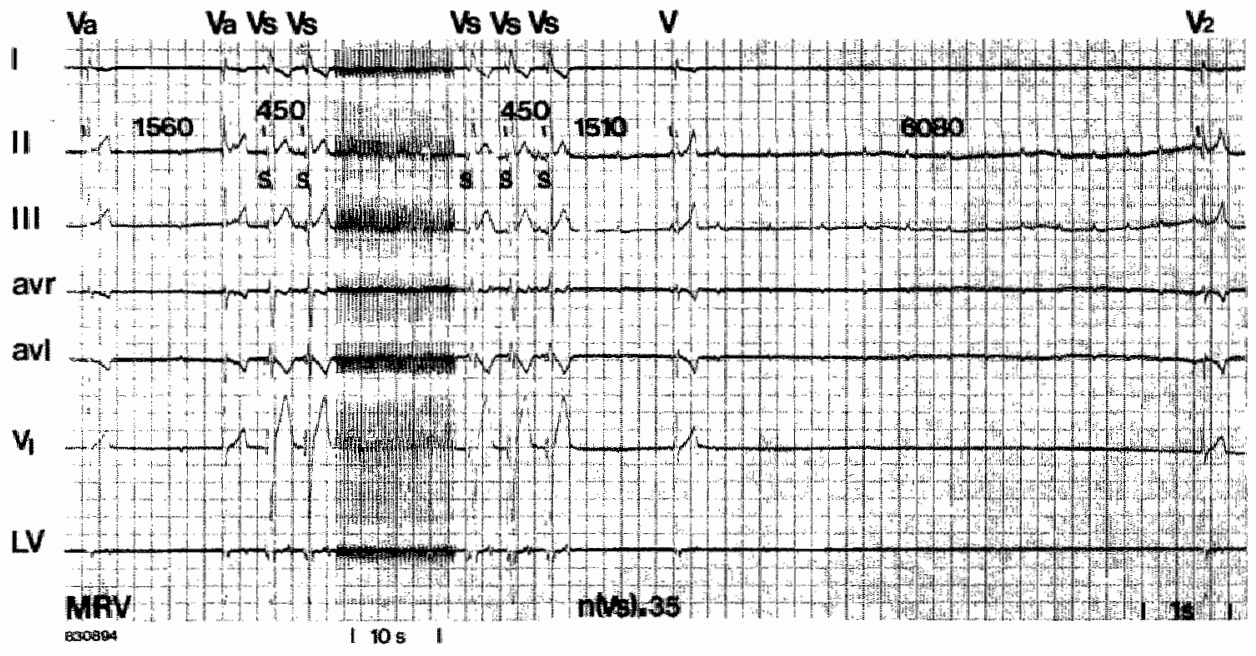

Figure 12

The sitte of origin of the first postpacing QRS-complex.

In this example a premature escape beat is induced after overdriving the spontaneous venuricular rhythm with 35 stimuli and $450 \mathrm{~ms}$ intervals.

The paper speed is decreased during the stimulation procedure. Note that the morphology of the sponaneous ventricular rthythm, the premature escape beat and the overdrive suppressed second QRS-complex are identical. See text. 
dogs more than one QRS-morphology was found (range 3 to 8, mean 5.6).

The widt th of the different QRS-complexes varied between 40 and $110 \mathrm{~ms}$, suggesting an origin within the specific ventricular conduction system. The site of origin varied from basal to apical in different animals. 16 QRS-complexes suggested a basal and 18 a more inferior origin. 29 of the QRS-complexes suggested an origin in the left ventricle.

Premature escape beats were found to have an identical QRS- morphology as the second postpacing QRS-complex and the spontaneous rhythm in 45 out of 80 stimulation trains studied (figure 12).

We also considered the possible role of formalin injection as a predillection site within the wentricles for the occurrence of premature escape beats. Such a mechanism was not supported by the observation that the furst postpacing QRS-complex in a number of cases originated in the apical part of the ventricles. Examples are shown in figure 13.
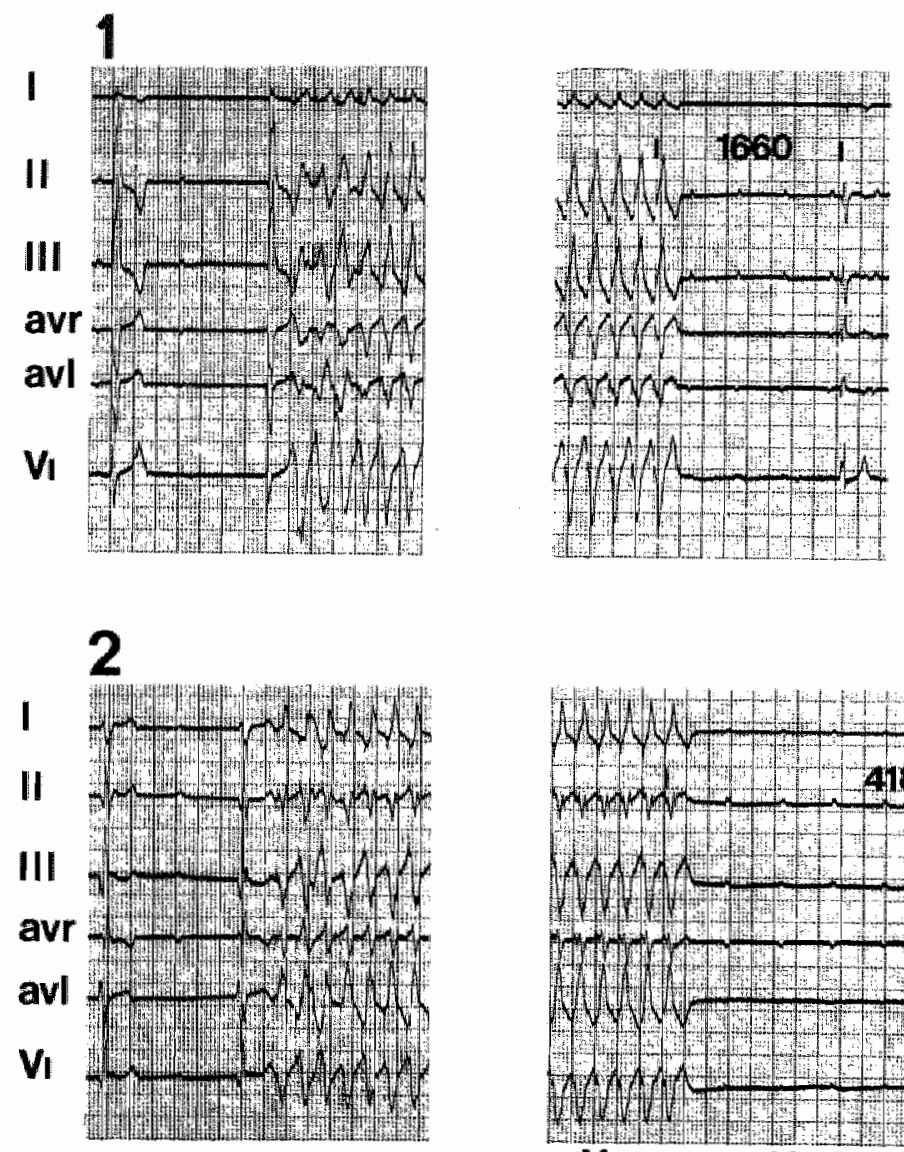

Figure 13

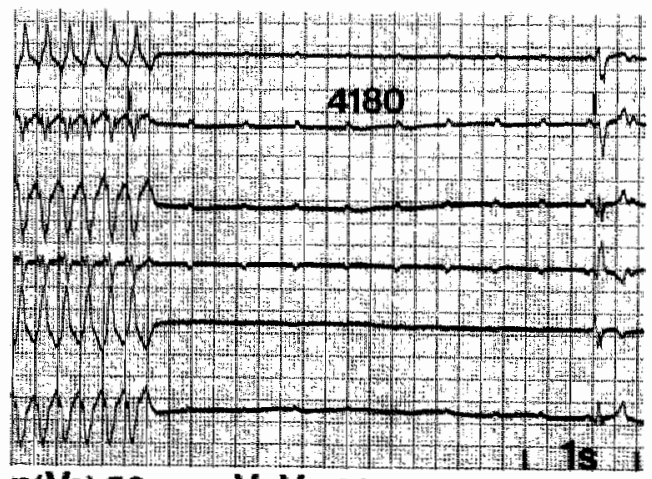

$\mathrm{n}(\mathrm{V}) \mathbf{5}) \mathbf{5 0}$

\section{Vs.Vs $200 \mathrm{MS}$}

The site of origin of the first postpacing QRS complex.

Both panels show the last prepacing QRS complexes, the pacing procedure and the first QRS-complex foilowing pacing. Paper speed is $25 \mathrm{~mm} / \mathrm{s}$ except during pacing.

In each panel the heart is overpaced with 50 stimuli and pacing intervals of $200 \mathrm{~ms}$. This results in a premature escape beat in panel 1 and in a suppressed beat in panel 2. Both responses originate in the lower part of the ventricles. 


\section{Discussion}

The data presented show that in conscious dogs with chronic atrio-ventricular block stimulation trains as short as 10 stimuli can already induce substantial changes in the duration of the intervals following overdrive of the idioventricular thythm. The amount of overdrive suppression depended on 1) the number of stimuli and 2) the interstimulus interval. Prolongation was maximal in the first postpacing interval, after which a gradual acceleration to normal values occurred in the subsequent intervals.

This behaviour is typical for overdrive suppression ${ }^{76}$. It is a characteristic of pacemaker cells in the sinus node ${ }^{41,67,82}$ and in Purkinje fibers ${ }^{121,132}$.

Its occurrence in Purkinje fibers is generally explained by 1) the activation of the sodiumpotassium pumping mechanism $40,121,2$ ) an increase in potassium conductance induced by intracellular calcium overload ${ }^{89}, 3$ ) extracellular potassium accumulation ${ }^{126}$ and 4) liberation of acetylcholine, the latter being mainly of importance in the sinus node ${ }^{129}$. The interval duration of the first postpacing interval decreased on shortening the interstimulus interval from 400 to $200 \mathrm{~ms}$. A subset of responses was recognized (premature escape beats), which behaved differently: 1) to an increase in number of stimuli 2) in their relation to the second postpacing interval, and 3) in their response to extrastimuli. Of importance is that premature escape beats were consistently induced following short and fast drives.

It is unlikely that the occurrence of premature escape beats is due to entrance block of the paced impulses into the site of impulse formation. Entrance block has been used to explain the decrease of overdrive suppression of the first postpacing interval following overdrive stimulation with increasing rates of the sinus node ${ }^{41}$. The following reasons make such a mechanism unlikely however.

1. As far as premature escape beats is concerned they were characteristically followed by an overdrive suppressed second interval. The latter interval prolonged on giving a higher number of basic stimuli whereas the first postpacing interval remained unchanged (figure $4,6,7,8$, tables 1 and 3). 2. Premature escape beats could be induced by single extrastimuli even following a stimulation train normally inducing marked overdrive suppression ( $50 \mathrm{x}$ $400 \mathrm{~ms}$ ) (figure 10 ).

Accepting that premature escape beats are based on a different mechanism than entrance block, the less suppressed QRS-complexes become also unlikely to be the result of this mechanism (figure 11).

Considering that two separate effects (overdrive suppression and premature escape beats) can be induced by overdrive stimulation it is of importance to know whether both phenomena occur concomitantly at the same site of impulse formation. Therefore the morphology of the QRS-complexes before and after stimulation was studied.

It was found that the first and second postpacing QRS-complex were identical in the majority of cases (figure 12). Also the first and second postpacing QRS-complex were frequently identical to the QRS-complexes of the spontaneous ventricular rhythm. This suggests that both mechanisms become activated at the same intraventricular site.

Because our dogs had artificial AV-block following formalin injection in the His bundle region it is conceivable that the occurrence of premature escape beats was related to this procedure. Support for such a relation would be the finding that the site of enhanced 
impulse formation was located close to the site of injection. Analysis of the QRScomplexes following stimulation revealed, however, that premature escape beats could originate distant to the site of block (figure 13). This suggests that the ability of inducing premature escape beats is a characteristic of normal ventricular pacemaker cells. It was also found that the occurrence of premature escape beats was not related to the operation procedure. Several authors have reported fast ventricular rhythms up to 1 week following the creation of AV-block ${ }^{59,128}$. This ectopic activity has been considered to be based upon abnormal automaticity or triggered activity. To exclude that this mechanism was related to our findings the experiments were always performed at least 1 week postoperatively. After this period a spontaneous ventricular rhythm had stabilized and fast spontaneous ectopic rhythms were never observed.

Furthermore, premature escape beats remained present in all dogs during the whole period of investigation (up to $1 \frac{1 / 2}{\text { year}}$.

In conclusion: overdrive stimulation of the spontaneous ventricular rhythm during chronic atrioventricular block results in overdrive suppression and in induction of premature escape beats. Both phenomena are related to the pacing rate and the number of stimuli.

Premature escape beats are characterized by :

1. an interval duration relatively unaffected by increasing the number of stimuli, until suppression occurs on reaching a critical number of stimuli.

2. its induction by short and fast drives as well as by single premature beats following regular drive.

3. an interval duration which is shorter than the second postpacing interval.

The interval duration of suppressed first postpacing QRS-complexes: 1. becomes longer on decreasing the interstimulus interval to $400 \mathrm{~ms}$, but decreases on further shortening the interstimulus interval. 2. becomes gradually longer on increasing the number of stimuli, 3. is longer than the following postpacing interval.

\section{Possible mechanisms underlying premature escape beats}

Because it is unlikely that entrance block plays a role in premature escape beats in our experiments, reentry, enhanced phase 4 depolarization and delayed afterdepolarizations have to be considered as possible underlying mechanisms. Reentry is unlikely to play a role because it does not explain the suppression of premature escape beats on increasing the number of stimuli.

Premature escape beats were characterized by a short first postpacing interval followed by an (often markedly) suppressed second postpacing interval. Apparently enhanced impulse formation was induced in the setting of normal activation of the sodium-potassium pump. Because of this, abnormal automaticity is unlikely to be the underlying mechanism. Increasing the pacing rate resulted in shortening of the first postpacing interval. Also single extrastimuli could induce early premature escape beats. Because this behaviour is compatible with triggered activity, the possibility of a delayed afterdepolarization should be considered:

Overdrive pacing results in sodium accumulation within the pacemaker cell. This favors an increase of cellular calcium through the sodium calcium exchange mechanism. In this 
setting a delayed afterdepolarization can be induced ${ }^{70,78}$, and when its amplitude becomes sufficiently high, a full depolarization may develop. Concomitantly the sodium-potassium pump becomes activated and triggered activity is stopped resulting in a long second postpacing interval ${ }^{153}$. On increasing the number of stimuli the amplitude of the delayed afterdepolarization fails to reach threshold because of hyperpolarization of the diastolic potential. This may explain why premature escape beats are less inducible at higher numbers of stimuli and after interstimulus intervals, usually resulting in the greatest amount of overdrive suppression.

This concept is supported by observations in isolated Purkinje fibers ${ }^{22}$ : Overdrive stimulation with short drives induced short (gradually prolonging) intervals followed by a delayed afterdepolarization after which the cycle length returned to control values. The less suppressed responses following pacing with $200 \mathrm{~ms}$ intervals are likely to be due to enhancement of the normally suppressed phase 4 depolarization. In the setting of a normal diastolic membrane potential the probable mechanism is an increase in sympathetic tone ${ }^{130}$. The latter may be the result of locally released catecholamines ${ }^{133}$ at the pacing site or reflex mediated by lowering of blood pressure during fast overdrive. Locally released catecholamines are unlikely to be the mechanism because the QRScomplexes following pacing originated distant to the site of pacing, and frequently in the other ventricle than the stimulated one. Support for a reflex mediated increase in sympathetic tone is the observation that the rate of the $\mathrm{P}$-waves increased during and immediately following fast overdrive (figure 2).

Another mechanism for enhanced phase 4 depolarization is partial diastolic depolarization of the ventricular pacemaker cells ${ }^{54}$. It is likely that the ventricular pacemaker cells in our dog model had a high diastolic membrane potential because the spontaneous rhythm was overdrive suppressible ${ }^{22,59}$. It is unlikely but not excluded that rapid pacing induced a decrease of the diastolic potential, as has been described in isolated Purkinje fibers ${ }^{121}$ leading to less overdrive suppression because per action potential less sodium enters the cell. Also, time for stimulation with $200 \mathrm{~ms}$ interstimulus intervals is $50 \%$ shorter than for pacing with $400 \mathrm{mns}$ intervals. This may result in a later maximal activation of the sodium potassium pump and therefore shorter first postpacing intervals.

From our experiments no definite conclusion can be drawn for the underlying mechanism of premature escape beats and the decrease in overdrive suppression following pacing with $200 \mathrm{~ms}$ pacing intervals.

The combination of programmed electrical stimulation and drugs which specifically influence cellular mechanisms of impulse formation may provide further insight in to this problem ${ }^{59}$ (chapter 7 ).

\section{Implications of our findings}

For several reasons we consider the findings described of importance. First, the concordant relation between the interstimulus interval and the first postpacing interval as has been found during digitalis intoxication (chapter 3-5) is not specific for that situation. Second, our pacing protocol evokes a response which is readily induced after short and fast drives and behaves differently from normal automaticity. This suggests a different underlying cellular mechanism. Third, the findings of premature escape beats may be similar to the frequent clinical observation of enhanced ventricular escape beats or rhythm 
following termination of spontaneously occurring tachycardia. Finally, shortening of the first postpacing interval on increasing the pacing rate has been observed following overdrive stimulation in sinus node studies ${ }^{82}$. Although entrance block can probably be more easily induced in the sinus node than in pacemaker cells within the specialized ventricular conduction system, because of the richer innervation by the autonomic nervous system and the different properties of the action potential in the former, mechanisms proposed by our results cannot be excluded. Therefore, it would be of interest to study the behaviour of the sinus node using the described pacing protocol. 


\section{Effect of lidocaine, verapamil, isoprenaline and ouabain on ventricular impulse formation following electrical stimulation.}

by

Anton PM Gorgels, MD, Bram de Wit, MD, Henriette DM Beekman, Willem RM Dassen, PhD, Hein IJ Wellens, MD Department of Cardiology Annadal Hospital MAASTRICHT The Netheriands

\section{Summary}

Idioventricular pacemakers usually become suppressed when another spontaneous or driven rhythm is above their own intrinsic rate. We have found that the interval duration of the first postpacing interval decreases at a critical pacing rate. These shorter than expected first postpacing intervals were divided in a group which behaved as classical overdrive suppression and a group which behaved differently to programmed electrical stimulation. The latter QRS-complexes were termed premature escape beats. Premature escape beats have a shorter first postpacing interval than its second one. They are induced by fast and short overdrive trains and by extrastimuli following regular pacing.

In the present study we tested the hypothesis that premature escape beats are based upon triggered activity. For this purpose programmed electrical stimulation of the ventricle was done in conscious dogs with chronic complete atrioventricular block in combination with drugs which are known to influence triggered activity and overdrive suppression. The stimulation protocol included regular pacing using 50 stimuli and interstimulus intervals of $1000,800,400$ and $200 \mathrm{~ms}$. The protocol was repeated after the intravenous administration of lidocaîne, verapamil, isoprenaline and ouabain.

The spontaneous ventricular rhythm was slowed by lidocaine, accelerated by isoprenaline and verapamil administration and largelly unaffected by ouabain in a non-toxic dose. Overdrive suppression increased during lidocaine, and toxic amounts of ouabain and decreased following verapamil. Isoprenaline decreased overdrive suppression when interstimulus intervals of 800 and $400 \mathrm{~ms}$ were used but after $200 \mathrm{~ms}$ overdrive suppression of the second interval increased. Lidocaine and verapamil decreased the number of premature escape beats and lengthened their interval duration, whereas oubain and isoprenaline had the opposite effect.

It is concluded that triggered activity is the most likely mechanis of premature escape beats.

Idioventricular pacemakers usually become suppressed when a spontaneous or driven rhythm is faster than their own intrinsic rate ${ }^{72,75,76,77,127}$. This phenomenon is termed overdrive suppression. We have found that the first postpacing interval decreases at a critical pacing rate (chapter 6). These QRS-complexes were divided in a group which behaved as classical overdrive suppression and an other group which behaved differently to programmed electrical stimulation. The latter QRS-complexes were termed premature escape beats. Premature escape beats are characterized by first postpacing intervals which are 1) not influenced by increasing the number of stimuli until marked overdrive 
suppression occurrs at a critical number of stimuli, 2) followed by a longer second postpacing interval, 3) already inducible by single extrastimuli during basic pacing. We hypothesized that triggered activity was the underlying mechanism for premature escape beats. To test this hypothesis programmed electrical stimulation was combined with drugs known to influence cellular mechanisms of impulse formation. Lidocaine was selected because it has a suppressing effect both on triggered activity ${ }^{29,99}$ and phase 4 depolarization ${ }^{23}$, whereas it suppresses abnormal automaticity only in high concentrations (M.R. Rosen, personal communication). Verapamil has a (use dependent) suppressing effect on triggered actiwity ${ }^{7,979}$. Ouabain ${ }^{17,18,35,18,87,97}$ and isoprenaline ${ }^{153}$ induce triggered activity and the latter also enhances phase 4 depolarization ${ }^{122}$.

\section{Methods}

The experiments were performed in 4 dogs with chronic complete atrioventricular block. Through a right thoracotomy unipolar electrodes were fixed intramurally into the myocardium of the basal free wall of the right ventricle.

Atrioventricular block was made by injecting formalin $37 \%$ in the region of the His bundle 114. The electrode catheters were exteriorized through the skin of the neck. After a recovery period of at least one week the experiments were performed without any premedication. This one week periode of rest was necessary to avoid episodes of spontaneous ventricular tachycardias during the study which are known to occur in the early postoperative period ${ }^{59,128}$.

Six electrocardiographic leads (I, II, III, AVR, AVL and V) and one epicardial lead were simultaneously registered on a ink-iet recorder (with a paper speed of $100 \mathrm{~mm} / \mathrm{s}$ ) and stored on tape. Electrical stimulation was done with a programmable stimulator having a synchronizing circuit. Unipolar stimuli were given with a stimulus strength of twice the diastolic threshold. Between different stimulation trains two to 5 minutes was waited to allow the ventricular rate to recover completely.

\section{The pacing protocol}

The spontaneous ventricular thythm was overpaced 5 to 12 times with 50 stimuli and pacing intervals of $1000,800,400$ and $200 \mathrm{~ms}$. Thereafter a drug was administered and the pacing protocol repeated.

\section{Mode of drug administration}

After a drug study several days lapsed to allow the drug to wash out completely before another drug was studied. All drugs were intravenously injected. Lidocaine was given in all dogs as a bolus of $3 \mathrm{mg} / \mathrm{kg}$ in 2 minutes followed by a continuous infusion of 100 $\mu \mathrm{g} / \mathrm{kg} / \mathrm{min}$.

Verapamil was also administered in all dogs: an injection of $0.4 \mathrm{mg} / \mathrm{kg}$ ower 10 minutes was given, followed by an infusion of $0.6 \mathrm{mg} / \mathrm{kg} / \mathrm{hour}$.

Ouabain was given in one dog as a bolus injection of $0.5 \mathrm{mg}$ resulting in ventricular tachycardia. In two other dogs a non-toxic regimen was followed: a bolus injection of 20 $\mu \mathrm{g} / \mathrm{kg}$ was given, followed by an infusion of $0.036 \mu \mathrm{g} / \mathrm{kg} / \mathrm{min}$.

Isoprenaline was administered in a dose of 3-3,6 $\mu \mathrm{g} / \mathrm{min}$. 


\section{Analysis of data}

Between consecutive stimulation trains enough time was allowed ( $2-5$ minutes) for the ventricular rhythm to regain its prepacing rate. Therefore it was possible to use the last prepacing interval for determining the rate of the spontaneous ventricular thythm.

The first and the second postpacing intervals were used to identify premature escape beats. Premature escape beats were present when the first postpacing interval was shorter than the second one.

\section{Results}

The spontaneous ventricular rhythm

The effect of the different drugs on the spontaneous ventricular rhythm is listed in table 1. Lidocaine slowed the spontaneous ventricular rate, verapamil and isoprenaline had an accelerating effect, ouabain in a non-toxic dose did not affect the spontaneous thythm.

\section{Table 1.}

The effect of lidocaine, verapamil, isoprenaline and oubatin on the spontancous rhythm.

\begin{tabular}{lcccl}
\hline & $\mathrm{V}_{\mathrm{a}}-\mathrm{V}_{\mathrm{a}}$ & $\mathrm{SD}$ & $\mathrm{n}$ & $\mathrm{P}$ (control $\mathrm{s}$ drug) \\
control & 1374 & 168 & 160 & $<0.001$ \\
lido & 1589 & 360 & 162 & \\
control & 1320 & 121 & 88 & $<0.001$ \\
verapamil & 1198 & 127 & 83 & \\
control & 1412 & 105 & 160 & $<0.001$ \\
isoprenaline & 942 & 83 & 114 & \\
control & 1417 & 140 & 80 & $<0.2(\mathrm{n} . \mathrm{s})$ \\
ouabain & 1450 & 130 & 80 & \\
\hline
\end{tabular}

Abbreviations: $\mathrm{SD}=$ standard deviation. $n=$ number of spontaneous intervals measured. $\mathrm{n} . \mathrm{s}=$ not significant (unpaired student's [-test)

\section{The effect of overdrive stimulation}

Figure 1 and table 2 illustrate the induction of premature escape beats. Maximal overdrive suppression was attained following interstimulus intervals of $400 \mathrm{~ms}$. Decreasing the interstimulus interval to $200 \mathrm{~ms}$ always resulted in a shorter first postpacing interwal. When the second postpacing interval was shorter than the first one it was termed a premature escape beat (chapter 6 ).

\section{The effect of the different drugs on overdrive stimulation}

The number and interval duration of premature escape beats were significantly influenced by the different drugs.

Lidocaine and verapamil decreased the number of premature escape beats increased their interval duration whereas ouabain and isoprenaline had the opposite effect. 

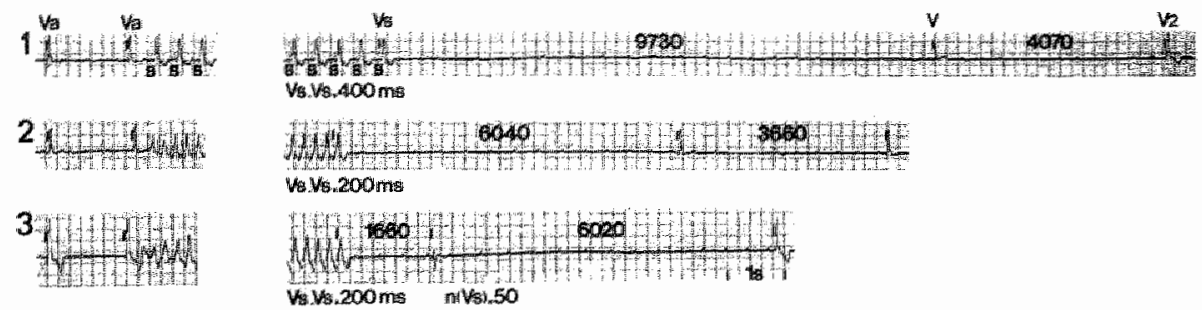

Fingure 1

The effect of overdrive stimulation on the spontaneous ventricular thythm.

Three recordings illustrating the induction of overdrive suppression (panel 1), less overdrive suppression on increasing the pacing rate (panel 2) and premature escape beats (panel 3). Regular pacing is done with 50 stimuli using $400 \mathrm{~ms}$ (panel 1) and 200 ms intervals (panels 2 and 3). Note that the first postpacing interval in panel 2 is shorter that in panel 1 and that the first postpacing interwal is longer than the second one. In panel 3 the first postpacing interval is also shorter than in panel 1 but the second interval is longer than the first one.

Table 2

The first postpacing interval in relation to the interstimulus interval and the second postpacing interval.

$\begin{array}{lllllll}\mathrm{V}_{\mathrm{it}}-\mathrm{V}_{\mathrm{s}}: 1314= & 162 & & & & & \\ \mathrm{n}\left(\mathrm{V}_{\mathrm{s}}\right) & \mathrm{V}_{\mathrm{s}}-\mathrm{V}_{\mathrm{s}} & \mathrm{V}_{\mathrm{s}}-\mathrm{V} & \mathrm{SD} & \mathrm{V}-\mathrm{V}_{2} & \mathrm{SD} & \mathrm{n} \\ 50 & 1000 & 2893 & 1865 & 2033 & 765 & 59 \\ & 800 & 3925 & 2673 & 2291 & 681 & 59 \\ & 400 & 7359 & 2398 & 3143 & 630 & 60 \\ & 200 & 1507^{\star} & 188 & 3625 & 2298 & 29 \\ & & 5027 & 1157 & 3096 & 675 & 31\end{array}$

Combined data from six dogs which include the four dogs used during the drug studies. All measurements are expressed in milliseconds. For abbreviations see text.r.

SD : standard deviation.

$\mathrm{n}$ : number of stimulation trains.

* : premature escape beats

\section{Lidocaine}

The effect of lidocaine on premature escape beats is illustrated in figure 2. Panel 1 shows the induction of a premature escape beat. This was observed in all 42 stimulation trains in two dogs. In the 19 stimulation trains in the two remaining dogs overdrive suppression was observed (panel 2). Lidocaine increased the interval duration of overdrive suppressed beats substantially, and the second postpacing interval as well (panel 4).

The effect on the premature escape beats was different. Whereas the interval duration increased only slightly, the second postpacing interval became markedly prolonged. However, the number of premature escape beats decreased significantly (from all 42 during the control experiment to 17 out of 44 during lidocaine administration).

Figure 3 and table 3 show the combined results from the different experiments. In the control experiment overdrive suppression occurred between pacing intervals of 1000 and $400 \mathrm{~ms}$. In two dogs premature escape beats occurred following interstimulus intervals of $200 \mathrm{~ms}$, and in the other two dogs overdrive suppression. During lidocaine administration overdrive suppression increased. In contrast the premature escape interval became only slightly longer $(1661 \pm 143 \mathrm{~ms}$ versus $1787 \pm 172 \mathrm{~ms}, \mathrm{p}<0.001)$ but less premature escape beats were induced than under control conditions. 


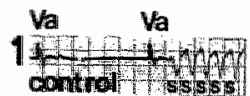

2 cintrat $-M$
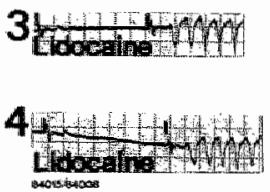

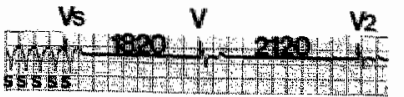

n

42

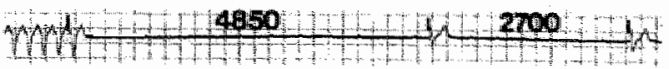

19

M

17

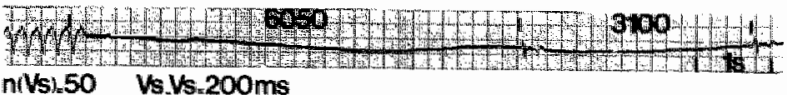

44

Figure 2

The effect of lidocaine on overdrive suppression and premature escape beats.

The last prepacing interval, the pacing procedure and the first and second postpacing interval are shown. Pacing is done with 50 stimuli and interstimulus intervals of $200 \mathrm{~ms}$.

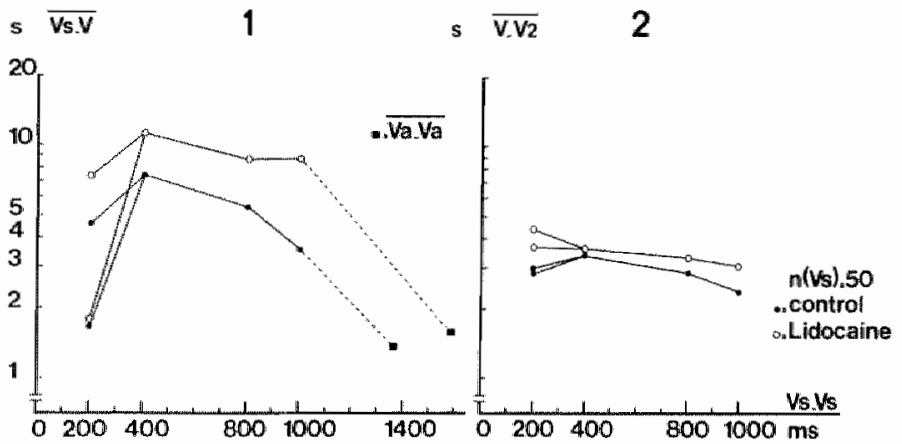

Figure 3

The effect of lidocaine on overdrive stimulation.

Graph showing the combined results from 4 dogs. The postpacing intervals are plotted as a function of the interstimulus interval. The vertical axis has a logarithmie scale. The feft panel shows the duration of the first pacing interval and the right panel the duration of the second one.

Squares: mean of the last prepacing interval.

Black dots: mean of the postpacing intervals during the control experiments.

Open dots: mean of the postpacing intervals during lidocalime administration.

\section{Verapamil}

Verapamil accelerated the spontaneous ventricular rhythm (table 1). Following overdrive with interstimulus intervals of $400 \mathrm{~ms}$ the amount of overdrive suppression was significantly less than in the control experiments (figure 4, table 4).

After pacing intervals of $200 \mathrm{~ms}$ overdrive suppression also decreased. In contrast the interval duration of the premature escape beats increased (panel 1 and 2 of figure 4$)(1618$ $\pm 189 \mathrm{~ms}, \mathrm{n}=24$ versus $2526 \pm 474 \mathrm{~ms}, \mathrm{n} \pm 5, \mathrm{p}<0.001)$ and also the number of premature escape beats decreased in comparison to control $(5 / 41$ versus $24 / 44, p<0.001)$. 

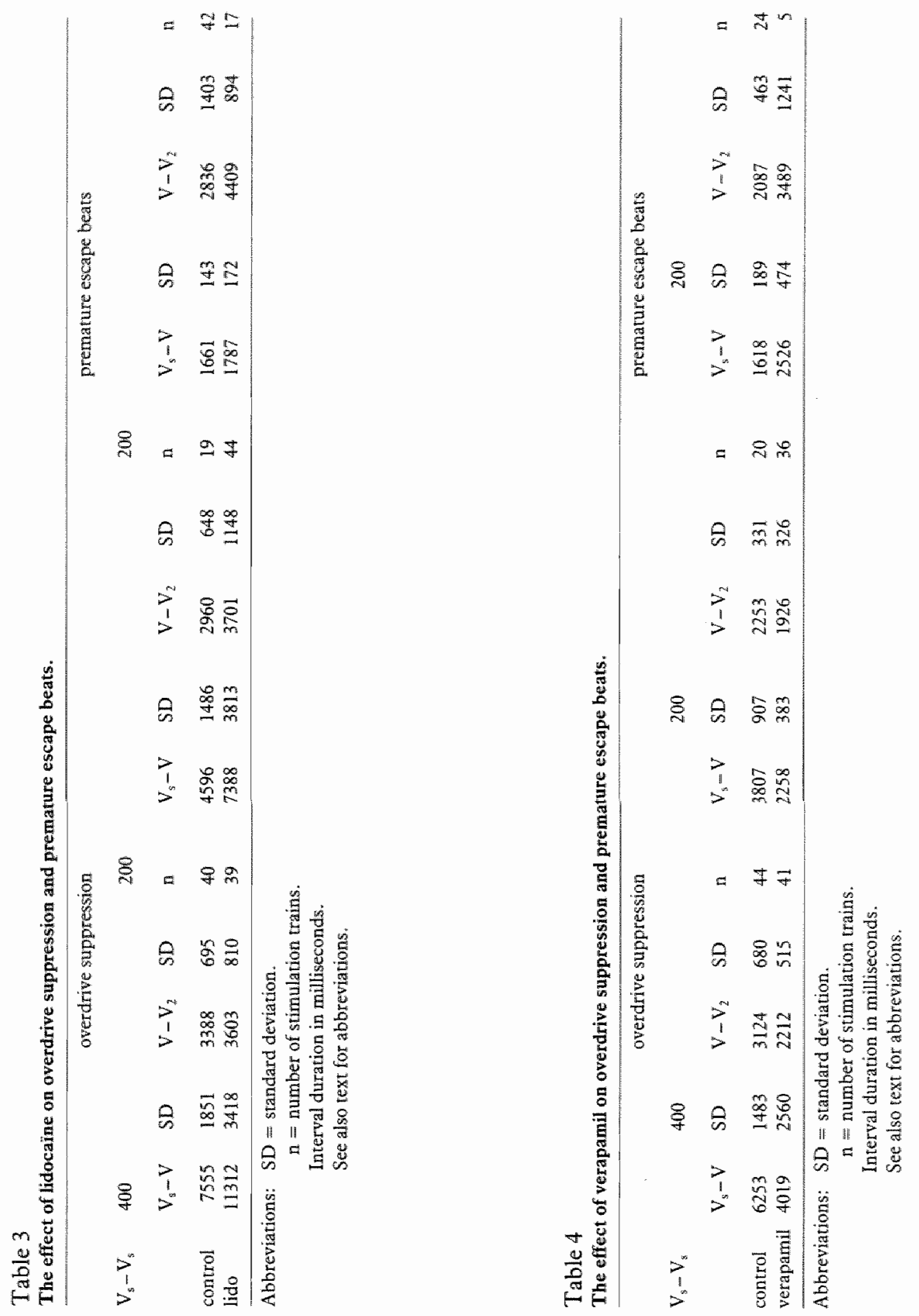

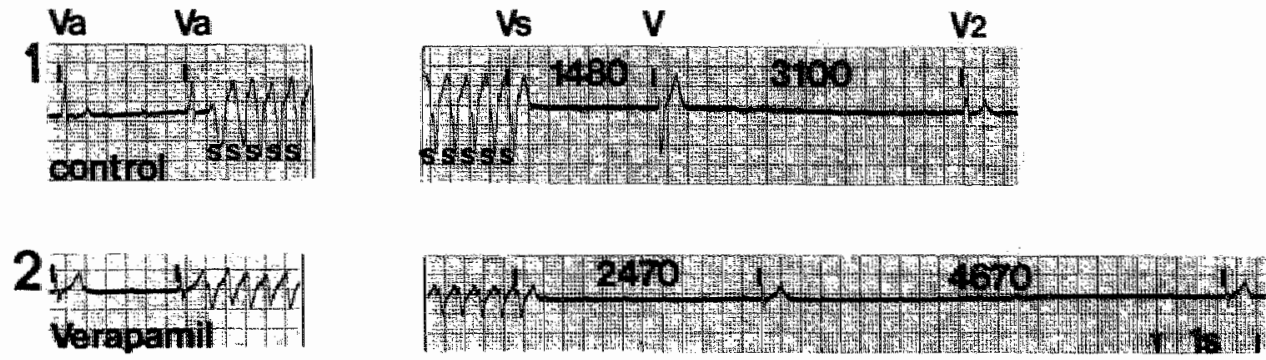

Vs.Vs.200ms

3

s. $\overline{v s . V}$

s $\overline{\mathbf{V} / 2}$

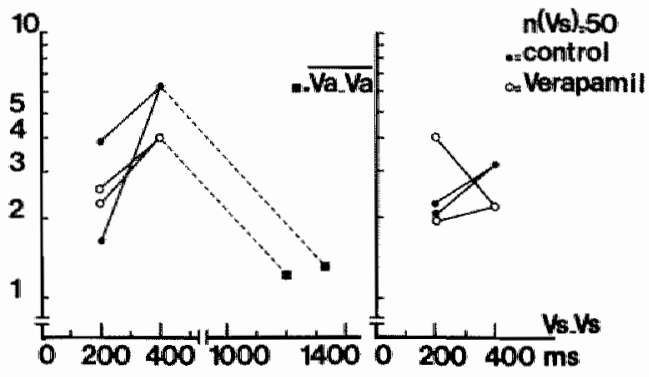

Figure 4

The effect of verapamil on overdrive stimulation.

Panels $1-3$ are arranged as in figures 2 and 3 . Note the increase in interval duration of the premature escape beats during verapamil administration. Panel 3 shows the combined data from 4 dogs. See text.

\section{Isoprenalline}

Isoprenaline markedly accelerated the spontaneous ventricular rhythm (table 1). This prohibited overdrive stimulation with $1000 \mathrm{~ms}$ intervals. Also the duration of the first and second postpacing interval was less but only following interstimulus intervals of 800 and $400 \mathrm{~ms}$ (figure 5, panel 1 and 3, figure 6). After pacing intervals of $200 \mathrm{~ms}$ the first postpacing QRS-complex was a premature escape beat in all cases (38/38), whereas in the control experiments this was only observed after 19 out of 40 stimulation trains (figure 5 , panel 2 and 4).

Also the interval duration of the premature escape beats was shorter than under control conditions $(1763 \pm 455 \mathrm{~ms}, \mathrm{n}=19$, versus $1441 \pm 299 \mathrm{~ms}, \mathrm{n}=38, \mathrm{p}<0.001)$.

Interestingly the second postpacing interval became longer than in the control experiments ( $2649 \pm 946 \mathrm{~ms}, \mathrm{n}=19$, versus $3548 \pm 2867 \mathrm{~ms}, \mathrm{n}=38, \mathrm{p}<0.001$ )(figure 5 , panel 4).

Figure 6 shows the combined data. All data are listed in table 5. 

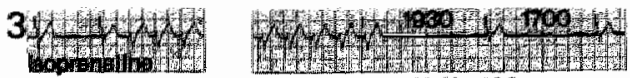

Vs.Vs.400ms
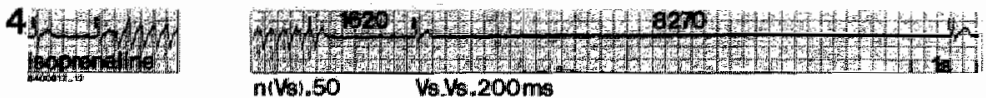

Figure 5

The effect of isoprenaline on overdrive stimulation.

Panel 1 and 2 are recorded during the control experiment. Panel 1 shows overdrive suppression following 50 stimuli and intervals of $400 \mathrm{~ms}$. Panel 2 shows a less overdrive suppressed QRS-complex. Panel 3 and 4 were recorded during isoprenaline administration. Pancl 3 shows a marked decrease of overdrive suppression following 50 stimuli with intervals of $400 \mathrm{~ms}$. Panel 4 shows the induction of a premature escape beat followed by marked overdrive suppression.

12

s $\overline{v s . V}$

$s \overline{v \sqrt{ } 2}$

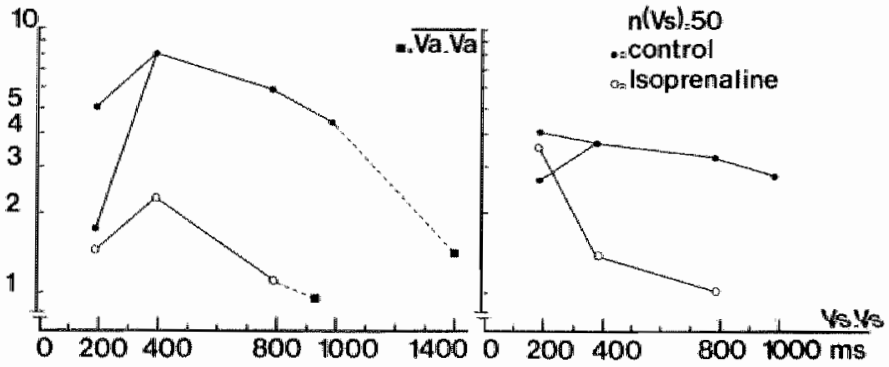

Figure 6

The effect of isoprenaline on overdrive stimulation.

The combined data from 4 dogs are arranged as in figure 3 . Note the marked decrease of overd rive suppression during isoprenaline administration and the induction of premature escape beats. The second postpacing interval after pacing intervals of $200 \mathrm{~ms}$ is longer than the second postpacing intervals of the premature escape beats during the control experiments.

\section{Ouabain}

Ouabain administration in a non toxic dose did not affect the spontaneous ventricular rhythm (table 1). Also the effect on overdrive suppression was small (table 6, figure 7). The following effects on premature escape beats were observed: 1.

Premature escape beats could be induced after non-toxic amounts of ouabain (figure 7) in dogs in whom no premature escape beats were induced in the control experiment after pacing with 50 stimuli and interstimulus intervals of $200 \mathrm{~ms}$ (figure 7, panel 1 and 3). One and a half hour after the start of ouabain administration premature escape beats were observed following all stimulation trains (figure 7, panel 2 and 3, table 6). 

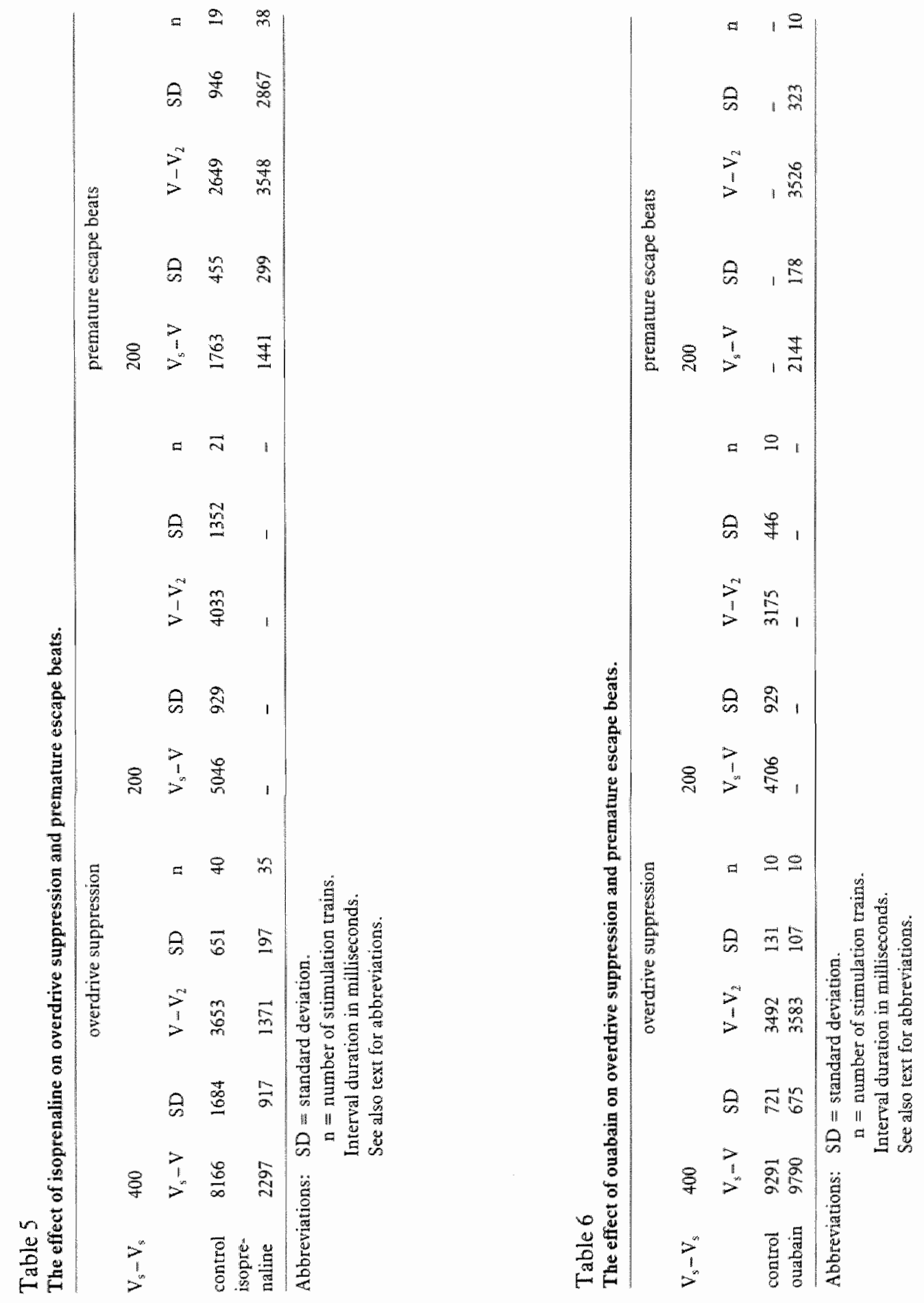

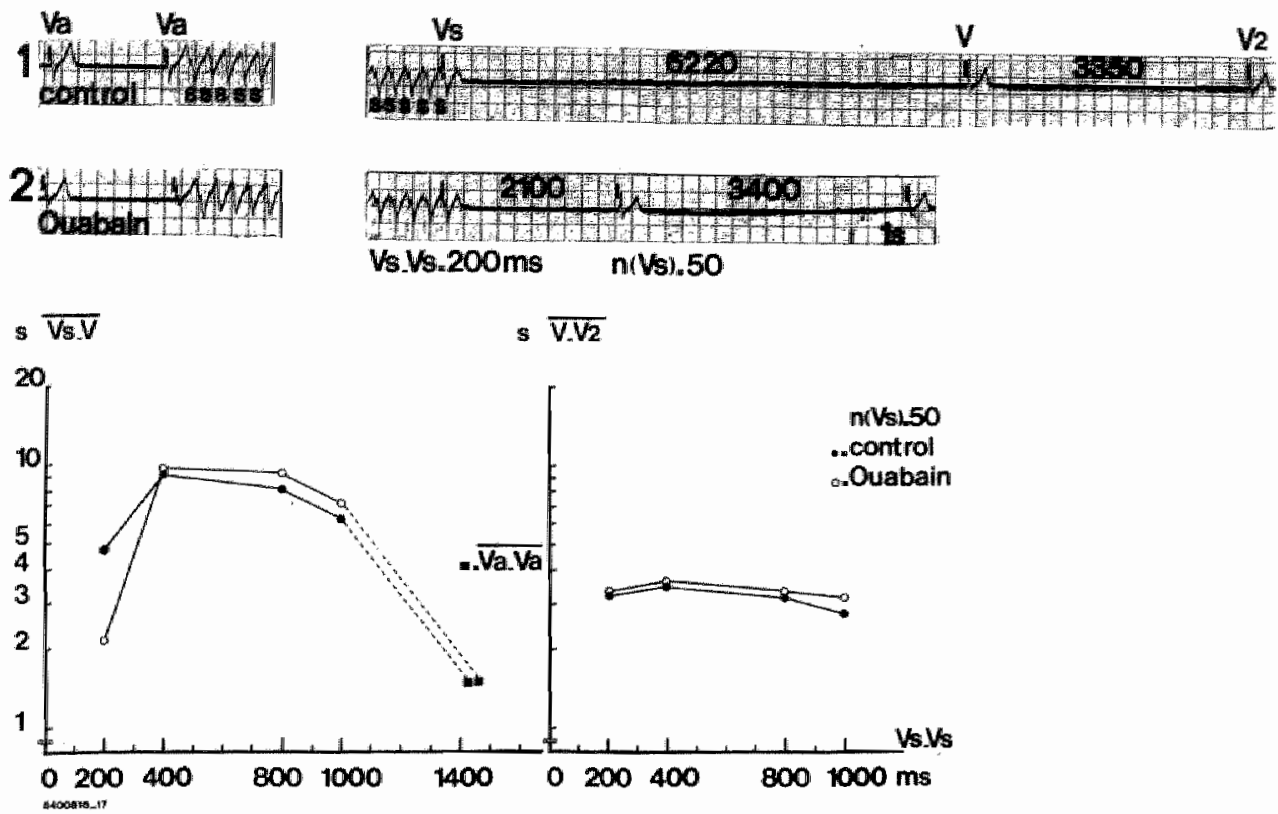

Figure 7

The effect of a non-toxic dose of ouabain on overdrive stimulation.

Arrangement of panels as in figures 2 and 3. Panel 1 shows overdrive suppression during the control experiment. Panel 2 shows the induction of a premature escape beat during a noti-toxic dose of oubain.

Panel 3 shows the combined data during ouabain administration from two dogs who did not show premature escape beats in the control experinents. During ouabain administration only premature escape beats were induced.

2.

In dogs showing premature escape beats following interstimulus intervals of $200 \mathrm{~ms}$ in the control experiment the interval duration of these responses became shorter at higher levels of digitalis toxicity. This is shown in figure 8. Overdrive stimulation was repeated at decreasing levels of digitalis toxicity to non-toxic levels. The curve representing the relation between the pacing rate and the first postpacing interval gradually shifted to values obtained before ouabain administration.

When no premature escape beats $\left(V_{s}-V_{s}>500 \mathrm{~ms}\right.$ ) were induced overdrive suppression was observed. Interestingly, it was found that overdrive suppression was most pronounced at higher levels of toxicity.

3.

At increasing levels of digitalis intoxication not only the first postpacing QRS-complex was a premature escape beat but they were also observed in the subsequent intervals: The relation of the one interval to its following interval became reversed to the normal behaviour of overdrive suppression i.e. the interval was shorter than its following one. By this, the interwal showing most overdrive suppression became more and more delayed at increasing levels of digitalis toxicity. While concomitantly the duration of the intervals became increasingly shorter, this finally resulted in sustained ventricular tachycardia (figure 9). 
5 VS.V

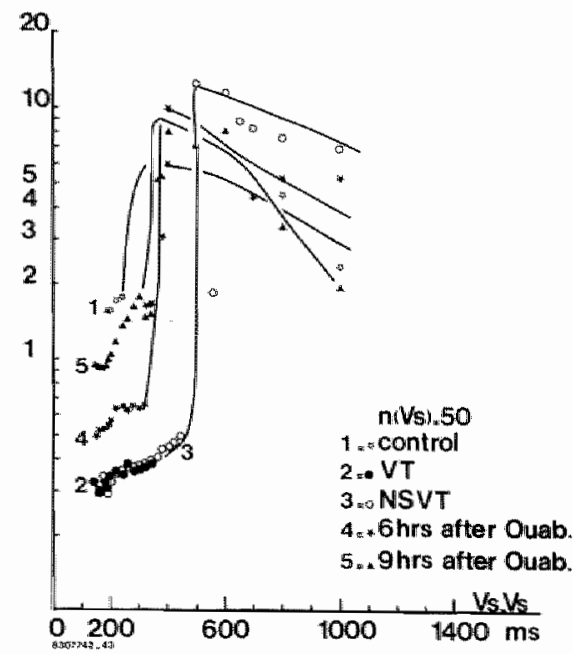

Figure 8

The effect of overdrive stimulation at different levels of ouabain toxicity.

Overdrive stimulation is repeated at different levels of oubain toxicity:

1: control

2: VT: sustained ventricular tachycardia,

3: NSVT: non-sustained ventricular tachycardia.

4: 6 hours after ouabain administration.

5: 9 hours after ouabain administration.

The first postpacing interval is plotted on a logarithmic scale as a function of the interstimulus interwal. Pacing at intervals from 1000 to $400 \mathrm{~ms}$ results in overdrive suppression which is more pronounced at higher levels of toxicity. Berween interstimulus intervals of 400 and 200 ms premarure escape beats are observed, the interval duration gradually increasing from curve 2 to curve 5 . Curve 5 was slightly below control value. 

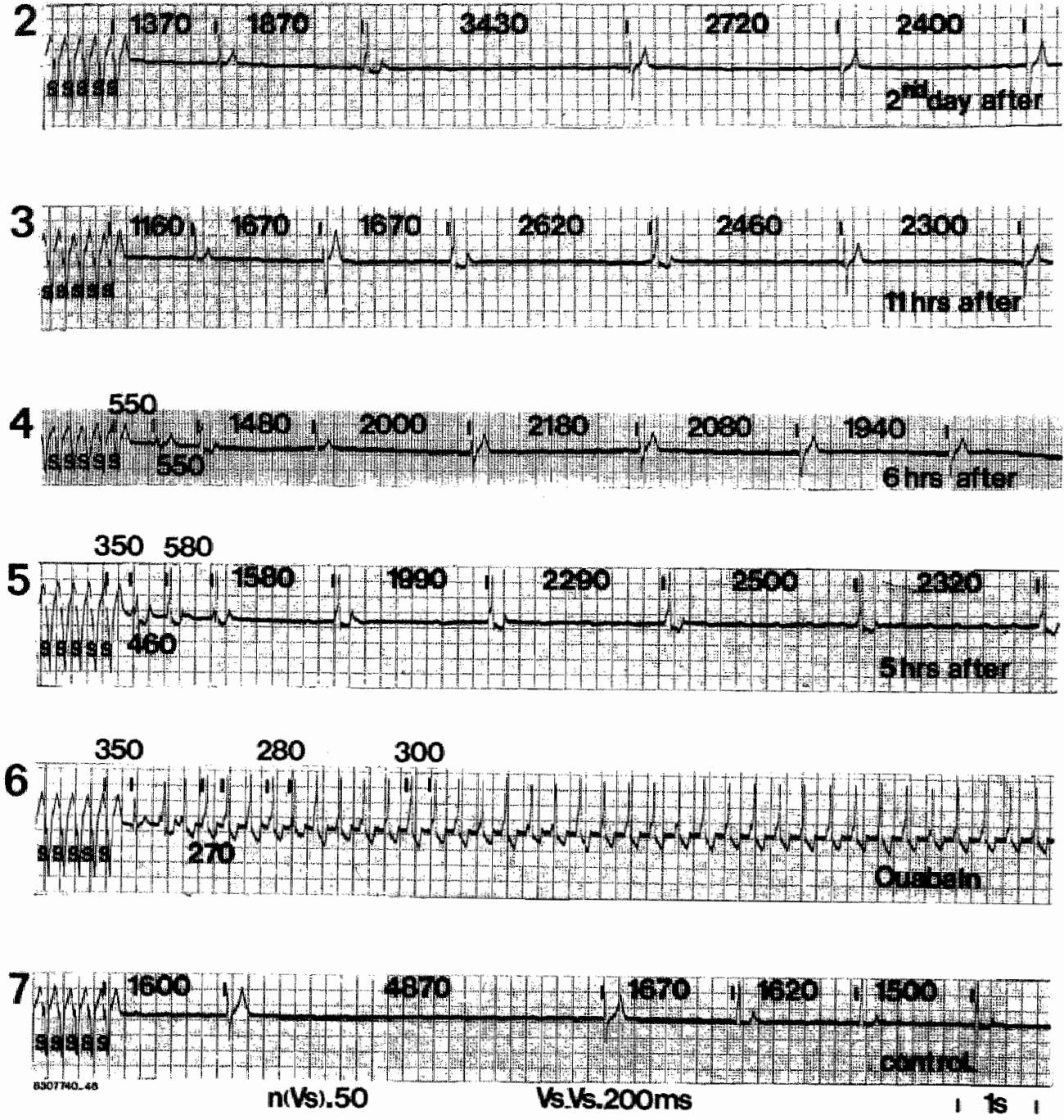

Figure 9

The effect of overdrive stimulation at different leveis of ouabain toxicity.

The panels are arranged from control to high lewels of digitalis toxicity from 5 days after to immediately after bolus injection). All recordings are from pacing with 50 stimuli and pacing intervals of $200 \mathrm{~ms}$. From panel 1 to 6 ouabain toxicity increases from values comparable to control to sustained ventricular tachycardia.

Note that at increasing levels of toxicity premature escape beats also occur in the intervals following the first postpacing interval. This results in an increasing delay in appearence of the longest postpacing interval. Also the interval length of premature escape beats shortens at higher levels of digitalis toxicity. 


\section{Discussion}

During the control experiments the first and second postpacing interval behaved as previously described (chapter 6). Maximal overdrive suppression was observed after interstimulus intervals of $400 \mathrm{~ms}$. On increasing the pacing rate the first postpacing interval decreased. When the first postpacing interval was shorter than the second one it was called a premature escape beat. The different behaviour of premature escape beats to programmed electrical stimulation suggests a different mechanism than the one underlying overdrive suppression.

We considered premature escape beats to be due to triggered activity.

Therefore overdrive stimulation was performed in combination with drugs which are known to unfluence one or both mechanisms. The results of the effect of the different drugs on premature escape beats are summarized in table 7 .

Table 7

\begin{tabular}{lllll} 
& NA & AA & DAD & PEB \\
\hline lidocaïne & $\downarrow$ & - & $\downarrow$ & $\downarrow$ \\
verapamil & $\uparrow$ or $\downarrow$ & $\uparrow$ or $\downarrow$ & $\downarrow$ & $\downarrow$ \\
isoprenaline & $\uparrow$ & $\uparrow$ & $\uparrow$ & $\uparrow$ \\
ouabain (toxic) & $\downarrow$ & $\uparrow$ or $\downarrow$ & $\uparrow$ & $\uparrow$ \\
\hline
\end{tabular}

NA : normal automaticity

A.A : abnormal automaticity

DAD : delayed afterdepolarization

PEB : premature escape beat

The effect of different drugs on cellular mechanisms of impulse formation and premature escape beats. Note that the effect of drugs on premature escape beats fits best with triggered activity.

As expected overdrive suppression increased after lidocaine and also after toxic amounts of ouabain. For the ouabain induced increase in overdrive suppression an increase in vagal tone is unlikely to be the mechanism as this does not appear to play a role in overdrive suppression in the ventricles ${ }^{129}$. Moreover overdrive suppression is also increased in isolated Purkinje fibers exposed to toxic amounts of digitalis, suggesting a cellular mechanism ${ }^{32}$. This mechanism may be the ouabain induced intracellular calcium accumulation as this is known to cause hyperpolarization by increasing potassium conductance ${ }^{89}$. Another mechanism may be the ouabain induced inhibition of the sodium-potassium ATP-ase. This increases the intracellular sodium and extracellular potassium content. It is conceivable that this can lead to a net enhanced activation of the sodium-potassium pump. Finally, digoxin is known to inhibit the sodium pacemaker current (M.R. Rosen, personal communication). 
Isoprenaline decreased overdrive suppression following interstimulus intervals of 800 and $400 \mathrm{~ms}$. This is expected as catecholamines are known to enhance diastolic depolarization 49,92. After pacing with intervals of $200 \mathrm{~ms}$ premature escape beats were observed followed by a pause which was longer than during the control experiments. This paradoxical finding of prolonged cardiac standstill during isoprenaline infusion may have different underlying mechanisms.

First the sodium potassium pump is activated by catecholamines, resulting in hyperpolarization of the diastolic membrane potential ${ }^{7,15,124}$. Furthermore calcium influx is activated, resulting in intracellular calcium accumulation inhibiting diastolic depolarization ${ }^{89}$. Support for the latter consideration is found in a recent report on the effect of norepinephrine on delayed afterdepolarizations and triggered activity in isolated Purkinje fibers ${ }^{73}$. In this study it was found that high calcium concentrations prohibited the norepinephrine induced enhanced automaticity. In this setting following rapid stimulation delayed afterdepolarizations and triggered activity occurred being followed by a long pause.

The observed decrease in overdrive suppression during verapamil administration may be the result of the increase in sympathetic tone ${ }^{59,74}$ because of peripheral vasodilatation and a possible decrease of myocardial contractility. Also a direct cellular effect may be responsible, i.e. a verapamil induced reduction of the intracellular calcium content ${ }^{58,89}$.

The overdrive suppressed responses after pacing with intervals of $200 \mathrm{~ms}$ behaved similarly to lidocaïne and verapamil as did overdrive suppression following $400 \mathrm{~ms}$ pacing intervals. Previously we considered these beats to be caused by enhanced phase 4 depolarization either because of an increased sympathetic tone during rapid stimulation, or because of a partial diastolic depolarization ${ }^{121}$ resulting in less sodium entering the cell per upstroke and less activation of the sodium-potassium pump ${ }^{22}$. From our experiments no conclusion could be drawn which mechanism is most likely.

We have found that premature escape beats have a direct linear relation to the pacing interval and could be induced by a single extrastimulus following basic pacing (chapter 6). These findings are suggestive for triggered activity as the underlying mechanism ${ }^{32,87}$. Increasing the number of stimuli inhibited the induction of premature escape beats, a phenomenon which has also been described in arrhythmias, due to triggered activity ${ }^{13 !}$. Also the effect of drugs points to triggered activity (table 7). Lidocaine hardly affected the interval duration of premature escape beats but substantially decreased the number induced. This is in agreement with the finding that lidocaine reduces the amplitude of delayed afterdepolarizations ${ }^{29,99}$.

It makes abnormal automaticity unlikely because this mechanism is not influenced by this drug in the dose used in our experiments ${ }^{59}$.

Lidacaine in high concentrations is known to suppress also abnormal automaticity. Although no plasma levels were determined in our experiments it is very unlikely that abnormally high concentrations were attained. In none of the experiments clinical signs of toxicity were observed and the dose we used was comparable to clinical dose regimens. Verapamil also reduced the number of early premature escape beats and increased their 
interval duration. As verapamil inhibits calcium entrance into the cell and delayed afterdepolarizations occur in the setting of increased intracellular calcium accumulation 70,78 our findings can be explained by delayed afterdepolarizations. Calcium accumulation within the cell is induced by catecholamines ${ }^{95}$ and digitalis derivatives ${ }^{89}$. In our experiments these drugs increased the number of premature escape beats and decreased their interval duration.

Increasing levels of ouabain also induced premature escape beats in the intervals after to the first postpacing interval, untill after the longest interval the rate gradually increased to control llevels (figure 9). These findings are similar to observations in isolated Purkinje fibers after short drives, which thave been called biphasic responses ${ }^{22}$. Here, delayed afterdepolarizations occurred after the last action potential and preceding the longest post-drive cycle.

In conclusion we consider our findings very suggestive for triggered activity as the mechanism for premature escape beats. The less suppressed responses after pacing with $200 \mathrm{~ms}$ intervals behave similarly as overdrive suppression and are probably the result of enhancement of the normally suppressed phase 4 depolarization.

\section{The value of our observations for further studies}

In the human heart and experimentally probably the best studied arrhythmogenic mechanism is reentry. Programmed electrical stimulation has provided detailed information on its mechanism ', modes of initiation and termination ${ }^{68,146}$ and ways of treatment ${ }^{25,84,144}$ of arrhythmias based upon this mechanism.

The role of triggered activity in clinical arrhythmias has not been well established ${ }^{9}$. In contrast, in different tissues of the animal heart it has been found to be an important arrhythmogenic mechanism. Delayed afterdepolarizations can occur in normal cardiac tissues, such as coronary sinus ${ }^{150,153}$ atrioventricular valve ${ }^{151}$ and in ventricular specialized conducting tissue after exposure to altered ionic conditions ${ }^{17}$, toxic concentrations of digitalis $17,18,32,35,48,87,97,99$ and after myocardial infarction ${ }^{22}$. Therefore, triggered activity is likely to be an important mechanism for several clinically occurring arrhythmias. These arrhythmias are however frequently difficult to study clinically because its induction and termination by programmed electrical stimulation is not as reproducible as in reentrant tachycardia, and because the clinical setting in which triggered activity occurs is frequently not ideal for systematic study.

Especially because of these considerations the conscious dog model with formalin induced AV-block can be of value to study triggered activity in the intact heart under different pathological circumstances and the effect of pharmacological interventions on arrhythmias based upon this mechanism. 



\section{Summary}

This thesis reports on the effect of programmed electrical stimulation of the heart on ventricular rhythm in conscious dogs with chronic complete atrioventricular block with and without digitalis intoxication.

Already before the Christian era the foxglove was known for its benificial medicinal qualities. In 1542 the botanist and physician Leonhard Fuchs gave this plant its scientific name "digitalis" 39 .

Since Withering ${ }^{158}$ in 1785 indicated, by way of systematic clinical investigation, that digitalis drugs have a diuretic and pulse slowing effect, these drugs have gained a widespread use in patients, who suffer from what we now know to be pump failure of the heart. It is also known for a long time that overdosing the drug frequently leads to toxic side effects.

This was shown first by Salerne in $1748^{104}$, who overfed turkeys with digitalis leaves. Digitalis intoxication leads frequently to the occurrence of cardiac arrhythmias. In the isolated tissue preparation triggered activity has been found to be an important mechanism for digitalis induced arrhy thmias. When triggered activity also plays a role in arrhythmias induced by digitalis in the intact heart, this has important diagnostic and therapeutic consequences.

Therefore, the aim of this study was to determine the role of triggered activity in the digitalis intoxicated intact heart.

Chapter 1 reviews briefly the clinical problem of digitalis-induced cardiac arrhythmias and discusses new insights in cellular mechanisms underlying these arrhythmias. Chapter 2 summarizes the most important mechanisms involved in normal and abnormal impulse formation in cardiac tissues, and the electrophysiologic basis for the present study.

Chapter 3 reports on the effect of different modes of pacing on interval and configuration of the first postpacing QRS-complex during digitalis induced ventricular tachycardia. It was found that electrical stimulation induced one or more QRS-complexes of a different morphology after which the spontaneously occurring tachycardia regained control. A direct linear relation was found between the interstimulus interval and the first postpacing QRS-interval. The interval was $150-200 \%$ of the duration of the interstimulus interval. This relation was largely independent from the number of stimuli given. Analysis of the QRS-configuration revealed that the first postpacing QRS-complex originated close to the site of stimulation. Comparison of the first postpacing QRS-morphology and the stimulated QRS-complexes, suggested that the former originated within the specialized conduction system.

Chapter 4 reports on further observations on the first postpacing QRS-interval during digitalis intoxication.

The effect of programmed electrical stimulation was determined during and after sustained ventricular tachycardia. During ventricular tachycardia similar results were obtained as described in chapter 3. After spontaneous termination of sustained ventricular tachycardia ectopic impulse formation could be reinduced by pacing. Interstimulus intervals of more than $400 \mathrm{~ms}$ induced a first postpacing interval equal to the interstimulus interval, whereas shorter interstimulus intervals intervals led to a first postpacing interval 
being twice this interval duration.

When toxicity had almost subsided, ectopic activity could only be induced following short interstimulus intervals (200-320 $\mathrm{ms})$ and the interval duration was again twice the interstimulus intervall. It is concluded that, at different levels of digitalis intoxication, triggered activity is the most likely mechanism leading to the first postpacing QRScomplex.

Chapter 5 deals with the morphology of the first postpacing QRS-complex during digitalis-induced ventricular tachycardia. It was found that the first postpacing QRSconfiguration is determined by the configuration of the spontaneous ventricular tachycardia and of the stimulated QRS-complexes. The faster and longer the stimulation train the more similar the first postpacing QRS-configuration became to the paced QRS-configuration. Changing only the last paced interval had a great influence on this behaviour.

These findings are explained by simultaneous induction of triggered activity at the site of stimulation and at the site of impulse formation of the sustained ventricular tachycardia. In this chapter also the group beating behaviour of a spontaneous tachycardia is explained by triggered activity, through analysis of QRS-configuration and interval following programmed electrical stimulation.

Chapter 6 reports on the effect of programmed electrical stimulation on the ventricular rhythm without digitalis intoxication. It was found that overdrive stimulation not only resulted in pacing rate dependent overdrive suppression, but also in a group of first postpacing QRS-complexes which behaved differently to programmed electrical stimulation.

These latter QRS-complexes were termed "premature escape beats". Premature escape beats: 1 ) were readily induced by short and fast drives ( $98 \%$ following pacing with 10 stimuli and $200 \mathrm{~ms}$ interstimulus intervals);2) had an interval duration which shortened on increasing the pacing rate; 3 ) had an interval duration which was not influenced by increasing the number of stimuli untill suppression occurred at a critical number of stimuli; 4) were followed by a longer (overdrive suppressed) second postpacing interval; 5) could also be induced by single extrastimuli following regular pacing. It is suggested that triggered activity is the underlying mechanism for premature escape beats.

To confirm this hypothesis the behaviour of premature escape beats was investigated in combination with drugs suppressing triggered activity (lidocaïne, verapamil), and inducing it (isoprenaline, ouabain). It was found that lidocaine and verapamil decreased the number of premature escape beats and lengthened their interval duration, whereas isoprenaline and ouabain had the opposite effect.

Higher levels of ouabain increased the number of premature escape beats also in the subsequent intervals following stimulation and decreased their interval duration until sustained ventricular tachycardia occurred.

It is concluded that premature escape beats are based on triggered activity. This means that triggered activity can also be induced by electrical stimulation in the absence of digitalis intoxication. Under these circumstances this mechanism of impulse formation is only observed in the first postpacing QRS-complex because simultaneously overdrive suppression is induced. Because digitalis inhibits the sodium-potassium pump (which is the most important mechanism for overdrive suppression), during digitalis intoxication triggered activity can persist in the subsequent postpacing intervals. 


\section{Samenvatting}

\section{Impulsvorming in de hartkamers en de invloed van digitalis intoxicatie}

Dit proefschrift gaat over ritmestoornissen van het hart, die veroorzaakt worden door digitalis intoxicatie.

Het vingerhoedskruid was al voor onze jaartelling bekend om zijn geneeskrachtige werking. In 1542 verkreeg het de wetenschappelijke benaming "digitalis" van de botanist en arts Leonhard Fuchs ${ }^{39}$.

Sinds Withering ${ }^{158}$ in 1785 door systematisch klinisch onderzoek aangaf dat digitalis preparaten een diuretische en polsvertragende werking hebben zijn deze middelen voorkeurspreparaten geweest bij patienten waarvan wij nu weten dat zij een verminderde pompfunctie van het hart hebben. Het is echter ook al lang bekend dat overdosering snel leidt tot toxische effecten. Dit is voor het eerst experimenteel aangetoond door Salerne in $1748^{104}$. Hij verrichtte dit onderzoek bij kalkoenen.

Een van de uitingen van digitalis intoxicatie is het optreden van ritmestoornissen van het hart. Wanneer een patient een ritmestoornis vertoont en tevens een digitalis preparaat gebruikt wordt vaak de diagnose digitalis intoxicatie overwogen. Ondanks de klinische verschijnselen, het electrocardiogram en de bepaling van plasmaspiegels van het digitalispreparaat, kan het moeilijk zijn deze diagnose met zekerheid te stellen.

Door onderzoek van gë̈soleerde hartweefselpreparaten is de laatste decennia veel inzicht ontstaan in de cellulaire mechanismen die aan deze ritmestoornissen ten grondslag liggen. Een van de belangrijkste mechanismen dat hierbij naar voren kwam is triggered activity. Ten gevolge van triggered activity wordt abnormale impulsvorming opgewekt door het tevoren aanwezig hartritme. Tijdens digitalis intoxicatie blijkt na beëindiging van het hartritme de membraanpotentiaal oscillaties te vertonen (delayed afterdepolarizations) (hoofdstuk 2, figuur 7A). Wanneer de amplitudo van deze nadepolarisaties voldoende hoog is, kan de prikkeldrempel worden overschreden en een volledige depolarisatie optreden (hoofdstuk 2, figuur 5). Deze wordt ook gevolgd door een nadepolarisatic die opnieuw aanleiding kan zijn tot een volledige depolarisatie enzovoorts.

Wanneer triggered activity ook in het intacte hart een rol speelt bij ritmestoornissen ten gevolge van digitalis intoxicatie, heeft dit belangrijke diagnostische en therapeurische consequenties.

Dit onderzoek was er dan ook op gericht om na te gaan of triggered activity ook aantoonbaar is in het intacte hart gedurende digitalis intoxicatie.

Hoofdstuk 1 geeft in het kort een overzicht van het klinisch probleem van digitalis intoxicatie, de ritmestoornissen die het gevolg ervan zijn en van mechanismen van ontstaan van deze ritmestoornissen.

Hoofdstuk 2 geeft een samenvatting van de literatuur betreffende de belangrijkste mechanismen die aan normale en abnormale impulsworming ten grondslag liggen. In hoofdstuk 3 wordt verslag gedaan van het effect van geprogrammeerde electrische 
stimulatie op het eerste QRS-interval en vorm tijdens door digoxine geinduceerde kamertachycardieën. Het resultaat van dit onderzoek was dat na stimulatie van het hart een kortdurende nieuwe tachycardie ontstaat, die zijn oorsprong vindt dichter bij de plaats van stimulatie dan het spontane ritme. Er was een rechtstreekse relatie tussen her interstimulus interval en het eerste QRS-interval na stimulatie. De duur van het eerste interval na stimulatie was $150-200 \%$ van het interstimulus interval. Deze relatie was grotendeels onafhankeliik van het aantal stimuli.

Analyse van het QRS-complex ten gevolge van stimulatie en het eerste QRS-complex na stimulatie suggereert dat het laatste ontstaat in het specifieke geleidingssysteem van de ventrikels. Deze resultaten passen bii de bevindingen gedaan bij geïsoleerde Purkinievezels.

In hoofdstuk 4 wordt verslag gedaan van het effect van geprogrammeerde stimulatie op het eerste eropvolgende QRS-interval, zowel tijdens als na spontane beëindiging van ventriculaire tachycardie. Gedurende ventriculaire tachycardieën worden de bevindingen uit het vorige hoofdstuk bevestigd. Na beëindiging van ventriculaire tachycardie werd ectopische impulsworming opnieuw opgewekt door geprogrammeerde stimulatie. $\mathrm{Na}$ drijwen met een interstimulus interval van meer dan $400 \mathrm{~ms}$ was het eerste QRS-interval gelijk aan het interstimulus interval terwijl stimuleren met een korter interval een tweemaal zo lang eerste QRS-interval na stimulatie veroorzaakte.

In de episode dat digitalis in toxicatie bijna verdwenen was kon ectopische impulsvorming alleen kon worden opgewekt door stimulatie met een kort interval (200-320 ms). Ook nu had het koppelingsinterval van het eerste QRS-complex na stimulatie een 2:l relatie met het interstimulus interval.

Deze bevindingen wijzen erop dat bij verschillende mate van digitalis intoxicatie triggered activity ten grondslag ligt aan het eerste QRS-complex na stimulatie.

Hoofdstuk 5 behandelt de invloed van electrische stimulatie op de vorm van het eerste eropvolgende QRS-complex tijdens kamertachycardie. Uit dit onderzoek bleek dat deze vorm bepaald wordt door de vorm van het $Q R S$-complex van de spontane tachycardie en van de gestimuleerde QRS-complexen. Naarmate sneller en langduriger werd gestimuleerd, bleek het QRS-complex erna sterker te lijken op de gestimuleerde QRScomplexen. Het laatste stimulatie-interval alleen bleek hierin opnieuw van grote invloed te zijn. Deze bevindingen worden verklaard door triggered activity bij de plaats van stimulatie en bij de oorsprongsplaats van de spontane tachycardie.

In dit hoofdstuk wordt tenslotte op grond van analyse van QRS-vorm en interval na electrische stimulatie, een spontane tachycardie geanalyseerd en worden de spontane QRS-intervallen van deze tachycardie verklaard op grond wan triggered activity. In hoofdstuk 6 wordt mededeling gedaan van de resultaten van de controle experimenten. Geprogrammeerde stimulatie werd toegepast zonder de aanwezigheid van digitalis intoxicatie. Het resultat van dit onderzoek was dat toename van de stimulatiefrequentie niet alleen leidt tot een toenemende verlenging van het eerste QRS-interval erna (overdrive suppression) maar in een aantal gevallen ook tot verkorting van het QRSinterval zoais gezien werd tijdens digitalis intoxicatie. Deze latste QRS-complexen werden "premature escape beats" genoemd. Premature escape beats: 1)traden vaker op bij snel en kortdurend stimuleren (b.v. in $98 \%$ van de stimulatietreinen met 10 stimuli en een interstimulus interval van $200 \mathrm{~ms}$; 2) toonden een rechtstreekse relatie tussen het 
interstimulus interval en hun koppelingsinterval; 3) hadden een koppelingsinterval dar onafhankelijk was van het aantal stimuli, maar bij een kritisch aantal stimuli konden premature escape beats niet meer worden opgewekt; 4) werden gevolgd door een langer tweede QRS-interval; 5) konden worden geïnduceerd door een enkele extrastimulus na regelmatig stimuleren.

Gesuggereerd wordt dat triggered activity het onderliggende mechanisme is voor deze QRS-complexen.

Om dit verder te analyseren werden premature escape beats onderzocht in combinatie met farmaca die triggered activity tegengaan (lidocaine, verapamil) en met farmaca die triggered activity opwekken (isoprenaline, ouabain) (hoofdstuk 7). Het bleek dat bij honden bij wie na een gegeven stimulatieprotocol de betreffende QRS-complexen waren op te wekken, deze door lidocaïne en verapamil onderdrukt worden, terwijl ouabain en isoprenaline het tegenovergestelde effect hadden.

Ouabain bleek bij toenemende toxiciteit het aantal premature escape beats, ook in de volgende intervallen na electrische stimulatie, te verhogen en hun intervalduur te verkorten. Dit leidde bij toenemende toxiciteit tot aanhoudende tachycardie. Het resultaat van dit onderzoek is dat premature escape beats berusten op triggered activity. Triggered activity is dus ook op te wekken zonder de anwezigheid van digitalis intoxicatie. Het wordt echter alleen gezien bij het eerste QRS-complex na stimulatie omdat simultaan overdrive suppression wordt opgewekt. Digitalis remt de natriumkalium pomp die het belangrijkste mechanisme is voor overdrive suppression. Daarom resulteert electrische stimulatie tijdens digitalis intoxicatie ook in triggered activity in de volgende QRS-complexen. 



\section{References}

1. Allessie MA, Bonke FIM, Schopman FJG: Circus movement in rabbit atrial muscle as a mechanism of tachycardia. III. The "leading circle" concept; a new model of circus movement in cardiac tissue without the involvement of anatomic obstacle. Circ. Res. 1977; 41: 9-18.

2. Allessie MA, Bonke FIM: Direct demonstration of sinus node reentry in the rabbit heart. Circ. Res. 1979; 44: 557-569.

3. Allessie MA, Lammers WJEP, Bonke FIM, Hollen J: Experimental evaluation of Moe's multiple wavelet hypothesis of atrial fibrillation. In: Zipes DP, Jalife J (Eds.). Cardiac electrophysiology and arrhythmias. Grune and Stratton Inc. Orlando 1984; 265-275.

4. Allessie MA, Lammers WJEP, Bonke FIM, Hollen J: Intra-atrial reentry as a mechanism for atrial flutter induced by acetylcholine and rapid pacing in the dog. Circulation 1984; 70: 123-135.

5. Antzelevitch C, Jalife J, Moe GK: Characteristics of reflection as a mechanism of reentrant arrhythmias and its relationship to parasystole. Circulation 1980; 61: 182-191.

6. Becker DJ, Nonkin PM, Bennett ZD, Kinball SG, Steinberg MS, Wasserman F: Effect of isoproterenol in digitalis cardiotoxicity. Am. J. Cardiol. 1962; 10: 242-245.

7. Borasio PG, Vassalle $\mathbf{M}$ : Effects of norepinephrine on active $\mathbf{K}$ transport and automaticity in cardiac Purkinje fibers. In: Myocardial Biology, Recent Advances in Studies on Cardiac Structure and Metablolism. Dhalla NS (Ed.). Baltimore: University Park, Press 1974; 41-57.

8. Brachmann J, Scherlag BJ, Rosenstraukh LV, Lazzara R: Bradycardia dependent triggered activity: Relevance to drug induced multiform ventricular tachycardia. Circulation 1983; 68: 846-856.

9. Brugada $\mathbf{P}$, Wellens $\mathbf{H J J}$ : The role of triggered activity in clinical ventricular arrhythmias. P.A.C.E. 1984; 7: 260-271.

10. Brugada P, Wellens HJJ: Electrophysiology, mechanisms, diagnosis, and treatment of paroxysmal recurrent atrioventricular nodal reentrant tachycardia. In: Surawicz B, Reddy CR, Prystowsky EN (Eds.). Martinus Nijhoff Publishers, Boston 1984; 131-157.

11. Butler VP, Jr.: Digoxin: Immunologic approaches to measurement and reversal of toxicity. New Engl. J. Med. 1970; 283: 1150-1156. 
12. Castellanos A, Lemberg L, Centurion MJ, Berkovits BV: Concealed digitalis induced arrhythmias unmasked by electrical stimulation of the heart. Am. Heart J. $1967 ; 73: 484490$.

13. Castellanos A Jr., Ortiz JM, Pastis N, Castillo C: The electrocardiogram in patients with pacemakers. Progr. Cardiovasc. Dis. 1970; 13: 190-195.

14. Castellanos A Jr., Maytin O, Lemberg L, Castillo C: Unusual QRS-complexes produced by pacemaker stimuli. Am. Heart J. 1977; 77: 732-737.

15. Clausen $T$, Flatman JA: The effect of catecholamines on Na-K transport and membrane potential in rat soleus muscle. J. Physiol. 1977; 270: 383-414.

16. Coumel P, Cabrol C, Fabiato A, Gourgon R, Slama R: Tachycardia permanente par rhythme réciproque. Arch. Mal Coeur 1967; 60: 1830-1835.

17. Cranefield PF, Aronson RS: Initiation of sustained rhythmic activity by single propagated action potentials in canine Purkinje fibers exposed to sodium free solution or to ouabain. Circ. Res. 1974; 34: 477-484.

18. Cranefield PF: Action potentials, afterpotentials and arrhythmias. Circ. Res. 1977; $41: 415-423$.

19. Damato AN, Lau HS, Bobb GA: Digitalis induced bundle branch ventricular tachycardia studied by electrode catheter recordings of the specialized conducting tissue of the dog. Circ. Res. 1971; 28: 16-22.

20. Damiano BP, Rosen $M$ : Effects of pacing on triggered activity induced by early afterdepolarizations. Circulation 1984; 69: 1013-1025.

21. Dangman K, Hoffman BF: Effects of nifedipine on electrical activity of cardiac cells. Am. J. Cardiol. 1980; 46: 1059-1067.

22. Dangman KM, Hoffman BF: Studies on overdrive stimulation of canine cardiac Purkinje fibers: Maximal diastolic potential as a determinant of the response. J.A.C.C. $1983 ; 2: 1183-1190$.

23. Davis LD, Temte JV: Electrophysiological actions of lidocaïne on canine ventricular muscle and Purkinje fibers. Circ. Res. 1969; 24: 639-655.

24. Den Dulk K, Bertholet $M$, Brugada P, Bär FWHM, Richards D, Demoulin JC, Waleffe A, Bakels N, Lindemans FW, Bourgeois I, Kulbertus HE, Wellens HJJ: A versatile pacemaker system for termination of tachycardias. Am. J. Cardiol. 1983; 52: $731-738$. 
25. Den Dulk K, Bertholet M, Brugada P, Bär FWHM, Demoulin JC, Waleffe A, Bakels N, Lindemans FW, Bourgeois I, Kulbertus HE, Wellens HJJ: Clinical experience with implantable devices for control of tachyarrhythmias. P.A.C.E. $1984 ; 7: 548-556$.

26. Di Francesco D: A new interpretation of the pacemaker current in calf Purkinje fibers. J. Physiol. 1981; 314: 359-376.

27. Durrer D, Schoo L, Schuilenburg RM, Wellens HJJ: The role of premature beats in the initiation and termination of supraventricular tachycardia in the WolffParkinson-White syndrome. Circulation 1967; 34: 644-662.

28. Durrer D, Roos JP: Epicardial excitation of the ventricles in a patient with WolffParkinson-W/hite syndrome. Circulation 1967, 35: 15-21.

29. Eisner DA, Lederer WJ: A cellular basis for lidocaines anti-arrhythmic action. J. Physiol. 1979; 295: 25-26.

30. El-Sherif N, Smith A, Evans K: Canine ventricular arrhythmias in the late myocardial infarction period: 8. epicardial mapping of reentrant circuit. Circ. Res. $1981 ; 45: 255-265$.

31. Erly $\mathbf{D}$, Mendez $\mathbf{R}$ : The modification of digitalis intoxication by excluding adrenergic influences on the heart. J. Physiol. 1964; 144: 97-103.

32. Ferrier GR, Saunders JH, Mendez C: A cellular mechanism for the generation of ventricular arrhythmias by acetylstrophanthidin. Circ. Res. 1973; 32: 600-609.

33. Ferrier GR, Moe GI: Effect of calcium on acetylstrophanthidin induced transient depolarizations in canine Purkinje tissue. Circ. Res. 1973; 33: 508-515.

34. Ferrier GR: The effects on tension on acetylstrophantidin induced transient depolarizations and aftercontractions in canine myocardial and Purkinje tissue. Circ. Res. 1976; 38: 156-162.

35. Ferrier GR: Digitalis arrhythmias: role of oscillatory afterpotentials. Prog. Cardiovasc. Dis. 1977; 19:459-474.

36. Ferrier GR: Effects of transmembrane potential on oscillatory afterpotentials induced by acetylstrophantidine in canine ventricular tissues. J. Pharmacol. Exp. Ther. 1980; $215: 332-341$.

37. Frame LH, Hoffman BF: Mechanisms of tachycardia. In: Surawicz B, Reddy CP, Prystowsky EN (Eds.). Tachycardias. Martinus Nijhoff Publishers, Boston 1984; 2-35. 
38. Friedberg CK, Donoso E: Arrhythmias and conduction disturbances due to digitalis. Prog. Cardiovasc. Dis. 1959; 2: 408-413.

39. Fuchs L: De Historia stirpium. p 892. Deutsches Kreuterbuch. Basel 1543.

40. Glitsch HG: Characteristics of active sodium transport in intact cardiac cells. In: Levy, Vassalle (Eds.). Excitation and neural control of the heart. American Physiological Society, Bethesda Maryland 1982; 37-58.

41. Goldreyer BN, Damato AN: Sinoatrial node entrance block. Circulation 1971; 44: 789-792.

42. Gorgels APM, Beekman HDM, Brugada P, Dassen WRM, Richards DAB, Wellens HJJ: Extrastimulus related shortening of the first postpacing interval in digitalis induced ventricular tachycardia. J.A.C.C. 1983; 1 (3): 840-857.

\section{Gorgels APM, de Wit B, Beekman HDM, Dassen WRM, Wellens HJJ:} Triggered activity induced by pacing during digitalis intoxication. (Accepted for publication).

44. Gorgels APM, de Wit B, Beekman HDM, Dassen WRM, Wellens HJJ: Effect of different modes of stimulation on the morphology of the first QRS-complex following pacing during digitalis induced ventricular tachycardia. (Accepted for publication).

45. Greef K, Schadewaldt H: Introduction and remarks on the history of cardiac glycosides. In: Greeff $\mathrm{K}$ (Ed.). Cardiac Glycosides, part 1. Experimental pharmacology, Springer Verlag, Berlin, Heidelberg, New York 1981; 1-12.

46. Grosse Bockhoff F, Peters U: Clinical indications and choice of cardiac glycosides; clinical conditions influencing glycoside effects. In: Greeff $\mathrm{K}$ (Ed.). Cardiac glycosides, part II. Pharmacokinetics and clinical pharmacology, Springer Verlag Berlin, Heidelberg, New York 1981; 232-274.

47. Hagemeyer $\mathbf{F}$, Lown B: Effect of heart rate on electrically induced repetinive ventricular responses in the digitalized dog. Circ. Res. 1970; 27:333-344.

48. Hashimoto K, Moe GK: Transient depolarizations induced by acetylstrophanthidin in specialised tissue of $\mathrm{dog}$ atrium and ventricle. Circ. Res. $1973 ; 32: 618-624$.

49. Hauswirth $\mathbf{O}$, Noble $\mathbf{D}, \mathbf{T}$ sien $\mathbf{R W}$ : Adrenaline: mechanism of action on the pacemaker potential in cardiac Purkinje fibers. Science 1968; 162: 916-917.

50. Hewett KW, Rosen MR: Alpha and beta adrenergic interactions with ouabaininduced delayed afterdepolarizations. Pharmacol. Exp. Ther. 1984; 229: 188-192. 
51. Hiraoka M, Okamoto $\mathbf{Y}$, Sano $\mathbf{T}$ : Oscillatory afterpotentials in dog ventricular muscle fibers. Circ. Res. 1981; 48: 510-518.

52. Hoffman BF, Singer DM: Effects of digitalis on electrical activity on cardiac fibers. Progr. Cardiovasc. Dis. 1964; 7: 266-271.

53. Hoffman DK, Azarnoff DL: Absorption of orally given digoxin preparations. J. Am. Med. Assac. 1972; 222: 957-960.

54. Hoffman BF, Rosen MR: Cellular mechanisms for cardiac arrhythmias. Circ. Res. $1981 ; 49: 1-15$.

55. Hoffman BF: Disturbances of cardiac electrogenesis. In: Rosenbaum MB, EJizari MV (Eds.). Frontiers of cardiac electrophysiology, development in cardiovascular medicine. Martinus Nijhoff Publishers, Boston 1983; 1-12.

56. Hoffman BF, Cranefield PF: The physiological basis of cardiac arrhythmias. Am. J. Med. 1964; 7: 670-684.

57. Hogan PM, Wittenberg SM, Klocke FJ: Relationship of stimulation frequency to au tomaticity in the canine Purkinje fiber during ouabain administration. Circ. Res. $1973 ; 32: 377-383$.

58. Hogan PM, Spitzer KW: Verapamil induced increases of Purkinje fiber automaticity. Fed. Proc. 1975; 34: 375 (Abstr.).

59. Ilvento JP, Provet J, Danilo P, Rosen MR: Fast and slow idioventricular rhythms in the canine heart; a study of their mechanism using antiarrhythmic drugs and electrophysiologic testing. Am. J. Cardiol. 1982; 49: 1909-1916.

60. Imanishi S, Surawicz B: Automatic activity in depolarized guinea pig ventricular myocardium. Circ. Res. 1976; 39: 751-759.

61. Isenberg J: Cardiac Purkinje fibers: $\left(\mathrm{Ca}^{2+}\right)$ controls the potassium permeability via the conductance components $\mathrm{gK}_{1}$ and $\mathrm{gK}_{2}$. Pfluegers Arch. 1977; 371: 77-85.

62. Jalife J, Moe GK: Effect of electrotonic potentials on pacemaker activity of canine Purkinje fibers in relation to parasystole. Circ. Res. 1976; 36: 801-808.

63. Janse MJ, van Capelle FJL, Freud GE, Durrer D: Circus movement within the AV-node. Circ. Res. 1971; 28: 403-414.

64. Janse MJ, van Capelle FJL, Morsink H, Kleber AG, Wilhs-Schopman F, Cardinal R, Naumann D'Alnoncourt C, Durrer D: Flow of "injury" current and patterns of excitation during early ventricular arrliythmias in acute regional myocardial ischemia in isolated porcine and canine hearts; evidence for two different arrhythmogenic mechanisms. Circ. Res. 1980; 47: 151-165. 
65. Janse $\mathbf{M J}$, Plumb VJ, Henthorn RW: Observations on the mechanism of atrial flutter. In: Surawicz B, Reddy CP, Prystowsky EN(Eds.). Tachycardias. Martinus Nijhoff Publishers, Boston 1984; 213-229.

66. Jelliffe RW, Buell J, Kalaba R: Reduction in digitalis toxicity by computer-assisted glycoside close regimens. Ann. Int. Med. 1972; 77: 891-906.

67. Jordan JL, Yamaguchi J, Mandel WJ: Function and dysfunction of the sinus node: clinical studies in the evaluation of sinus node function. In: The sinus node, Bonke FIM; (Ed.). Martinus Nijhoff Medical Division 1978; 3-22.

68. Josephson ME, Horowitz LN, Farshidi A, Kastor JA: Recurrent sustained ventricular tachycardia: 1. Mechanisms. Circulation 1978; 57:431-439.

69. Josephson ME, Harken AH: Surgical therapy of arrhythmias. In: Rosen MK, Hoffman BF (Eds.). Cardiac Therapy. Martinus Nijhoff Publishers, Boston 1983; $337-385$.

70. Kass RS, Tsien RW, Weingart R: Ionic basis of transient inward current induced by strophantidin in cardiac Purkinje fibers. J. Physiol. 1978; 281: 209-226.

71. Kastor JA, Spear JF, Moore EN: Localization of ventricular irritability by epicardial mapping. Circulation, 1972; 45: 952-964.

72. Killip T, Yormak S, Ettinger E, Levitt B, Roberts B: Depression of ventricular automaticity by electrical stimulation. Pharmacologist 1966; 8: 203 (Abstr.).

73. Kimura S, Cameron JS, Kozlovsky PL, Bassett AL, Meyerburg RJ: Delayed afterdepolarizations and triggered activity induced in feline Purkinje fibers by alfa-a drenergic stimulation in the presence of elevated calcium levels. Circulation 1984; $70: 1074-1082$.

74. Klevans LR, Kelly RJ: Effect of autonomic neural blockade on verapamil-induced stuppression of the accelerated ventricular escape beat in ouabain-treated dogs. J. Pharmacol. Exp. Ther. 1978; 206: 259-267.

75. Krellenstein DJ, Pliam MB, Brooks McC, Vassalle $\mathbf{M}$ : On the mechanism of idioventricular pacemaker suppression by fast drive. Circ. Res. 1974; 35: 923-934.

76. Krellenstein DJ, Pliam MB, Brooks McC, Vassalle M: Factors affecting overdrive suppression of idlioventricular pacemakers and associated potassium shifts. J. Electrocardiol. (San Diego) 1978; 11:3-10.

77. Lange G: Action of driving stimuli from intrinsic and extrinsic sources on in situ cardiac pacemaker tissues. Circ. Res. 1965; 17: 449-459. 
78. Lederer WJ, Tsien RW: Transient inward current underlying arrhythmogenic effects of cardiogenic steroids in Purkinje fibers. J. Physiol. 1976; 263: 73-100.

79. Lindenbaum J, Mellow VP: Variation in biologic availability of digoxin from four preparations. New Engl. J. Med. 1971; 285: 1344-1347.

80. Lown B, Camnon RL, Rossi MA: Electrical stimulation and digitalis drugs: repetitive response in diastole. Proc. Soc. Exp. Biol. Med. 1967; 1.26: 628-701.

81. Lown B: Electrical stimulation to estimate the degree of digitalization. Am. J. Cardiol. 1968; 22: 251-259.

82. Mandel WJ, Hayakawa $H$, Danzig R, Marcus NS: Evaluation of sino-atrial node function in man by overdrive suppression. Circulation 1971;44:59-66.

83. Manninen V, Melin J, Hartel G: Serum digoxin concentrations during treatment with different preparations. Lancet 1971; II, 934-935.

84. Mason JW, Winkle RA: Electrical catheter arrhythmia induction in the selection and assessment of anti-arrhythmic drug therapy for recurrent ventricular tachycardia. Circulation 1978;58: 971-985.

85. Mason DT, Forester JM: Side effects and intoxication of cardiac glycosides: manifestations and treatment. in: Greeff $\mathrm{K}(\mathrm{Ed}$.$) . Cardiac glycosides, part II.$ Pharmacokinetics and clinical pharmacology. Springer Verlag Berlin, Heidelberg, New York 1981; 275-297.

86. Miura DS, Rosen MR: The effects of ouabain on the transmembrane potentials and intracellular potassium activity of canine cardiac Purkinje fibers. Circ. Res, 1978; 42: 333-338.

87. Moak JP, Rosen MR: Induction and termination of triggered activity by pacing in isolated canine Purkinje fibers. Circulation 1984;69: 149-162.

88. Moe GK: On the multiple wavelet hypothesis of atrial fibrillation. Arch. Int. Pharmacodyn. Ther. 1962; 140: 183-188.

89. Musso E, Vassalle $\mathbf{M}$ : The role of calcium in overdrive suppression of canine cardiac Purkinje fibers. Circ. Res. 1982; 51: 167-180.

90. Olgilvie RI, Ruedy J: An educational program in digitalis therapy. J.A.M.A. 1972; 222: $50-55$.

91. Oliver GC Jr., Parker BM, Brasfield OL: The measurement of digitoxin in human serum of radio-immunoassay. J. Clin. Invest. 1968; 47: 1035-1046. 
92. Pliam MB, Krellenstein DJ, Brooks McC, Vassalle $M$ : Norepinephrine, potassium and overdrive suppression. Basic Res. Cardiol. 1977; 72:34-45.

93. Polimeni $\mathbf{P}$, Vassalle $\mathbf{M}$ : On the mechanism of oubain toxicity in Purkinje and ventricular muscle fibers at rest and during activity. Am. J. Cardiol. 1971;27: $622-629$.

94. Reiser J, Anderson GJ: Preferential sensitivity of the left canine Purkinje system to cardiac glycosides. Circ. Res. 1981; 49: 1043-1054.

95. Reuter H: The dependence of slow inward current in Purkinje fibers on the extracellular calcium concentration. J. Physiol. (London) 1967; 192: 479-492.

96. Rosenbaum MB: Classification of ventricular extrasystoles according to form. J. Electrocardiogr. 1969; 2: 289-298.

97. Rosen MR, Gelband HB, Hoffman FB: Correlation between effects of ouabain on the canine electrocardiogram and transmembrane potentials of isolated Purkinje fibers. Circulation 1973; 47:65-72.

98. Rosen MR, Wit AL, Hoffman BF: Electrophysiology and pharmacology of cardiac arrhythmias. IV. Cardiac antiarrhythmic and toxic effects of digitalis. Am. Heart. J. 1975; 89:391-399.

99. Rosen MR, Danilo P: Effects of tetrodotoxin, lidocaine, verapamil and AHR-2666 on ouabain induced delayed afterdepolarizations in canine Purkinje fibers. Circ. Res. 1980; 46: 117-124.

100. Rosen MR: Cellular mechanisms of cardiac arrhythmias. In: Harrison DC (Ed.). Cardiac arrhythmias, a decade of progress. GK Hall Medical Publishers Boston, Massachusetts 1981;25-38.

101. Rosen MR, Reder RF: Does triggered activity have a role in the genesis of cardiac arrhythmias? Ann. Int. Med. 1981; 94: 794-801.

102. Rosen MR, Hoffman BF: Electrophysiologic determinants of normal cardiac rhythms and arrhythmias. In: Rosen MR, Hoffman BF (Eds.). Cardiac therapy. Martinus Nijhoff Publishers, Boston 1983; 1-19.

103. Rothberger CJ, Winterberg H: Ueber den Einfluss von Strophantin auf die Reizbildungsfähigkeit der automatische Zentren des herzens. Pfluegers Arch. 1913, 150: $217-261$.

104. Salerne F: Observation de Botanique. Hist. Acad. Roy. Sci. Paris, Annee 1748 (1752); 74-75. 
105. Saunders JH, Ferrier GR, Moe GK: Conduction block associated with transient depolarizations induced by acetyl-strophantidin in isolated canine Purkinje fibers. Circ. Res. 1973; 32: 610-617.

106. Segers $M$ : Le battement auto-entretenu du coeur. Arch. Int. Pharmacodyn. 1947; 75: 144-156.

107. Shaw TRD: Bioavailability of cardiac glycosides. In: Greeff K (Ed.). Cardiac glycosides, part II. Pharmacokinetics and clinical pharmacology. Springer Verlag; Berlin, Heidelberg, New York 1981;169-187.

108. Smith TW, Butler VP Jr., Haber E: Determination of therapeutic and toxic serum digoxin concentrations by radio-immunoassay. New Engl. J. Med. 1969; 281: $1212-1220$.

109. Smith TW, Curfman GD, Green LH: The use and misuse of digoxin blood levels. In: Hurst JW (Ed.). The Heart, update I Mc Graw-Hill, New York 1979; 75-82.

110. Smith TW, Braunwald E: The management of heart failure. In: Braunwald E (Ed.). Heart disease. WB Saunders Company, Philadelphia 1980; 509-570.

11. Smith TW, Antman EM, Friedman PL, Blatt CM, Marsh JD: Digitalis glycosides: mechanisms and manifestations of toxicity, part I, Progress in Cardiovasc. Dis. 1984; 26: 413-458.

112. Smith TW, Antman EM, Friedman PL, Blatt CM, Marsh JD: Digitalis glycosides: mechanisms and manifestations of toxicity, part II, Progress in Cardiovasc. Dis. 1984; 26: 495-540.

113. Smith TW, Antman EM, Friedman PL, Blatt CM, Marsh JD: Digitalis glycosides: mechanisms and manifestations of toxicity, part III, Progress in Cardiovasc. Dis. 1984; 27:21-56.

114. Steiner C, Kovalik TW: A simple technique for production of chronic complete heart block in dogs. J. Appl. Physiol. 1968; 25: 631-632.

115. Toda N: Barium-induced automaticity in relation to calcium ions and norepinephrine in the rabbit left atrium. Circ. Res. 1970; 27:45-57.

116. Trautwein W, Gottstein U, Dudel J: Der Aktionsstrom der Myokard faser in Sauerstoffmangel. Pfluegers Arch. 1954; 260: 40-60.

117. Tsien RW, Carpentier DO: Ionic mechanisms of pacemaker activity in cardiac Purkinje fibers. Fed. Proc. 1978; 37: 2127-2131. 
118. Vanagt EJ, Wellens $\mathbf{H J J}$ : The electrocardiogram in digitalis intoxication. In: Wellens HJJ, Kulbertus $M E$ (Eds.). What's new in electrocardiography? Martinus Nijhoff Publishers, The Hague 1981; 315-343.

119. Vassalle $\mathbf{M}$, Karis J, Hoffman BF: Toxic effects of ouabain on Purkinje fibers and ventricular muscle fibers. Am. J. Physiol. 1962; 203: 433-436.

120. Vassalle $\mathbf{M}$, Greenspan $\mathbf{K}$, Hoffman BF: Analysis of arrhythmias induced by ouabain in intact dogs. Circ. Res. 1963; 13: 132-148.

121. Vassalle $\mathbf{M}$ : Electrogenic suppression of automaticity in sheep and dog Purkinje fibers. Circ. Res. 1970; 27: 361-377.

122. Vassalle M, Barnabei O: Norepinephrine and potassium fluxes in cardiac Purkinje fibers. Pfluegers Arch. 1971; 322: 287-303.

123. Vassalle $\mathbf{M}$, Carpentier $\mathbf{R}$ : Overdrive excitation: Onset of activity following fast drive in cardiac Purkinje fibers exposed to norepinephrine. Pfluegers Arch. 1972; 332: 198-205.

124. Vassalle $\mathbf{M}$, Carpentier R, Chan PC: The effects of norepinephrine and cyclic AMP on $\left(\mathrm{Na}^{+}+\mathrm{K}^{+}\right.$)-activated ATPase. Physiologist 1972; 15: 293 (Abstr.).

125. Vassalle $\mathbf{M}$, Cummins $\mathbf{M}$, Castro $\mathbf{C}$, Stuckey JH: The relationship between overdrive suppression and overdrive excitation in ventricular pacemaker in dogs. Circ. Res. 1976; 38: 367-374.

126. Vassalle $\mathbf{M}$, Krellenstein DJ, Pliam MB, Brooks McC: Potassium-related humoral transmission of overdrive suppression. J. Mol. Cell. Cardiol. 1977; 9: 921-931.

127. Vassalle $\mathbf{M}$ : The relationships among cardiac pacemakers. Circ. Res. 1977; 41: 269-277.

128. Vassalle M, Knob RE, Cummins M, Lara GA, Castro C, Stuckey JH: An analysis of fast idioventricular rhythm in the dog. Circ. Res. 1977; 41:218-226.

129. Vassalle $\mathbf{M}$ : Cardiac automaticity and its control. In: Levy MN, Vassalle $M$ (Eds.). Excitation and neural control of the heart. American Physiological Society 1982; 59.77.

130. Vassalle $\boldsymbol{M}$ : Physiological basis of normal and abnormal automaticity. In: Rosenbaum MB, Elizari MV (Eds.). Frontiers of cardiac electrophysiology, Martinus Nijhoff Publishers 1983; 120-143. 
131. Vassalle $\mathbf{M}$ : Overdrive excitation: The onset of spontaneous activity following a fast drive. In Zipes DP, Jalife J (Eds.). Cardiac electrophysiology and arrhythmias. Grune and Stratton Inc., Orlando 1985; 97-107.

132. Vick RL: Suppression of latent cardiac pacemaker: relation to slow diastolic depolarization. Am. J. Physiol. 1969; 217: 451-457.

133. Vincenzi FF, West TC: Release of autonomic mediators in cardiac tissue by direct subthreshold electrical stimulation. J. Pharmacol. Exp. Ther. 1962; 141: 185-194.

134. Wald RW, Waxman MB: Pacing induced automaticity in sheep Purkinje fibers. Circ. Res. 1981; 48 : 531-538.

135. Wellens HJJ, Schuilenburg RM, Durrer D: Electrical stimulation of the heart in patients with ventricular tachycardia. Circulation 1972; 46: 216-226.

136. Wellens HJJ, Lie KI, Durrer D: Further abservations on ventricular tachycardia as studied by electrical stimulation of the heart. Chronic recurrent ventricular tachycardia and ventricular tachycardia during acute myocardial infarction. Circulation 1974; 49: 647-653.

137. Wellens HJJ, Durrer D: Effect of procainamide, quinidine and aimaline on the Wolff-Parkinson-White syndrome. Circulation 1974; 50: 114-120.

138. Wellens HJJ, Durrer D: The role of an accessory atrioventricular pathway in reciprocal tachycardia. Observations in patients with and without the WolffParkinson-White syndrome. Circulation 1975; 52: 58-69.

139. Wellens HJJ, Düren DR, Liem KL, Lie KI: Effect of digitalis in patients with paroxysmal atrioventricular nodal tachycardia. Circulation 1975; 52: 779-788.

140. Wellens HJJ: The electrocardiogram in digitalis intoxication. In: Yu PN, Goodwin JF (Eds.). Progress in Cardiology 5. Lea and Febiger, Philadelphia 1976; 271-290.

141. Wellens HJJ: The electrophysiologic properties of the accessory pathway in the Wolff-Parkinson-White syndrome. In: Wellens HJJ, Lie KI, Janse MJ (Eds.). The conduction system of the heart. Stenfert Kroese, Leiden 1976, 567-587.

142. Wellens HJJ, Düren DR, Lie KJ: Observations on mechanisms of ventricular tachycardia in man. Circulation 1976; 54: 237-244.

143. Wellens HJJ, Bär FWHM, Lie KI, Düren DR, Dohmen HJ: Effect of procainamide, propanolol, and verapamil on mechanism of tachycardia in patients with chronic recurrent ventricular tachycardia. Am. J. Cardiol. 1977; 40:579.585. 
144. Wellens HJJ, Bär FWHM, Gorgels APM, Farré Muncharaz J: Electrical management of arrhythmias with emphasis on the tachycardias. Am. J. Cardiol. $1978 ; 41: 1025-1034$.

145. Wellens HJJ: Role of sinus node reentry in the genesis of sustained cardiac arrlaythmias. In: Bonke FIM (Ed.). The sinus node. Martinus Nijhoff Medical Division, The Hague $1978 ; 422-427$.

146. Wellens HJJ: Value and limitations of programmed electrical stimulation of the heart in the study and treatment of tachycardia. Circulation 1978; 57: 845-853.

147. Wellens HJJ: Assessment and treatment of paroxysmal tachycardia. Hart Bulletin 1982; 13:51-58.

148. Wellens HJJ, Bär FWHM, Vanagt EJDM, Brugada P: Medical treatment of ventricular tachycardia: Considerations of the selection of patients for surgical treatment. Am. J. Cardiol. 1982; 49: 186-193.

149. Wit AL, Hoffman BF, Cranefield PF: Slow conduction and reentry in the ventricular conducting system. I. Return extrasystole in canine Purkinje fibers. Circ. Res. 1972; 30: 1-10.

150. Wit AL, Cranefield PF: Triggered activity in cardiac muscle fibers of the simian mitral valve. Circ. Res. 1976; 38: 85-98.

151. Wit AL, Cranefield PF: Triggered and automatic activity in the canine coronary sinus. Circ. Res. 1977; 44: 435-445.

152. Wit AL, Wiggins JR, Cranefield PF: Some effects of electrical stimulation on impulse initiation in cardiac fibers; its relevance for the determination of the mechanisms of clinical cardiac arrhythmias. In: Wellens HJJ, Lie KI, Janse MJ (Eds.). The conduction system of the heart Martinus Nijhoff Medical division, The Hague 1.978; 169-181.

153. Wit AL, Cranefield PF, Gadsby DC: Electrogenic sodium extrusion can stop triggered activity in the canine coronary sinus. Circ. Res. 1981; 49: 1029-1042.

154. Wit AL, Allessie MA, Fenoglio JJ, Bonke FIM, Lammers WJEP, Smeets J: Significance of the endocardial and epicardial border zones in the genesis of myocardial infarction arrhythmias. In: Harrison DC (Ed.). Cardiac arrhythmias, a decade of progress. GK Hall Medical Publishers, Boston, Massachusetts 1981, $39-68$.

155. Wit AL, Cranefield PF: Reentrant excitation as a cause of cardiac arrhythmias. In: Levy $M N$, Vassalle $M$ (Eds.). Excitation and neural control of the heart. American Physiological Society $1982 ; 113-148$. 
156. Wit AL, Allessie MA, Bonke FIM, Lammers W, Smeets J, Fenoglio JI Jr: Electrophysiological mapping to determine the mechanism of experimental ventricular tachycardia initiated by premature impulses: Experimental approach and initial results demonstrating reentrant excitation. Am. J. Cardiol. 1982;49: 166-185.

157. Wit AL, Rosen MK: Cellular electrophysiology of cardiac arrhythmias. In: Josephson ME, Wellens HJJ (Eds.). Tachycardias. Lea and Febiger, Philadelphia $1984 ; 1-27$.

158. Withering W: An account of the foxglove and some of its medical uses; with practical remarks on dropsy and other diseases. Birmingham 1785.

159. Wittenberg SM, Streuli F, Klocke FJ: Acceleration of ventricular pacemakers by transient increases in heart rate in dogs during ouabain administration. Circ. Res. 1970; 26: 705-716.

160. Wittenberg SM, Gandel P, Hogan PM, Kreuzer W, Klocke FJ: Relationship of heart rate to ventricular automaticity in dogs during ouabain administration. Circ. Res. 1972; 30: 167-176.

161. Zipes DP, Arbel E, Knope RF, Moe GK: Accelerated cardiac escape rhythms caused by ouabain intoxication. Am. J. Cardiol. 1974; 33: 248-253. 


\section{Slotwoord}

Velen wil ik danken, die hebben bijgedragen aan het tot stand komen van dit proefschrift. Professor Hein Wellens leidde mij op tot cardioloog; ik heb grote waardering voor zijn wetenschappelijke en op de patient gerichte benadering van de cardiologie. Hij stimuleerde mij dit onderzoek te beginnen en gaf mij de gelegenheid het te voltooien, ondanks de drukte die eern zich snel ontwikkelende klinische afdeling met zich mee brengt.

Professor Michael Rosen, your expert knowledge of basic electrophysiology and detailed comments to the manuscript have been of great help to me in the preparation of this thesis. Professor Maurits Allessie, je belangstelling voor het onderzoek, de vele uren die je besteedde aan het bespreken van de resultaten en het gedetailleerde commentaar op het manuscript zijn voor mij van grote betekenis geweest.

Professor Karl Heinz Rahn en Pedro Brugada wil ik danken voor het beoordelen van het manuscript en het waardevolle commentaar .

Jet Leunissen-Beekman is door haar onvolprezen inzet en kwaliteiten absoluut onmisbaar geweest bij het tot stand komen van dit proefschrift.

Bram de Wit heeft mij door zijn kennis en belangstelling zeer gestimuleerd. Zijn inbreng is onontbeerlijk geweest voor het resultaat van de controle experimenten.

Pim Dassen heeft mii door zijn coördinatie en adviezen bij het opstarten van het laboratorium, de ontwikkeling van de apparatuur en de voorbereiding van het proefschrift in vele opzichten geholpen.

Lenny Frissen-Cruts heeft met accuratesse en voortvarendheid de secretariële aspecten van het proefschrift ter hand genomen, samen met de overige medewerksters van het secretariële team van de capaciteitsgroep en met Emmy van Roosmalen-Huynen. Ton van den Bogaard, Frans Slangen en Huub Simons droegen zorg voor de proefdieren. Ruud Kruger en zijn medewerkers verleenden biotechnische ondersteuning. Bert van der Steld verzorgde met zijn medewerkers de electrotechniek en ontwikkelde met Guy Maris nauwkeurige apparatuur voor QRS-complex herkenning en interval meting, in combinatie met een computer gestuurde programmeerbare electrische stimulator.

Jo Houben en Annie Giebels vervaardigden de fotografische illustraties. Chris Voskamp verzorgde de lay-out en de omslag. Gerrie Sciarone-Gorgels spoorde de oorspronkelijke publicatie van Salerne op in de Bibliotheque Nationale in Parijs.

Miep Kleijne, Leon van Loo en Joost Drenth waren behulpzaam bij de analyse van de onderzoekgegevens.

Miel Cheriex, Ruud Krijne en Jeroen Hameleers assisteerden bij de uitvoering van een aantal experimenten.

Medtronic Company, Kerkrade, vervaardigde de electrodecatheters.

Tot slot een woord van dank aan mijn ouders, aan Janneke, Tönnes, Christoffel en Hannes, aan wie ik dit proefschrift opdraag. 


\section{Curriculum vitae.}

Antonius Peter Maria Gorgels was borm on March 2, 1948 in Kerkrade, The Netherlands. He attended gymnasium B at Henric van Veldeke College in Maastricht, where he graduated in 1965. He went to medical school in Nijmegen and graduated in February 1973. From March 1973 to April 1974 he served Her Majesty the Queen Juliana of The Netherlands as a medical officer. In this function he was trained at the Anesthesiology Department of the Radboud hospital in Nijmegen (head Prof.Dr. J.F. Crul). In April 1974 he started his residency in Internal Medicine at the department of Internal Medicine of the St. Canisius hospital in Nijmegen (head Dr. J.H.J. Enneking). From September 1976 he was resident at the department of Internal Medicine of the Radboud Hospital in Nijmegen (head Prof.Dr. C.L.H. Majoor). During this episode he attended the pulmonology department of the Medical Centre Dekkerswald in Groesbeek (head Prof. C.M. Jongerius)

In February 1977 his cardiology training started at the department of Cardiology of the St. Annadal Hospital in Maastricht (head Prof. Dr. H. J.J. Wellens). In October 1980 he was registered as a cardiologist and became a member of the capaciteitsgroep of Cardiology of the University of Limburg. 